\title{
WestVirginiaUniversity
}

THE RESEARCH REPOSITORY @ WVU

Graduate Theses, Dissertations, and Problem Reports

2009

\section{Effect of strain on aluminum nitride/gallium nitride Distributed Bragg Reflectors}

Christopher Michael Miller

West Virginia University

Follow this and additional works at: https://researchrepository.wvu.edu/etd

\section{Recommended Citation}

Miller, Christopher Michael, "Effect of strain on aluminum nitride/gallium nitride Distributed Bragg Reflectors" (2009). Graduate Theses, Dissertations, and Problem Reports. 4503.

https://researchrepository.wvu.edu/etd/4503

This Thesis is protected by copyright and/or related rights. It has been brought to you by the The Research Repository @ WVU with permission from the rights-holder(s). You are free to use this Thesis in any way that is permitted by the copyright and related rights legislation that applies to your use. For other uses you must obtain permission from the rights-holder(s) directly, unless additional rights are indicated by a Creative Commons license in the record and/ or on the work itself. This Thesis has been accepted for inclusion in WVU Graduate Theses, Dissertations, and Problem Reports collection by an authorized administrator of The Research Repository @ WVU. For more information, please contact researchrepository@mail.wvu.edu. 


\title{
EFFECT OF STRAIN ON ALUMINUM NITRIDE/GALLIUM NITRIDE DISTRIBUTED BRAGG REFLECTORS
}

\author{
by
}

Christopher Michael Miller

Thesis submitted to the College of Engineering and Mineral Resources at West Virginia University in partial fulfillment of the requirements

for the degree of

\author{
Master of Science \\ in \\ Electrical Engineering
}

Approved by

Dimitris Korakakis, Ph.D., Committee Chairperson

Lawrence A. Hornak, Ph.D.

Xian-An Cao, Ph.D.

Lane Department of Computer Science and Electrical Engineering

Morgantown, West Virginia 2009

Keywords: Aluminum Nitride, Gallium Nitride, Distributed Bragg Reflectors

Copyright 2009 Christopher M. Miller 


\section{ABSTRACT \\ EFFECT OF STRAIN ON ALUMINUM NITRIDE/GALLIUM NITRIDE DISTRIBUTED BRAGG REFLECTORS}

\section{by Christopher M. Miller}

III - N Distributed Bragg Reflectors (DBRs) are important components to nitride based optoelectronic devices. Nitride based DBRs are critical to the operation and performance of both Vertical Cavity Surface Emitting Lasers (VCSELs) and Light Emitting Diodes (LEDs). Aluminum Nitride (AlN)/Gallium Nitride DBRs are of particular interest because their high refractive index contrast allows the fabrication of DBRs with high reflectivity using a relatively low number of periods.

The growth of high quality AlN/GaN DBRs has been held back because of tensile strain resulting from the $2.4 \%$ lattice mismatch between $\mathrm{AlN}$ and GaN. This tensile strain has led to cracking in DBRs and has significantly decreased their overall reflectivity. Reducing this strain and subsequent cracking is critical to improving AlN/GaN DBR reflectivity and the overall efficiency of nitride based optoelectronic devices.

This work will focus on the characterization, simulation, and development of AlN/GaN DBRs. Individual AlN and GaN thin films will be characterized to determine their properties and promise for development DBR structures. AlN/GaN DBR structures will then be characterized and simulated to determine their overall performance. Finally, the effect of strain on both the apparent refractive index of individual layers and the overall performance of AlN/GaN DBRs will be investigated. 


\section{ACKNOWLEDGMENTS}

I would like to first thank my parents, Joseph and Phyllis Miller, whose hard work and support made my collegiate career possible. I would like to thank my advisor and committee chair, Dr. Dimitris Korakakis, for his encouragement, guidance, and funding throughout my entire graduate and much of my undergraduate career. I would like to thank my committee members, Dr. Lawrence A. Hornak and Dr. Xian-An Cao, for all the time and support they have contributed to this work. I would like to thank Dr. Kolin Brown for his help in learning and maintaining lab equipment. Finally, I would like to thank Lee Rodak, Ronak Rahimi, Srikanth Raghavan, John Harman, Joshua Nightingale, Rich Farrell, Adam Kabulski, Vincent Pagán, and my other coworkers for their support of this work as well as other projects I have worked on during my undergraduate and graduate studies. 


\section{TABLE OF CONTENTS}

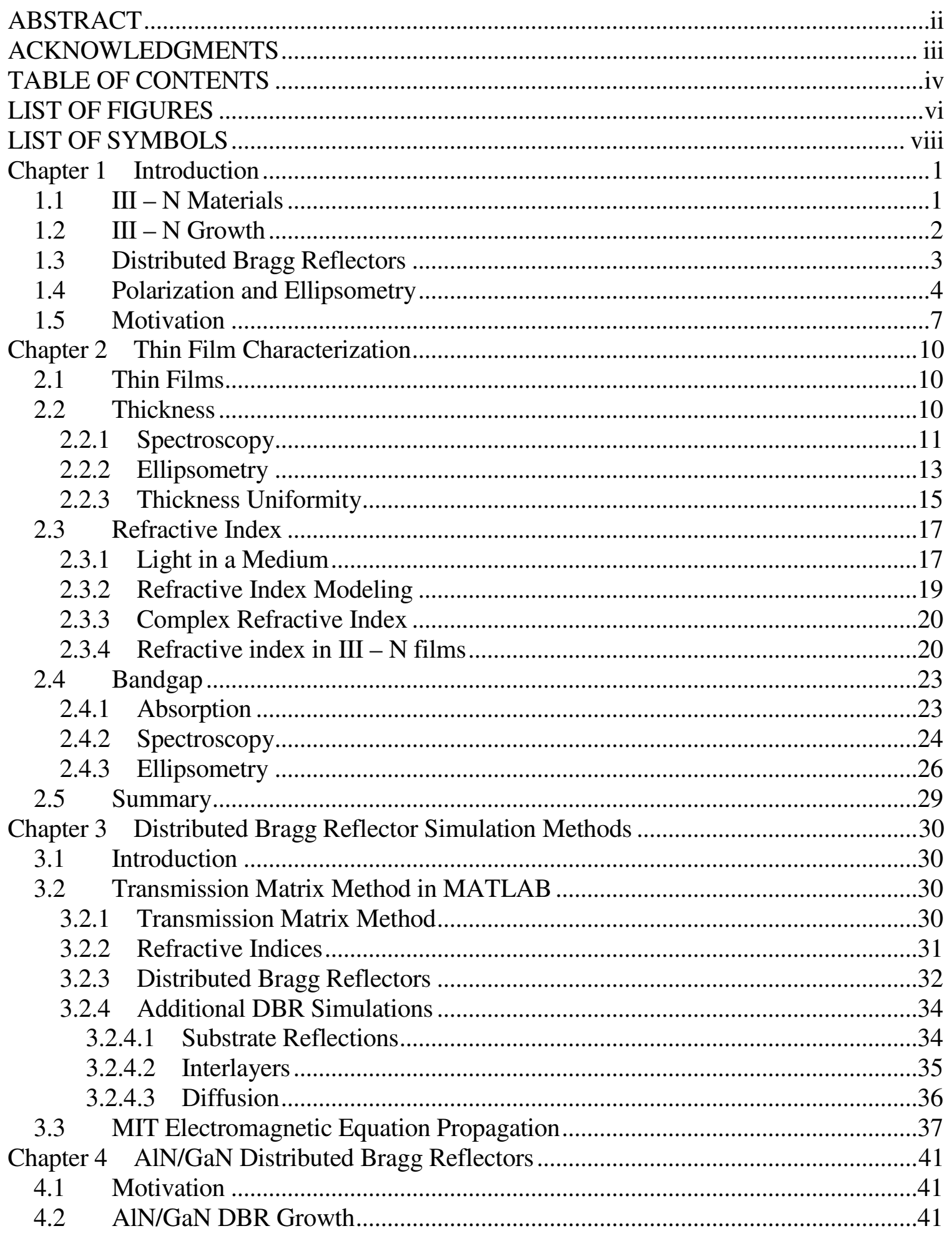




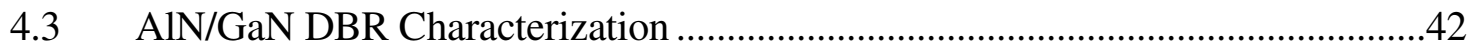

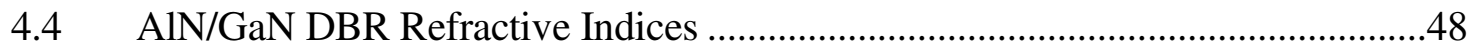

Chapter 5 Effect of Strain on AlN/GaN Distributed Bragg Reflectors ............................52

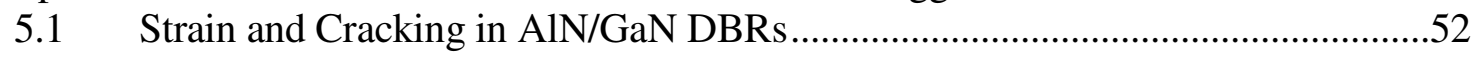

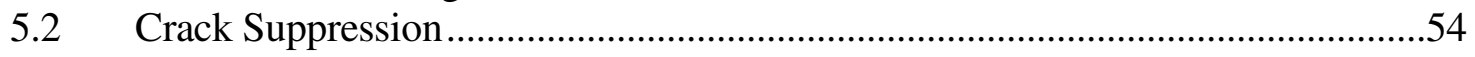

5.3 Effect of Strain on Refractive Index and DBR Reflectivity ...............................56

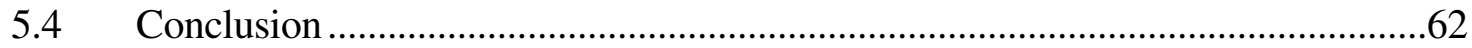

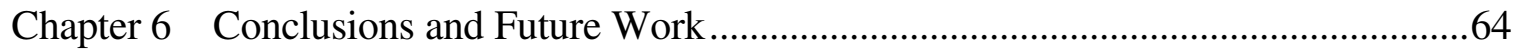

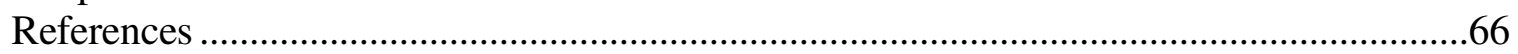

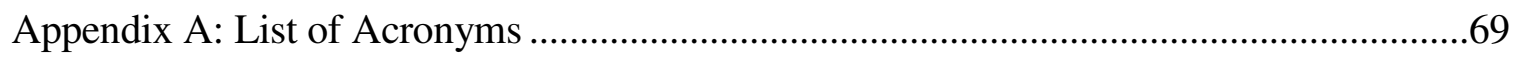

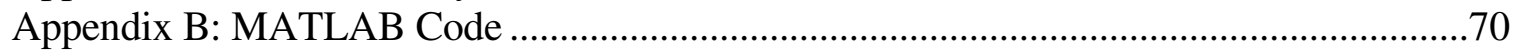

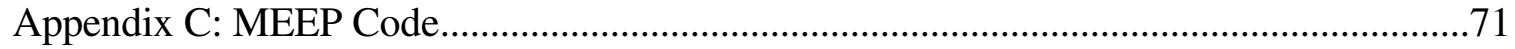

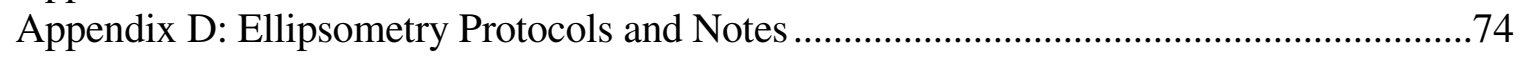




\section{LIST OF FIGURES}

Figure 1.1.1 III - N bandgaps vs. lattice constants [34] ...................................................2

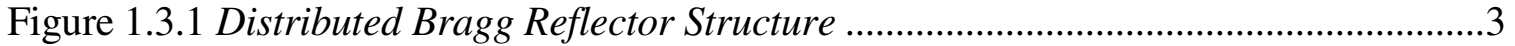

Figure 1.4.1 Polarization States Utilized by Ellipsometry [13] ............................................5

Figure 1.4.2 Flow Chart of Ellipsometry [14] ....................................................................

Figure 1.5.1 III - N LED Structure [16] .........................................................................

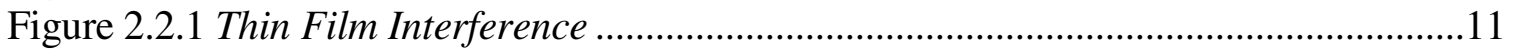

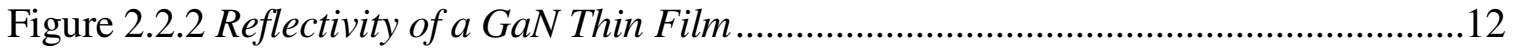

Figure 2.2.3 Linear Regression of Interference Fringes ...................................................13

Figure 2.2.4 Sample Ellipsometry Model ........................................................................14

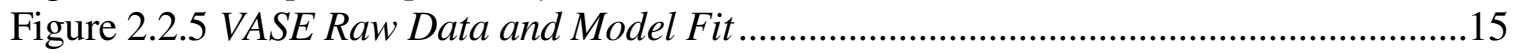

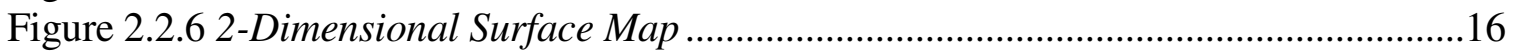

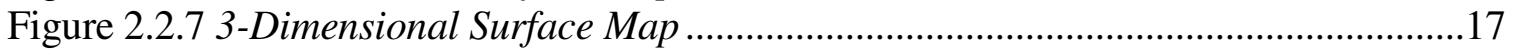

Figure 2.3.1 Snell's Law [22] .......................................................................................19

Figure 2.3.2 Refractive Index of a MOVPE Grown GaN Thin Film ...................................21

Figure 2.3.3 Extinction Coefficients of MOVPE Grown GaN Thin Films ...........................22

Figure 2.4.1 Allowed Direct Optical Transitions [23] ....................................................23

Figure 2.4.2 Square of Absorption Coefficient vs. Photon Energy for a GaN Film .............25

Figure 2.4.3 Square of Absorption Coefficient vs. Photon Energy for InGaN Films ...........26

Figure 2.4.4 Refractive Index Raw Data for an AlGaN Film ...........................................27

Figure 2.4.5 Peak Location vs. Aluminum Concentration ...................................................28

Figure 2.4.6 Comparison of Bandgaps vs. Aluminum Concentration in AlGaN films .........29

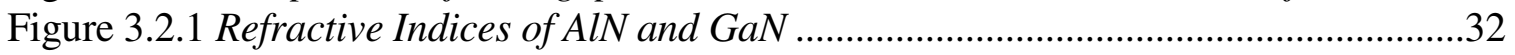

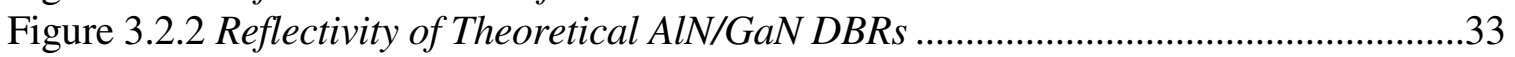

Figure 3.2.3 Refractive Index Contrast between AlN and GaN ..........................................33

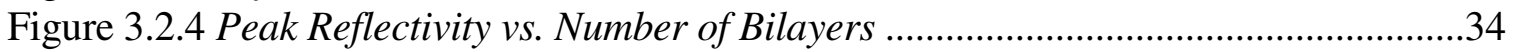

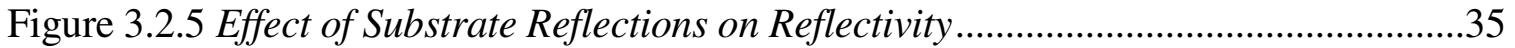

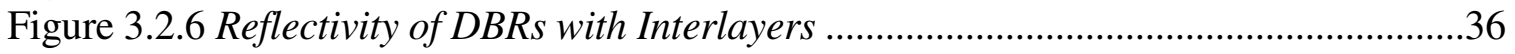

Figure 3.2.7 Effect of Diffusion on DBR Reflectivity ..........................................................

Figure 3.3.1 MEEP Output (DBR Structure and Field Pattern) .........................................39

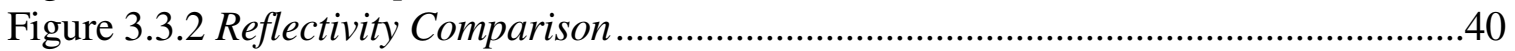

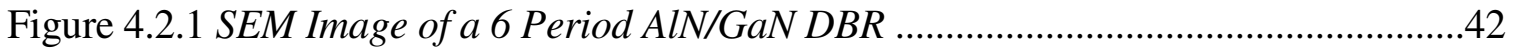

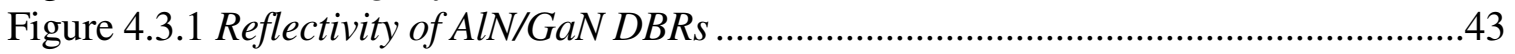

Figure 4.3.2 DBR Peak Reflectivity Compared to Theoretical ............................................44

Figure 4.3.3 Normalized Refractive Indices for a 540nm DBR .........................................45

Figure 4.3.4 Normalized GaN Thicknesses for a 540nm DBR .........................................46

Figure 4.3.5 Normalized AlN Thicknesses for a 540nm DBR ............................................46

Figure 4.3.6 Comparison of Simulated and Measured Reflectivity for a 540nm DBR .........47

Figure 4.3.7 Comparison of Simulated and Measured Reflectivity for a 515nm DBR ........48

Figure 4.4.1 DBR Refractive Indices Compared to Literature ............................................49

Figure 4.4.2 GaN Refractive Index Comparison..............................................................50

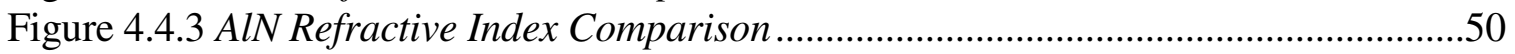


Figure 4.4.4 Refractive Index Contrast for DBRs and Literature ......................................51

Figure 5.1.1 Optical Microscope Image of a 6 Period AlN/GaN DBR .................................53

Figure 5.1.2 Reflectivity of AlN/GaN DBR Compared to Theoretical ....................................54

Figure 5.2.1 Effect of TMIn/TMAl ratio on Domain Density [37] .......................................55

Figure 5.3.1 Average GaN Refractive Index at 500nm for 6-Period DBRs .........................56

Figure 5.3.2 Average AlN Refractive Index at 500nm for 6-Period DBRs ..........................57

Figure 5.3.3 GaN Refractive Index at 500nm vs. Domain Density ......................................58

Figure 5.3.4 AlN Refractive Index at 500nm vs. Domain Density ........................................58

Figure 5.3.5 Average GaN Refractive Index at 500nm for AlN/GaN DBRs ........................59

Figure 5.3.6 Reflectivity of 460nm DBRs with Varying Domain Densities .........................60

Figure 5.3.7 Reflectivity of 540nm DBRs with Varying Domain Densities .........................61

Figure 5.3.8 Reflectivity of 486nm DBRs with Varying Domain Densities .........................61

Figure 5.3.9 Peak Reflectivity vs. Domain Density for DBRs ..........................................62 


\section{LIST OF SYMBOLS}

$\begin{array}{ll}\mathrm{c} & : \text { Speed of Light in Vacuum } \\ \mathrm{B} & : \text { Magnetic Field } \\ \mathrm{D} & : \text { Displacement Field } \\ \mathrm{E} & : \text { Electric Field } \\ \mathrm{E}_{\mathrm{g}} & : \text { Bandgap } \\ \mathrm{E}_{\lambda} & : \text { Frergy of Light at Wavelength ' } \lambda \text { ' } \\ \mathrm{f} & : \text { Magnetic Field Strength } \\ \mathrm{H} & : \text { Extinction Coefficient } \\ \mathrm{K} & : \text { Refractive Index } \\ \mathrm{n} & : \text { Reflectivity } \\ \mathrm{R} & : \text { Film Thickness } \\ \mathrm{t} & : \text { Speed of Light in a Medium with Refractive Index 'n' } \\ \mathrm{V}_{\mathrm{n}} & : \text { Optical Impedance } \\ \mathrm{Y} & : \text { Absorption Coefficient } \\ \alpha & : \text { Ellipsometry Phase Component } \\ \Delta & : \text { Permittivity } \\ \varepsilon & : \text { Wavelength } \\ \lambda & : \text { Permeability } \\ \mu & : \text { Frequency of Light } \\ v & : \text { Free Charges } \\ \rho & : \text { Ellipsometry Amplitude Component } \\ \Psi & \end{array}$




\section{Chapter 1 Introduction}

\subsection{III - N Materials}

Group III - N Semiconductors; specifically Aluminum Nitride (AIN), Indium Nitride (InN), and Gallium Nitride $(\mathrm{GaN})$, are of considerable interest due to their physical properties and applications. These materials are well suited for high temperature and high power applications ranging from electric vehicles and avionics to power distribution [1]. These materials are also ideal for optoelectronic devices such as laser diodes, LEDs, and photodetectors ranging from IR to UV. Their unique properties have made them a primary material system for short wavelength optoelectronic devices [2].

III - $\mathrm{N}$ materials have direct bandgaps, meaning an electron can fall from the conduction band to the valence band without undergoing a momentum change, allowing it to give off the energy difference, equal to the bandgap, as a photon [3]. This ability makes direct bandgap semiconductors better suited for optoelectronic devices than indirect bandgap semiconductors which require an additional momentum change, accomplished through interaction with a phonon, or lattice vibration [4]. This interaction significantly decreases the efficiency of recombination and results in energy loss in the form of heat dissipation.

The bandgaps: $E_{g}=1.9 \mathrm{eV}$ for $\mathrm{InN}, 3.4 \mathrm{eV}$ for $\mathrm{GaN}$, and $6.2 \mathrm{eV}$ for AlN are wide ranging bandgaps that cover the spectrum from visible to ultraviolet [1,5]. Figure 1.1.1 shows the lattice constants and bandgaps for $\mathrm{AlN}, \mathrm{GaN}$, and $\mathrm{InN}$ and their ability to be tuned from $1.9 \mathrm{eV}$ to $6.2 \mathrm{eV}$ using ternary (AlGaN, InGaN) and quaternary (AlGaInN) alloying [34]. These materials have the advantage of having relatively close lattice constants, which allow ternary and quaternary alloys to be produced with minimal lattice

effects. This results in the bandgap being able to be tuned with less strain and higher film quality then other ternary compounds [6]. This versatility makes the III - N material system ideal for a wide range of optoelectronic and other applications. 


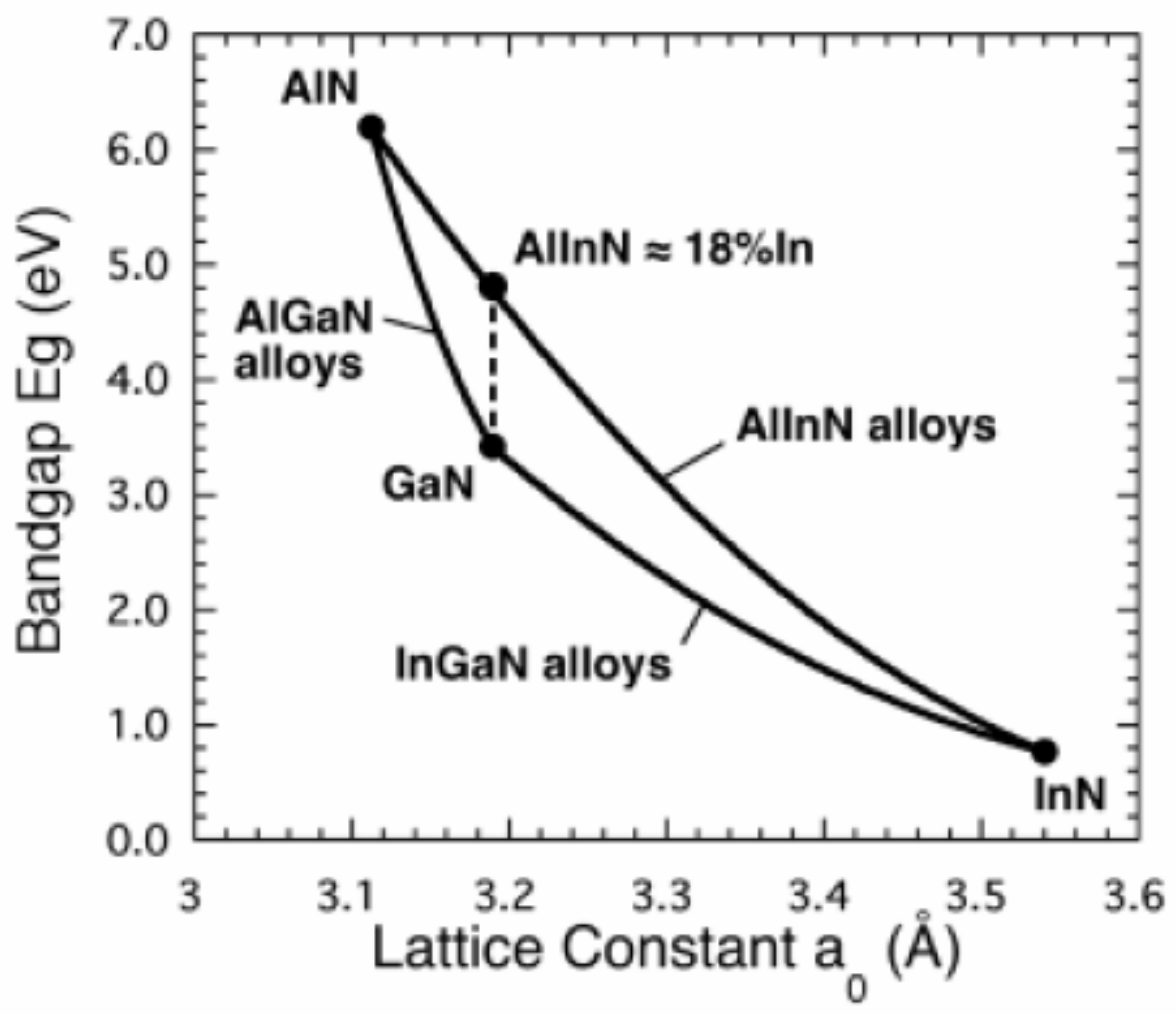

Figure 1.1.1 III - N bandgaps vs. lattice constants [34]

\subsection{III - N Growth}

While III - $\mathrm{N}$ materials are very useful for numerous applications, they are not commercially available in bulk and must be grown using epitaxial methods on foreign substrates such as sapphire, silicon, or silicon carbide [8]. Growing on these foreign substrates can result in high amount of strain and defect densities due to lattice mismatch [9]. For example, Gallium Nitride grown on sapphire has a 16\% lattice mismatch [10]. The methods for epitaxial growth include Metal Organic Vapor Phase Epitaxy (MOVPE), Molecular Beam Epitaxy (MBE), and Hydride Vapor Phase Epitaxy (HVPE). This work focuses on III - N materials grown on sapphire using MOVPE in an AIXTRON 200/4 RFS horizontal reactor. Trimethylgallium (TMGa), Trimethylaluminum (TMAl), 
Trimethylindium (TMIn), and ammonia (NH3) are used as the sources for Gallium, Aluminum, Indium, and Nitrogen, respectively.

\subsection{Distributed Bragg Reflectors}

Distributed Bragg Reflectors (DBRs) are periodic systems of quarter wavelength thick layers of alternating high and low refractive index materials [11]. DBRs are also known as quarter-wave stacks and Bragg Mirrors. DBRs are designed so that the thickness of each layer is related to the design wavelength of the reflector following equation 1.1:

$$
t=\frac{\lambda_{0}}{4 n}
$$

Where $\lambda_{0}$ is the design wavelength in vacuum and $\mathrm{n}$ is the refractive index of that layer.

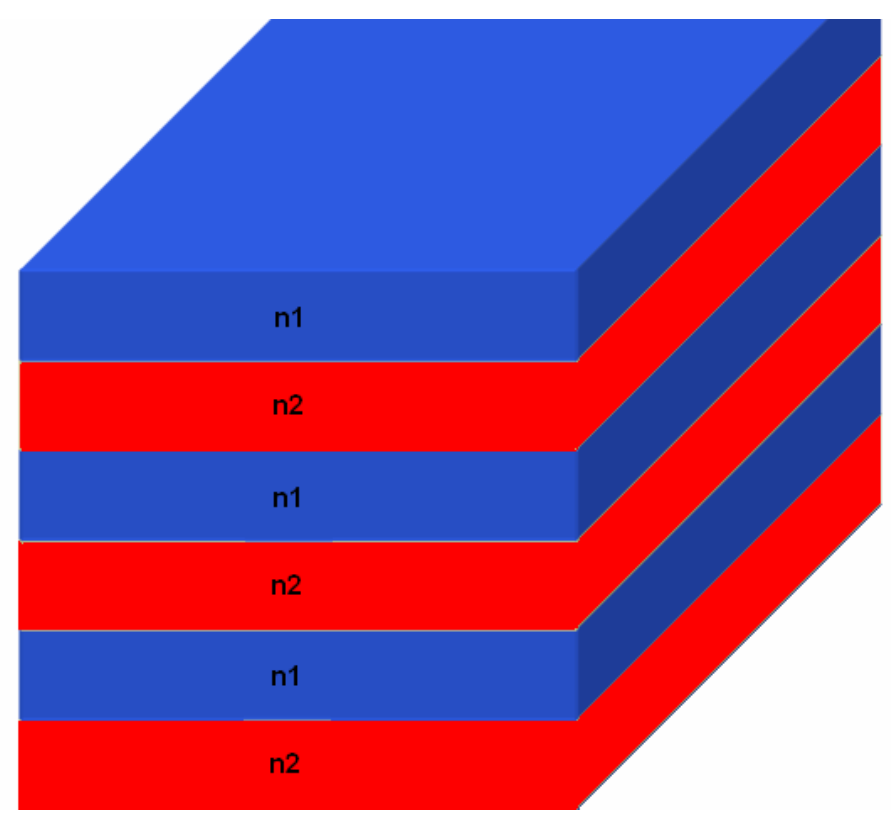

Figure 1.3.1 Distributed Bragg Reflector Structure

An example of a DBR is shown in figure 1.3.1. Light incident on the top of the structure will be reflected from each layer following Fresnel's equations as shown in 
equation 1.2 for light perpendicular to the plane of incidence and equation 1.3 for light parallel to the plane of incidence:

$$
\begin{aligned}
& r_{\perp}=\frac{n_{1} \cos \theta-n_{2} \cos \theta}{n_{1} \cos \theta+n_{2} \cos \theta} \\
& r_{l l}=\frac{n_{2} \cos \theta-n_{1} \cos \theta}{n_{1} \cos \theta+n_{2} \cos \theta}
\end{aligned}
$$

Simplified for normal incidence, both of these equations reduce to:

$$
r=r_{\perp}=r_{l l}=\frac{n_{1}-n_{2}}{n_{1}+n_{2}}
$$

When $\mathrm{n} 1>\mathrm{n} 2$, the equation is positive and the reflection undergoes no phase change. For $\mathrm{n} 1<\mathrm{n} 2$, the equation is negative and the reflection undergoes a $180^{\circ}$ phase change. Using the alternating high and low index layers and quarter-wavelength thicknesses, the reflections from each layer become in phase and interfere constructively. This results in a high reflectivity at the design wavelength.

\subsection{Polarization and Ellipsometry}

Propagation of light can be described using Maxwell's equations, which describe the properties of electric and magnetic fields. These equations are:

$$
\begin{aligned}
& \nabla \cdot D=\rho \\
& \nabla \cdot B=0 \\
& \nabla \times E=-\frac{\partial B}{\partial t}
\end{aligned}
$$




$$
\nabla \times H=J+\frac{\partial D}{\partial t}
$$

where $\mathbf{E}$ and $\mathbf{B}$ the electric and magnetic fields, $\mathbf{D}$ is the electric flux density, and $\mathbf{H}$ is the magnetic field strength [12]. For an absence of free charges and assuming that the polarization vector $\mathbf{E}_{\mathbf{0}}$ lies perpendicular to the direction of propagation, equation 1.5 can reduce to:

$$
\nabla \cdot E=0
$$

Given equation 1.9, the polarization state of a wave can then be described by its components in any two orthogonal axes. These are the s-component (oscillating perpendicular to the plane of incidence) and $p$-component (oscillating parallel to the plane of incidence). The components of light reflected by an optical system are shown in figure 1.4.1 [13].

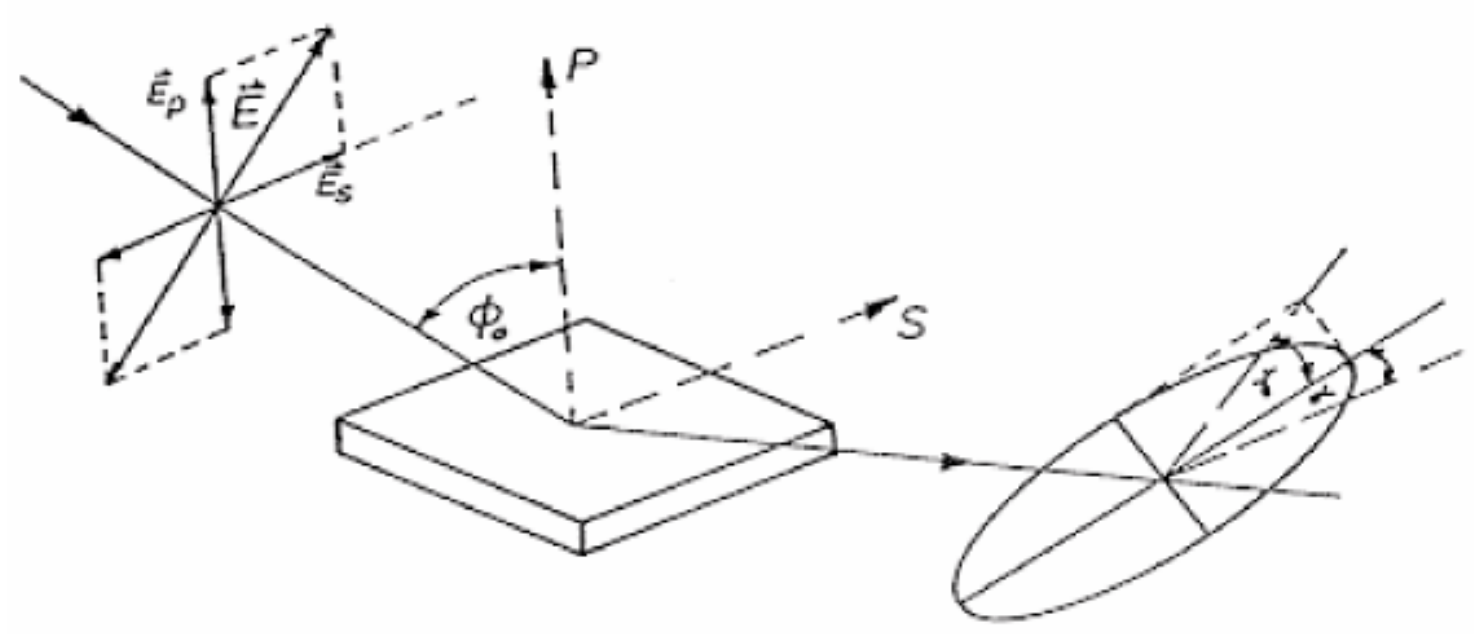

Figure 1.4.1 Polarization States Utilized by Ellipsometry [13] 
A method that utilizes these polarization states is Ellipsometry. Ellipsometry is a technique that uses polarized light to characterize optical systems. Ellipsometry measures the polarization state of light reflected or transmitted by an optical system and relates them to the fundamental equation of ellipsometry $[13,15]$ :

$$
\rho=\frac{R_{p}}{R_{s}}=\tan (\psi) e^{i \Delta}
$$

This equation measures the ratio of reflections in the $p$ and $s$ directions to determine the amplitude of reflected wave, $\tan (\Psi)$, and the phase of the reflected wave, $\Delta$. Ellipsometry is advantageous in that it is able to acquire these two terms of information, compared to traditional reflection measurements which can only give the amplitude of reflection at normal incidence [12]. Ellipsometry also does not require the use of mirrors or reference samples for background correction. This eliminates the error and guesswork associated with normalizing reflection data.

Variable Angle Spectroscopic Ellipsometry (VASE) will be used extensively in this work. Variable Angle Spectroscopic Ellipsometry uses ellipsometry data at multiple angles and wavelengths to better characterize optical systems. Variable Angle Spectroscopic Ellipsometry also allows for modeling of complex optical systems with precision accuracy. This is critical in the characterization of more complex structures like DBRs and other multi-film optical systems. A flow chart of an ellipsometry process is shown in figure 1.4.2 [14]. The optical system is first measured and then fit to an optical model. This is repeated until a good fit with a low mean squared error is obtained. Once a reasonable fit is obtained, parameters such as refractive index and thickness for materials can be determined. The thin film optical models used in this work typically employ the Cauchy dispersion formula, while bulk substrates like sapphire are often calculated using lookup tables. 


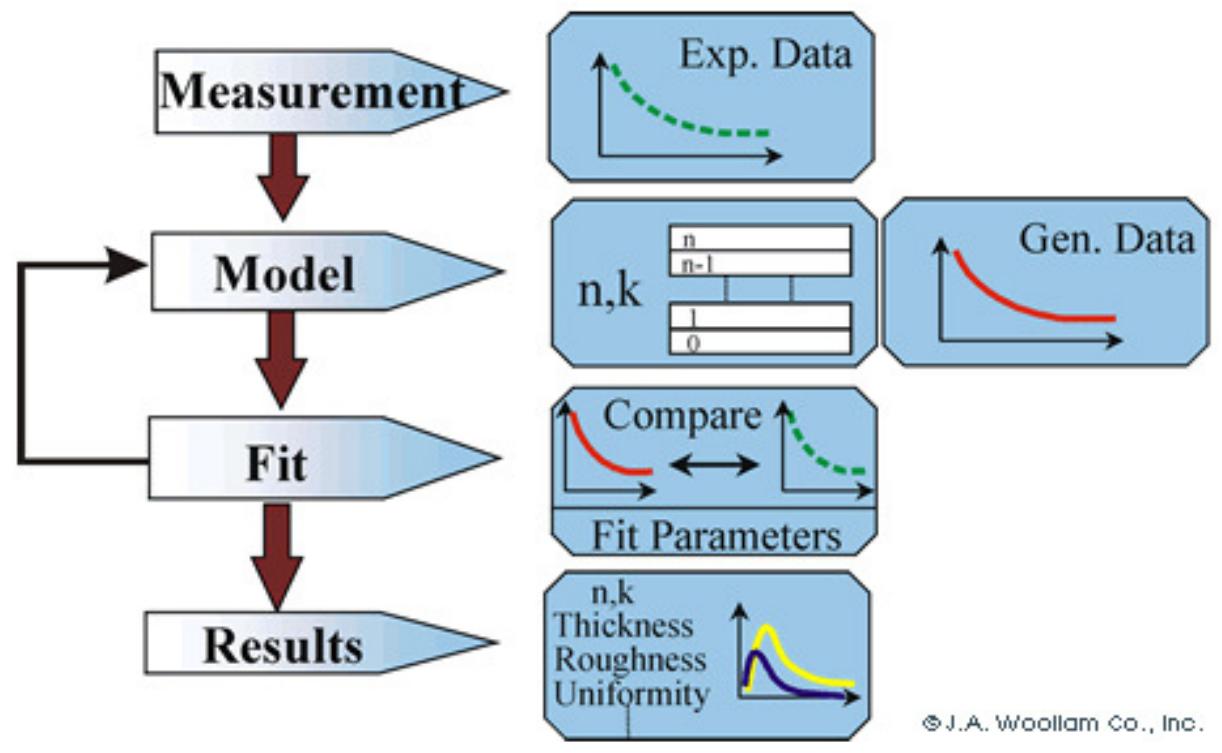

Figure 1.4.2 Flow Chart of Ellipsometry [14]

All ellipsometry data in this work was acquired using an M-2000U Variable Angle Spectroscopic Ellipsometer and modeled using WVASE32 software, both from J.A. Woollam Co. Inc.

\subsection{Motivation}

The motivation of this work is to grow, characterize, and simulate Distributed Bragg Reflectors made from III - N materials. Through characterization and simulation, future growths can be formulated to improve on the characteristics of previous growths. Problems such as strain and cracking can alter the refractive index of thin films and alter the overall efficiency of DBRs. Also, imprecise layer thicknesses can greatly alter the target wavelength and overall reflectivity of DBR structures. By studying previously grown structures, growth parameters and times can be altered to achieve a desired DBR structure.

High quality DBRs are needed for applications such as vertical-cavity surfaceemitting lasers (VCSELs), laser diodes, and light emitting diodes (LEDs). A schematic of a typical LED employing a DBR structure can be seen in figure 1.5.1 [16]. All these 
technologies require high quality mirrors to increase their efficiency and limit losses from light propagating in undesired directions. In addition, VCSELs often use DBRs on both sides of the active layer to compensate for the thin active layer. VCSELs are important optical sources used in applications like fiber-optic communication and consumer electronics [17]. VCSELs have the advantage over edge-emitting lasers in that they can have shorter cavity lengths on the order of the target wavelength. This allows VCSELs to have single-mode operation and be driven with much lower current than traditional edgeemitting lasers leading to less power consumption [3]. Since VCSEL DBRs must be able to be grown on the same crystal lattice as the active cavity, III - N DBRs are the obvious choice for any VCSEL with a III - N active layer.

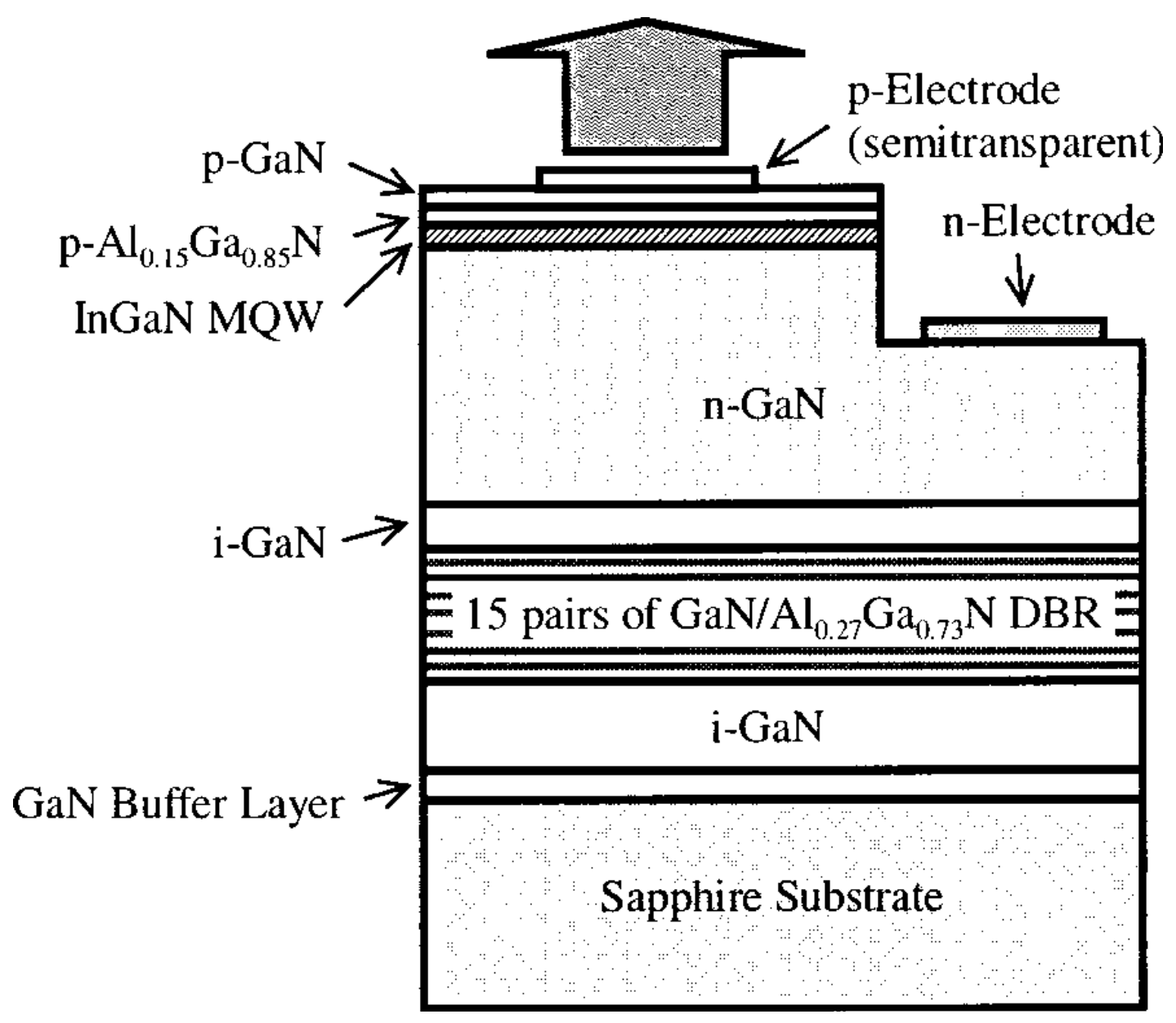

Figure 1.5.1 III - N LED Structure [16] 
One of the limiting factors in III - N VCSEL performance is the lack of high quality, crack free DBR structures to direct light into the active quantum well region. Extensive cracking and strain can result from the lattice mismatch between different III - N material layers and is increased due to the large number of layers required to create highly reflective DBRs. It has been reported that increases in strain and cracking can lead to an overall decrease in reflectivity [18]. Many different methods have been used to reduce cracking, including using ternary and quaternary alloys. While lattice mismatch can be decreased by using ternary and quaternary alloying, this results in a lower index contrast between the layers and decreases the overall reflectivity of the DBRs. The overall reflectivity can be increased by adding more bilayers; however, this increases production costs and the overall size of devices. This creates a need to improve film qualities without compromising the index contrast in order to achieve the best possible efficiency. 


\section{Chapter 2 Thin Film Characterization}

\subsection{Thin Films}

Thin films are layers of materials with thicknesses usually ranging from nanometers to several micrometers in thickness. Thin Films are often used for semiconductor and optical devices ranging from transistors and photovoltaic cells to simple optical coatings. Thin films can be produced using a variety of deposition methods, including those methods mentioned in section 1.2. Thin films are often advantageous over bulk devices in that they use less material and energy to produce, reducing their cost. In the case of photovoltaic cells, using less energy for production significantly decreases the energy payback time and, thus, increases their benefit to the environment [19]. Knowing the properties of a thin film is critical to understanding its applications and functionality. For thin films to be used in optical applications like those discussed here; thickness, refractive index, and bandgap are critical parameters that need to be determined to make efficient and reliable devices.

\subsection{Thickness}

Film thickness is a critical parameter in device design, especially when designing optical systems like DBRs and waveguides that require precision accuracy. Determining film thicknesses is critical in understanding device performance and establishing growth rates for future growths. Determining thickness uniformity over an entire wafer is important when fabricating multiple devices on a single wafer. There are many methods for determining film thickness, but from an optical perspective spectroscopy and ellipsometry are two important and widely employed methods [10, 16, 18, 29, 33]. 


\subsubsection{Spectroscopy}

Spectroscopy uses a range of wavelengths of light and their interaction with materials for characterization of materials. Using one or multiple spectrometers equipped with diffraction gratings or prisms, light can be broken down by wavelength and then used to characterize materials by measuring the interaction of the light with the material. Spectroscopy can be used to determine a thin film thickness by observing constructive and destructive interference due to light reflecting off the top and bottom of the film incidence as shown in figure 2.2.1:

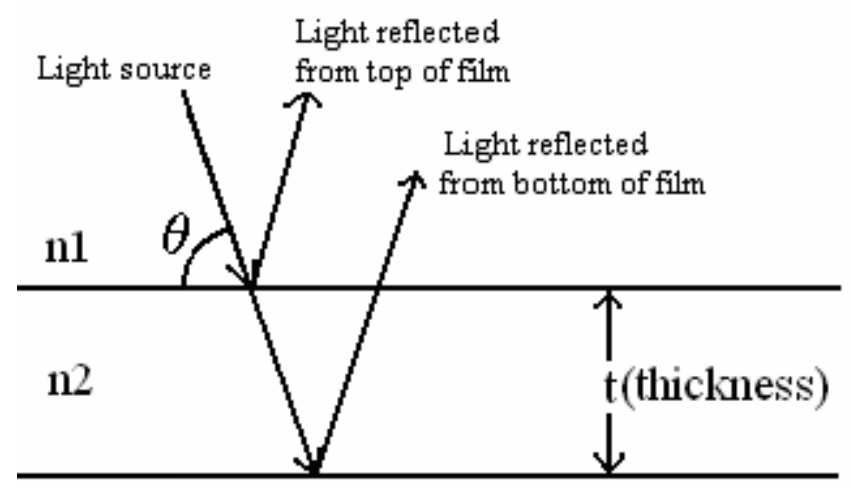

Figure 2.2.1 Thin Film Interference

This is typically performed at normal incidence $\left(\theta=90^{\circ}\right)$ for simplicity of setup and analysis. By monitoring the phase change between these two reflections and knowing the refractive index, the thickness can be extracted. An example of the reflection spectrum for a $1 \mu \mathrm{m} \mathrm{GaN} \mathrm{film} \mathrm{is} \mathrm{shown} \mathrm{in} \mathrm{figure} \mathrm{2.2.2.} \mathrm{When} \mathrm{the} \mathrm{two} \mathrm{reflections} \mathrm{are} \mathrm{in} \mathrm{phase,} \mathrm{they} \mathrm{have} \mathrm{a}$ higher reflectivity of around $30 \%$, while this drops to near $8 \%$ when the reflections are $180^{\circ}$ out of phase. At normal incidence, these peaks are predicted by the equation [20]:

$$
m \lambda_{0}=2 n t
$$


where $m$ are consecutive integer values. By plotting these integer values versus $2 n / \lambda$, the thickness can be extracted by finding the slope of the resulting plot as seen in figure 2.2.3.

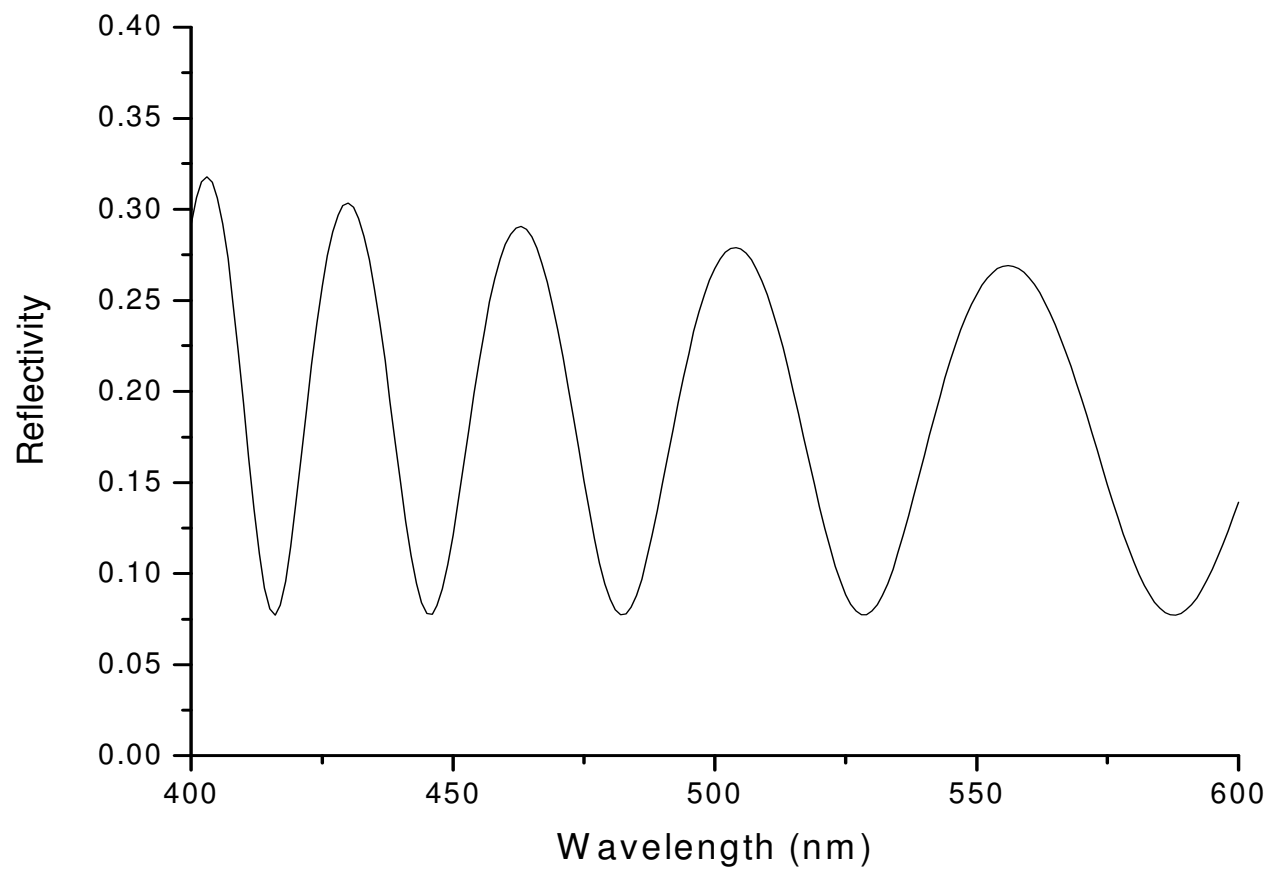

Figure 2.2.2 Reflectivity of a GaN Thin Film 


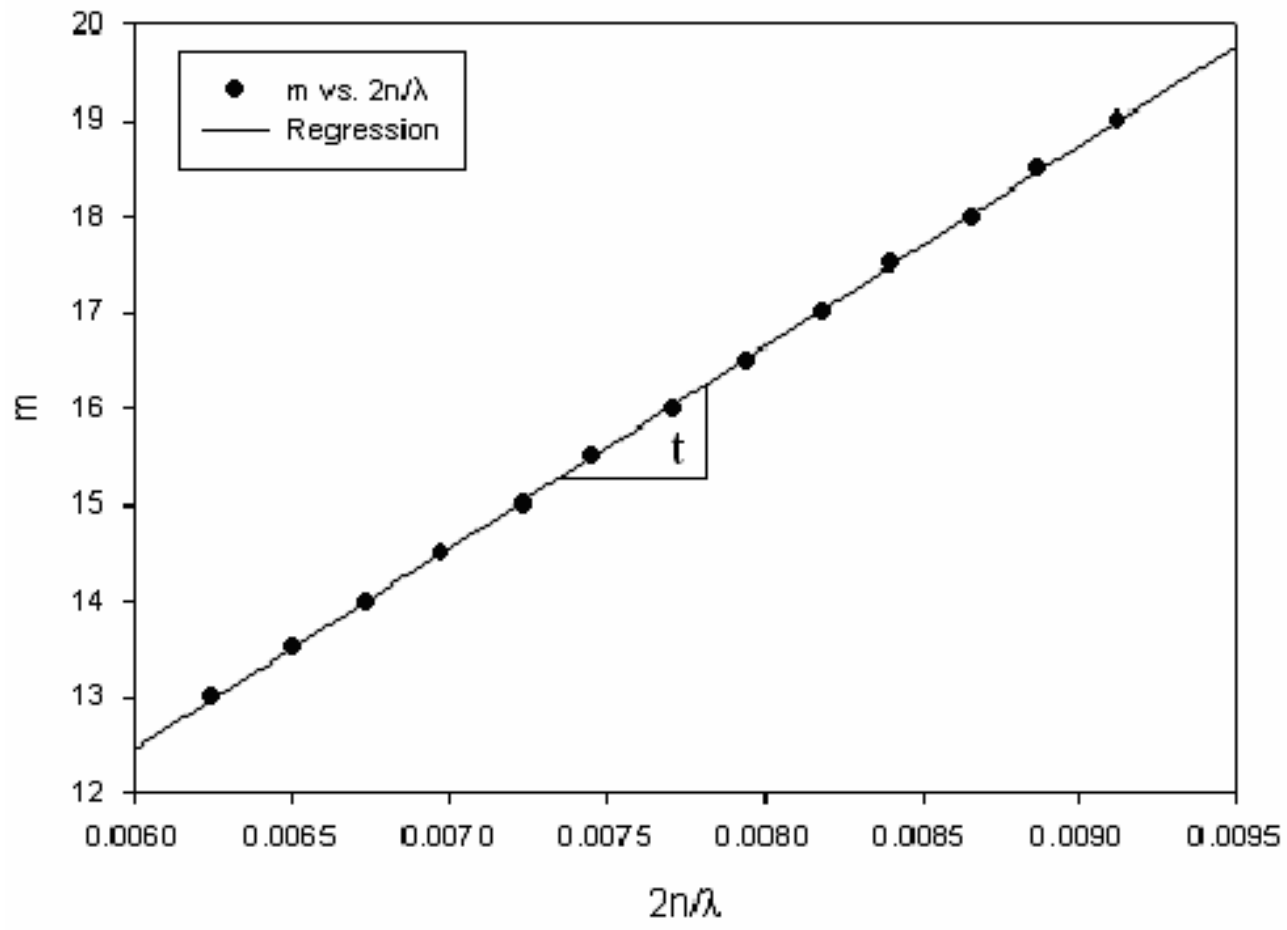

Figure 2.2.3 Linear Regression of Interference Fringes

This method works well for many thin films but has some limitations. First, films must be sufficiently thick in order to produce a number of observable interference peaks over the scan range for a good regression to be performed. For very thin films (those less than a few hundred nanometers), this technique is very ineffective. Second, the interference peaks are actually based on the product of the refractive index and thickness, so the refractive must be assumed based on literature or previous experiments. For thinner films or those with unknown refractive index, other methods like ellipsometry are required.

\subsubsection{Ellipsometry}

As discussed in chapter 1, ellipsometry is a method that uses the polarization state of light reflected or transmitted by an optical system in order to gain information about that system. Variable Angle Spectroscopic Ellipsometry (VASE) scans this information for multiple wavelengths and angles and returns raw data in terms of $\tan (\Psi)$ and $\cos (\Delta)$. From this raw data, a model like that shown in figure 2.2.4 is used and fit to the raw data. 


\begin{tabular}{|llr|}
\hline 2 & srough & $1.573 \mathrm{~nm}$ \\
\hline 1 & cauchy gan & $1612.669 \mathrm{~nm}$ \\
\hline 0 & al203 & $1 \mathrm{~mm}$
\end{tabular}

Figure 2.2.4 Sample Ellipsometry Model

Using the chosen model, the WVASE32 software fits the theoretical data to the raw data acquired in the scan and uses a mean square error (MSE) calculation to minimize the error to best fit the raw data. Equation 2.2 shows this MSE equation [21]:

$$
M S E=\frac{1}{2 N-M} \sum_{i=1}^{N}\left[\left(\frac{\psi_{i}^{\bmod }-\psi_{i}^{\exp }}{\sigma_{\psi}^{\exp }}\right)^{2}+\left(\frac{\Delta_{i}^{\bmod }-\Delta_{i}^{\exp }}{\sigma_{\Delta}^{\exp }}\right)^{2}\right]
$$

where $\mathrm{M}$ is the number of unknowns and $\mathrm{N}$ is the number of wavelengths scanned. In addition to the MSE and optimized model parameters, the software also plots the model data for comparison to the raw data as shown in figure 2.2.5. 


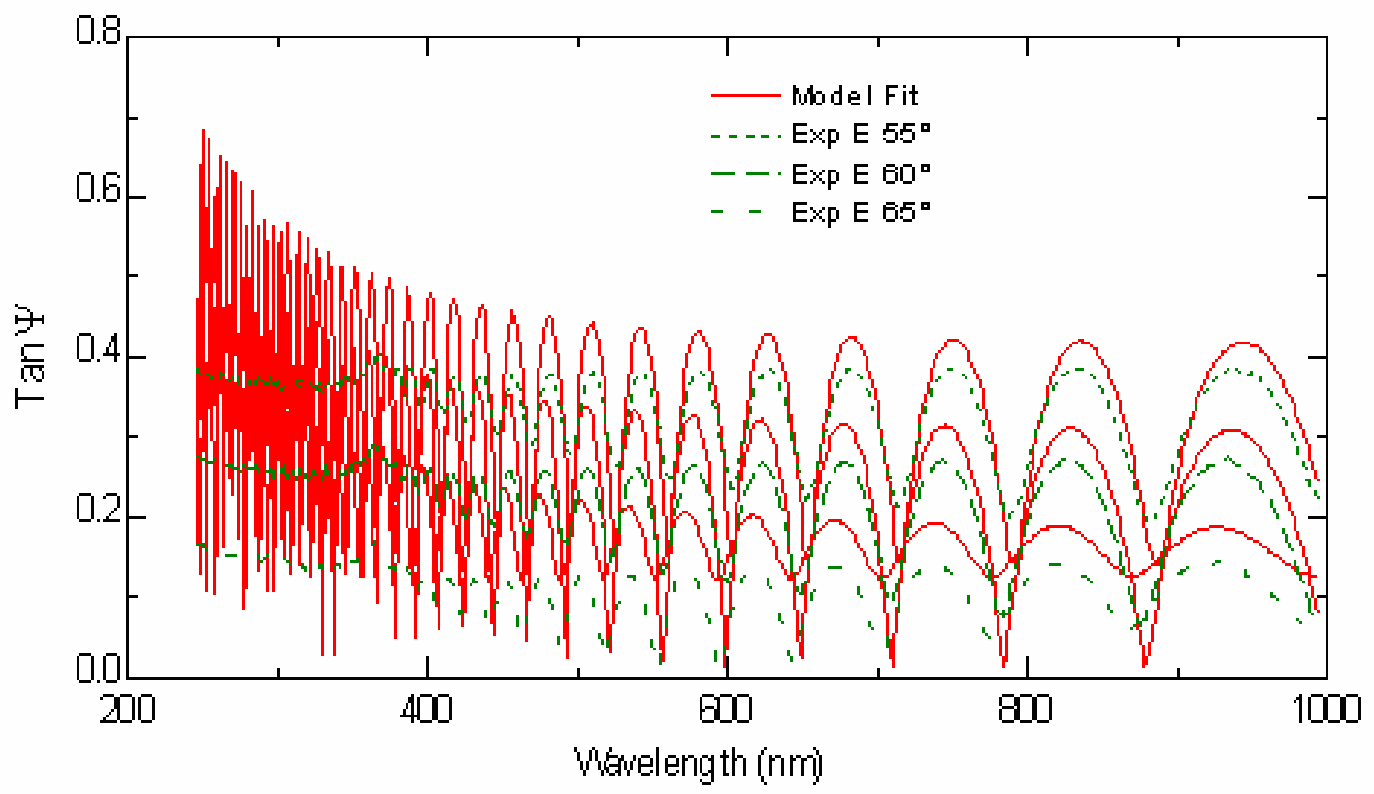

Figure 2.2.5 VASE Raw Data and Model Fit

This method is much more effective than conventional spectroscopy for many reasons. For one, films as thin as a few nanometers can be fit. This would be nearly impossible to do with any degree of accuracy with traditional spectroscopy. Also, there is flexibility to fix the refractive index to a given value or have it fit along with the thickness, a method that will be discussed later. This method is crucial when a films refractive index may not be known to a degree of certainty. Also, additional parameters like surface roughness can be added to get a more complete view of the optical system. Finally, multiple film systems can be fit much more accurately, which will become crucial when doing more complex optical systems like DBRs.

\subsubsection{Thickness Uniformity}

Another advantage of Variable Angle Spectroscopic Ellipsometry and the WVASE32 software is that it allows you to scan over the surface of a wafer to determine the thickness uniformity. This feature allows the creation of 2-dimensional and 3dimensional surface maps of an entire wafer. Examples of these are shown in figure 2.2.6 
(2-dimensional) and figure 2.2.7 (3-dimensional). As can be seen, the thickness of a film can vary greatly over a wafer and does not always deviate uniformly. These maps contain much more information about the film and better characterize the quality of growth or deposition over an entire wafer. They can also better predict and explain how devices might perform due to their positioning on the chip.

Thick.1
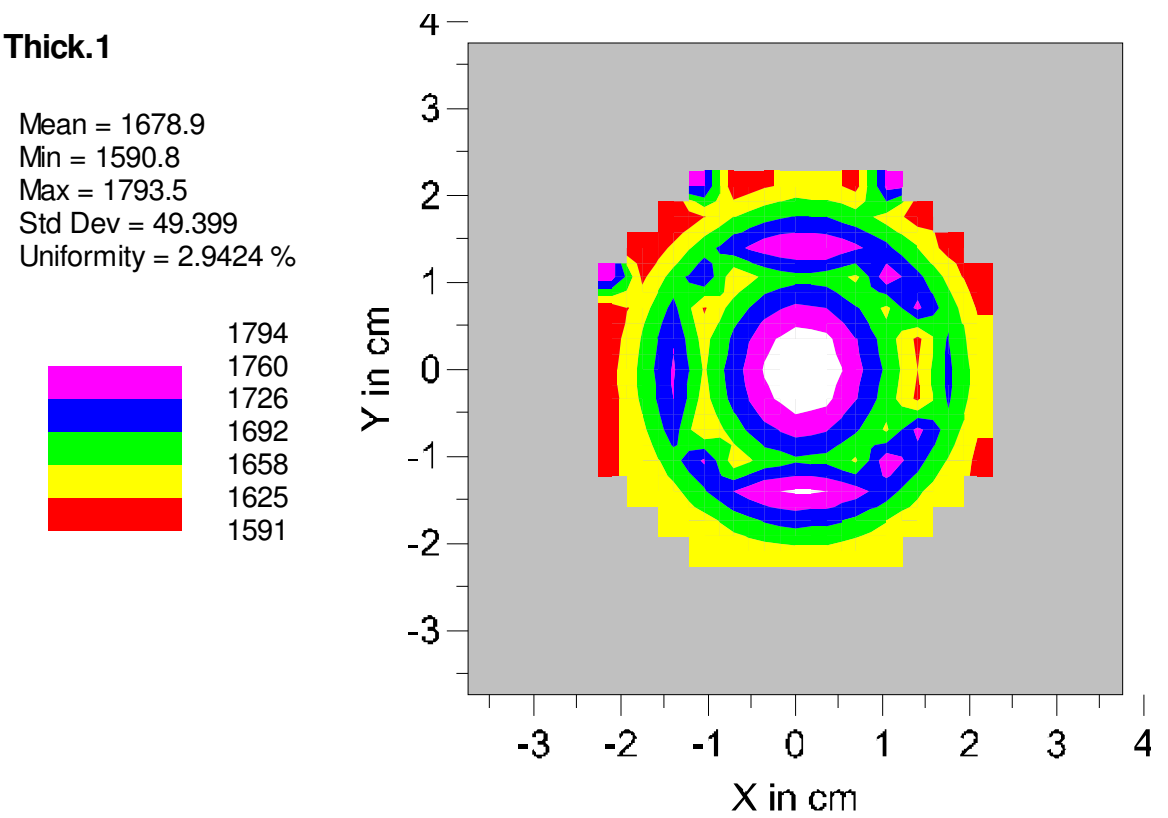

Figure 2.2.6 2-Dimensional Surface Map 


\section{Thick.1}
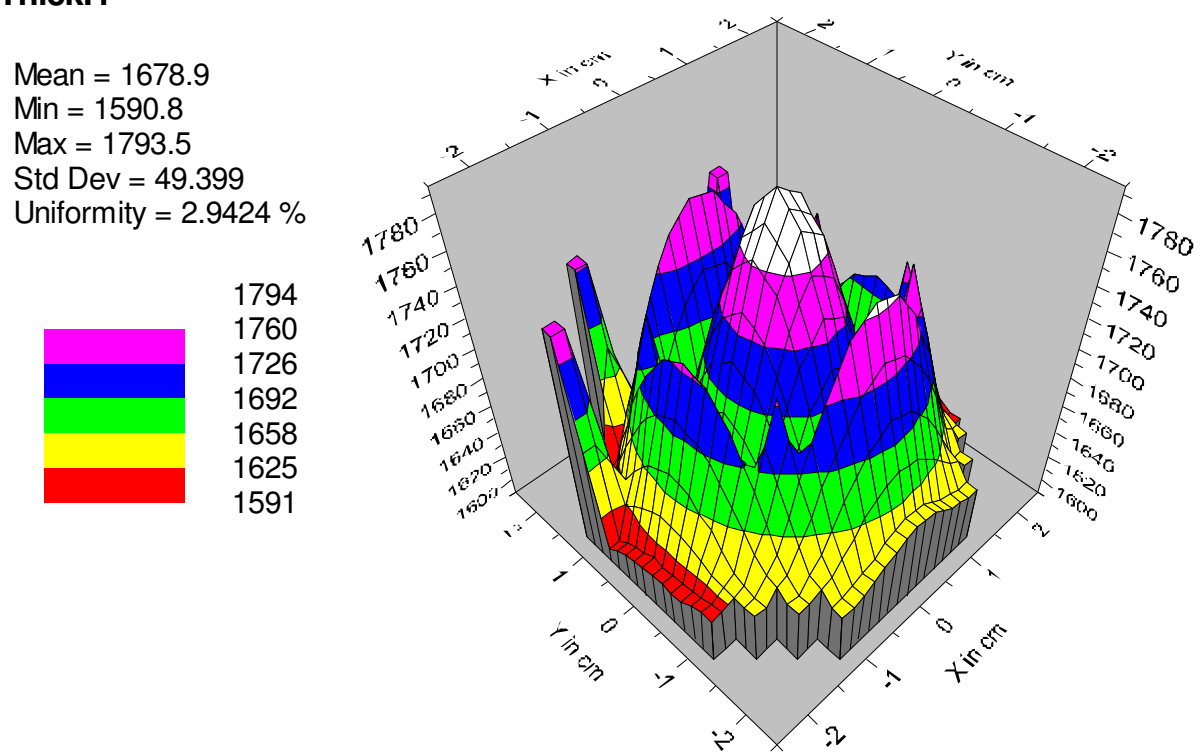

Figure 2.2.7 3-Dimensional Surface Map

\subsection{Refractive Index}

\subsubsection{Light in a Medium}

Refractive index is the measure of how an electromagnetic wave, light for example, travels in a medium. Refractive index is the related to the permittivity and permeability of a material by the equation:

$$
n=\sqrt{\frac{\varepsilon \mu}{\varepsilon_{0} \mu_{0}}}
$$

When entering a medium other than vacuum, a light wave will see a decrease in its velocity, dependent on the refractive index of that medium. This refractive index (n) is given as the ratio of this velocity (v) to the speed of light in vacuum (c) by: 


$$
n=\frac{c}{v_{n}}
$$

While the velocity of the wave changes, the frequency remains the same for obvious reasons. This leads to the fact that the wavelength of the wave also changes as a result of the change in velocity following the equation:

$$
\lambda_{n}=\frac{v_{n}}{f}
$$

Substituting equation 2.2 into 2.1, the wavelength of light in a medium can be found in relation to that medium's refractive index and the wavelength of the light in vacuum $\left(\lambda_{0}\right)$ :

$$
\lambda_{n}=\frac{\lambda_{0}}{n}
$$

Knowing the wavelength of light in a medium will become important when designing optical systems like determining the quarter wavelength thick layers used in Bragg Reflectors.

When a light wave enters one medium from another at an angle other than normal incidence, it is refracted at a new angle as shown in figure 2.3.1 [22]. This is due to the change in speed of the wave as it enters the new medium. This angle of refraction is dependent on the refractive index of the two mediums. Snell's law (equation 2.5) is the formula for determining this new angle of refraction

$$
n_{1} \sin \theta_{1}=n_{2} \sin \theta_{2}
$$

Knowing the refractive index of the two mediums and the angle of the incident wave, the angle of the refracted wave can be determined. 


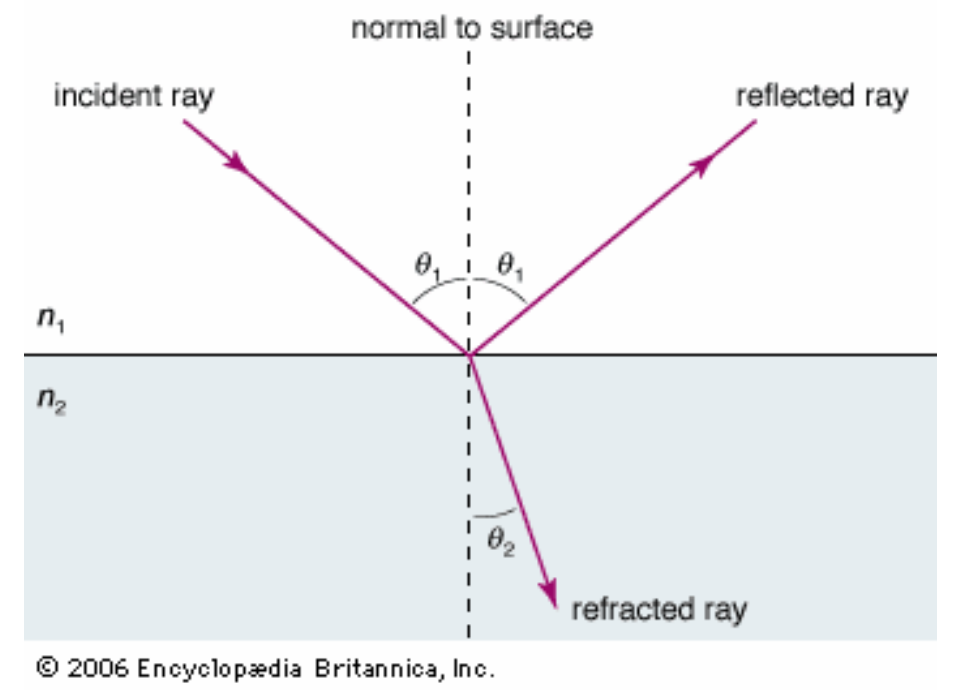

Figure 2.3.1 Snell's Law [22]

\subsubsection{Refractive Index Modeling}

Refractive index varies depending on the wavelength or energy of light. The wavelength of light is related to its energy by the equation:

$$
E_{\lambda}(e V)=h v \cong \frac{1240}{\lambda_{n m}}
$$

This refractive index variation becomes more prominent for shorter wavelengths of light, especially where the light's energy approaches that of the bandgap of the material [23]. While this effect varies for each material, it can be approximated using the Cauchy dispersion equation:

$$
n=A+\frac{B}{\lambda^{2}}+\frac{C}{\lambda^{4}}
$$


where A, B, C are constants dependant on the material. This model is sufficient for modeling the refractive index over the ultraviolet, visible, and infrared spectrum for all light with energies below the bandgap of the material; however, it does not account for light with energies above the bandgap. This is due to the refractive index actually being complex.

\subsubsection{Complex Refractive Index}

While the refractive index, $\mathrm{n}$, describes the way light travels through and is refracted by a medium, it is only part of the story. The refractive index of a material is actually a complex quantity. At energies much less than the bandgap, only the real part of the refractive index is important; but at energies approaching and above the bandgap, the imaginary part of the refractive index comes into play. This imaginary part is the extinction coefficient, $\mathrm{k}$, and causes the complex equation for refractive index to become:

$$
\tilde{n}=n+i k
$$

The extinction coefficient results from light having sufficient energy to create photon-induced electronic transitions [24]. Light is then able to be absorbed by the film and not just reflected or transmitted. This will be discussed in section 2.4.

\subsubsection{Refractive index in III - N films}

The refractive indices of III $-\mathrm{N}$ materials have been widely reported [25]. While these values provide a good baseline for refractive index, variations may exist due to reasons such as growth parameters, crystal quality, and doping. There can also be variations in refractive index due to stress and strain, which will be discussed in later chapters. Tertiary and Quaternary alloys have less defined refractive indices because they have been studied less and exact film composition is often not known. For these reasons and other, 
refractive indices often need to be determined. This can be done by again utilizing ellipsometry. In addition to fitting the thickness, the refractive index can be fit using a Cauchy dispersion formula as a model. This model is very effective for refractive index at energies below the bandgap. For energies above the bandgap, the refractive index can be directly extracted from the raw data since there are no secondary reflections as the film absorbs light entering it.

Figure 2.3.2 shows the real part of the refractive index of a GaN thin film found using ellipsometry as compared to literature. The refractive index of our MOVPE grown film is slightly higher than that predicted by literature; however, this seems to be consistent for most of our GaN films. It can also be seen that the refractive index peaks around the bandgap, a fact which will be later used in determining bandgap.

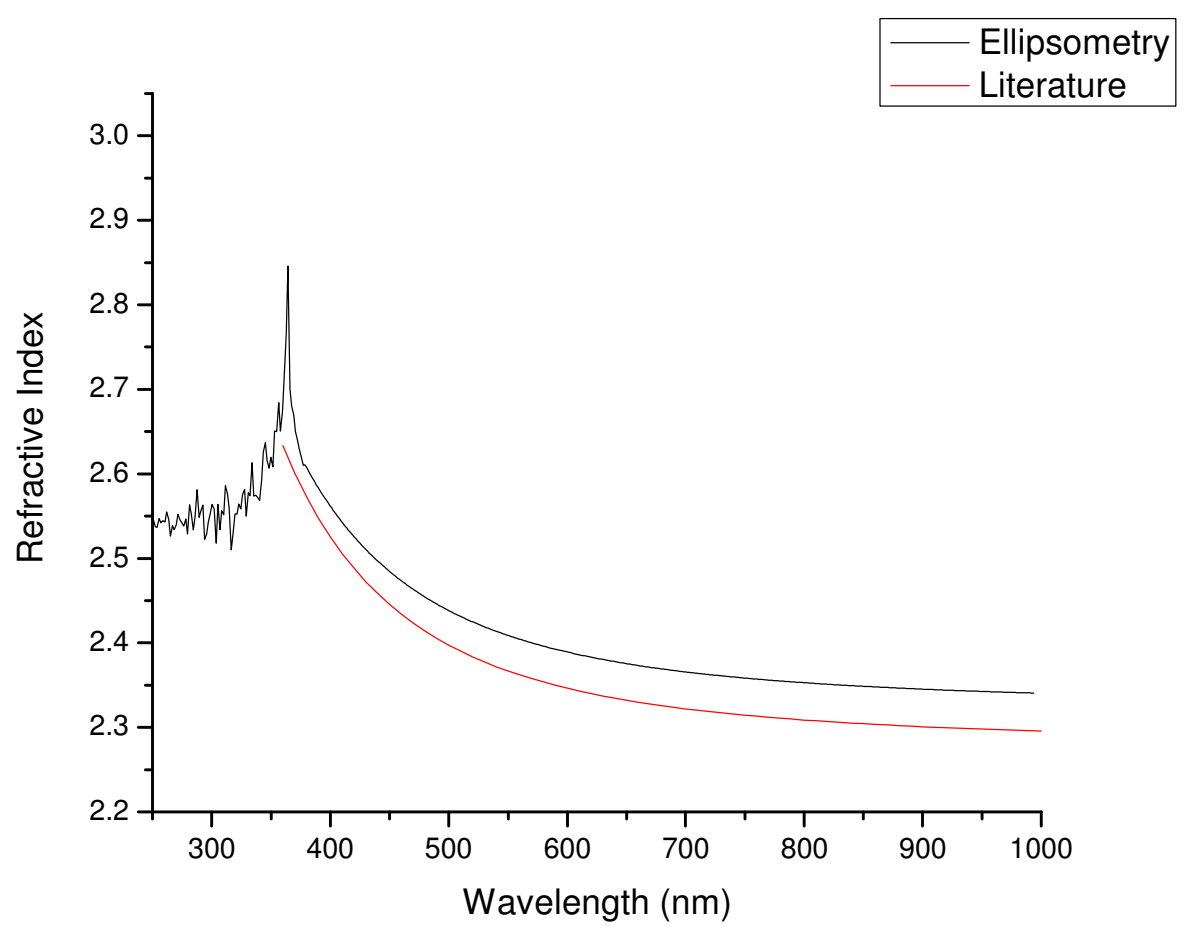

Figure 2.3.2 Refractive Index of a MOVPE Grown GaN Thin Film

The extinction coefficient, or imaginary part of the refractive index, can also be found using ellipsometry. As seen in figure 2.3.3, the extinction coefficient is fairly 
consistent with that reported in literature for energies higher than the bandgap [25]. For energies below the bandgap, the graphs do not agree; however, these transition values were not fully listed by literature and had to be interpolated. The values obtained in this work agree with data obtained using transmission spectroscopy and are also consistent with values found by others $[21,26]$. It can also be seen that there is an apparent difference in the extinction coefficient between the two films. The thicker film absorbs slightly more in the transition region as the light approaches the bandgap. This is due to the increased optical path length the light might travel and is not likely dependent on material properties.

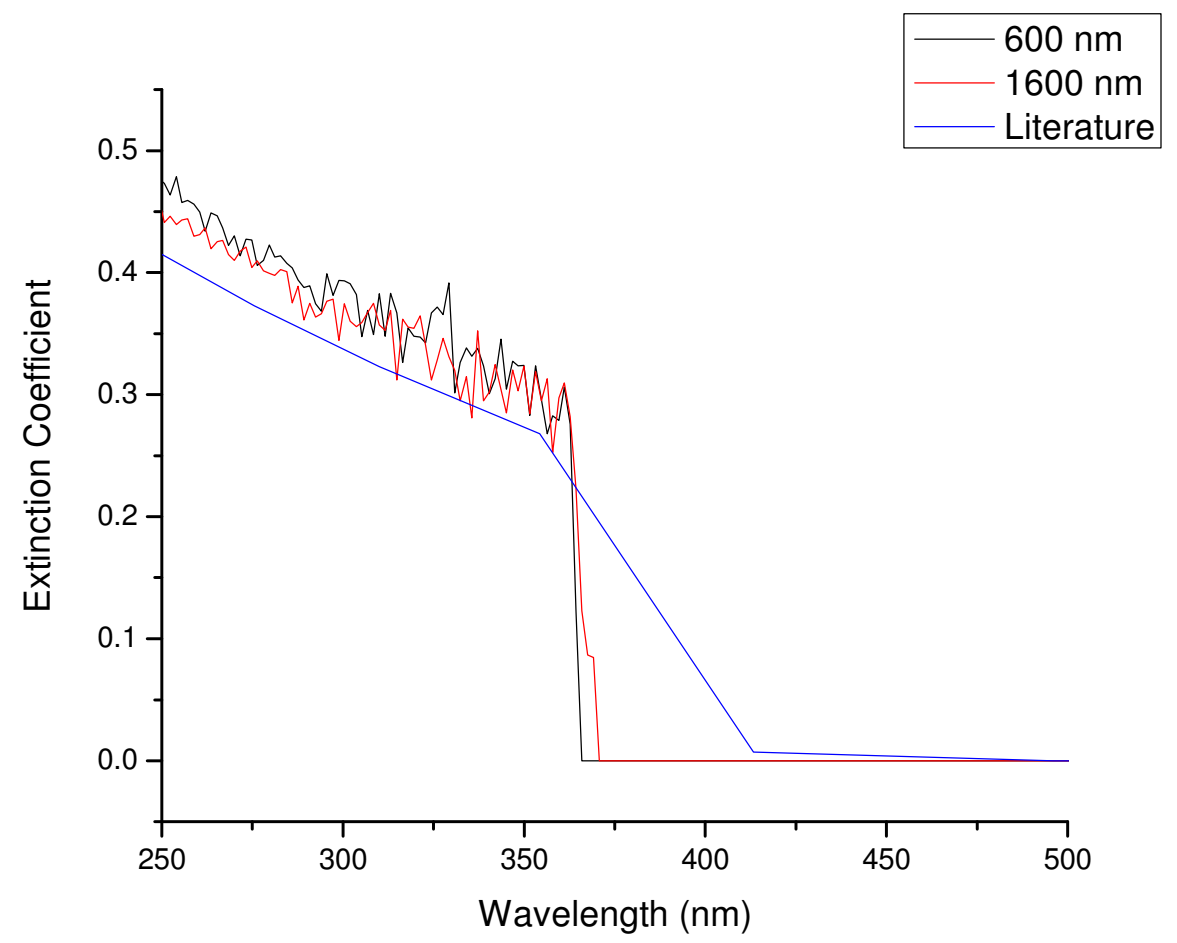

Figure 2.3.3 Extinction Coefficients of MOVPE Grown GaN Thin Films 


\subsection{Bandgap}

\subsubsection{Absorption}

Absorption results when light has sufficient energy to create photon-induced electronic transitions. This is described by the absorption coefficient and is related to the extinction coefficient by [23]:

$$
\alpha=\frac{4 \pi k}{\lambda}
$$

For direct bandgap materials like III - N semiconductors, these transitions mostly occur at bands of the same wave vector $(\mathrm{k}=0)$. Figure 2.4.1 shows these allowed transitions [23].

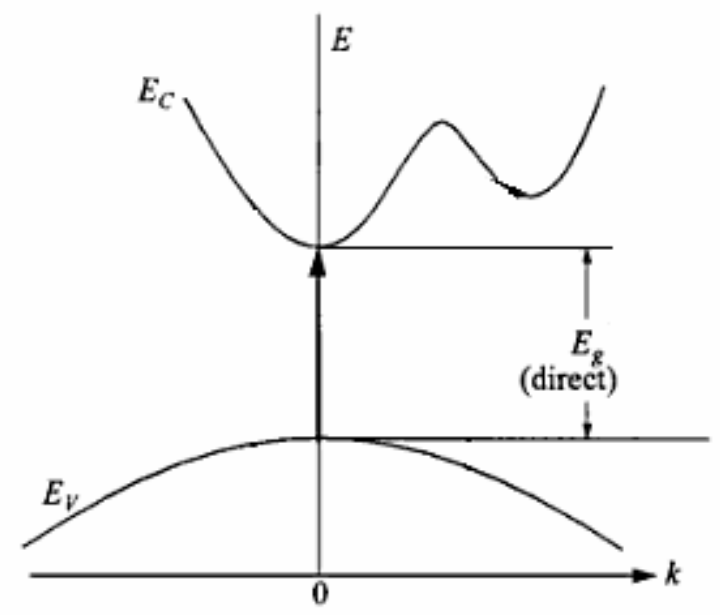

Figure 2.4.1 Allowed Direct Optical Transitions [23]

For direct bandgap semiconductors near the bandgap, the absorption coefficient can be approximated as: 


$$
\alpha \propto h v-E_{g}
$$

where $h v$ is the photon energy [23]. The absorption coefficient is effectively zero for energies far below the bandgap and then increases drastically at energies near the bandgap before beginning to level off above the bandgap. This transition region can be used to determine the bandgap of the thin film.

\subsubsection{Spectroscopy}

The absorption of a thin film can be related to the power of light going into and being transmitted through the film by the equation:

$$
P_{\text {oUT }}=P_{\text {IN }} e^{-\alpha x}
$$

By rearranging the equation, taking the natural log of each side, and squaring result; the square of this absorption coefficient can be related percentage of light transmitted through a film by:

$$
\alpha^{2} \propto \ln ^{2}\left(\frac{P_{O U T}}{P_{I N}}\right)
$$

By knowing the percentage of light transmitted through a sample, this relation can be utilized to determine the bandgap. Figure 2.4.2 shows the square of the absorption coefficient for a GaN film determined using transmission. By performing a linear regression of the transition region, the bandgap can be determined by finding the $\mathrm{x}$ intercept of the regression. The value for bandgap found here, $3.394 \mathrm{eV}$, is consistent with that found in literature.

This technique was also used on InGaN Multi Quantum Well (MQW) samples as shown in figure 2.4.3; however, it was found to be ineffective. The difference in apparent bandgaps for these samples was found to be largely dependent on the number of MQWs as 
opposed to Indium concentration. This was a result of the total InGaN thickness being different, which caused the penetration depth of photons to come into play. Also, as the bandgap of GaN was approached, the GaN film on which the MQWs were grown became the dominant mechanism for absorption, skewing the transition region and effective bandgap.

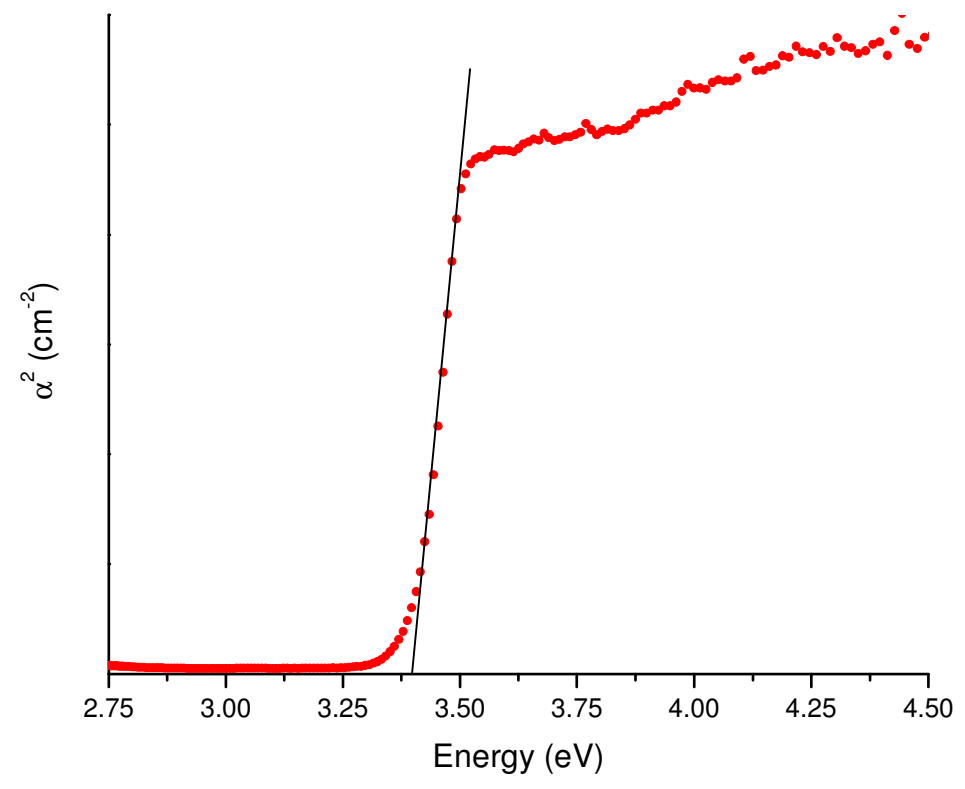

Figure 2.4.2 Square of Absorption Coefficient vs. Photon Energy for a GaN Film 


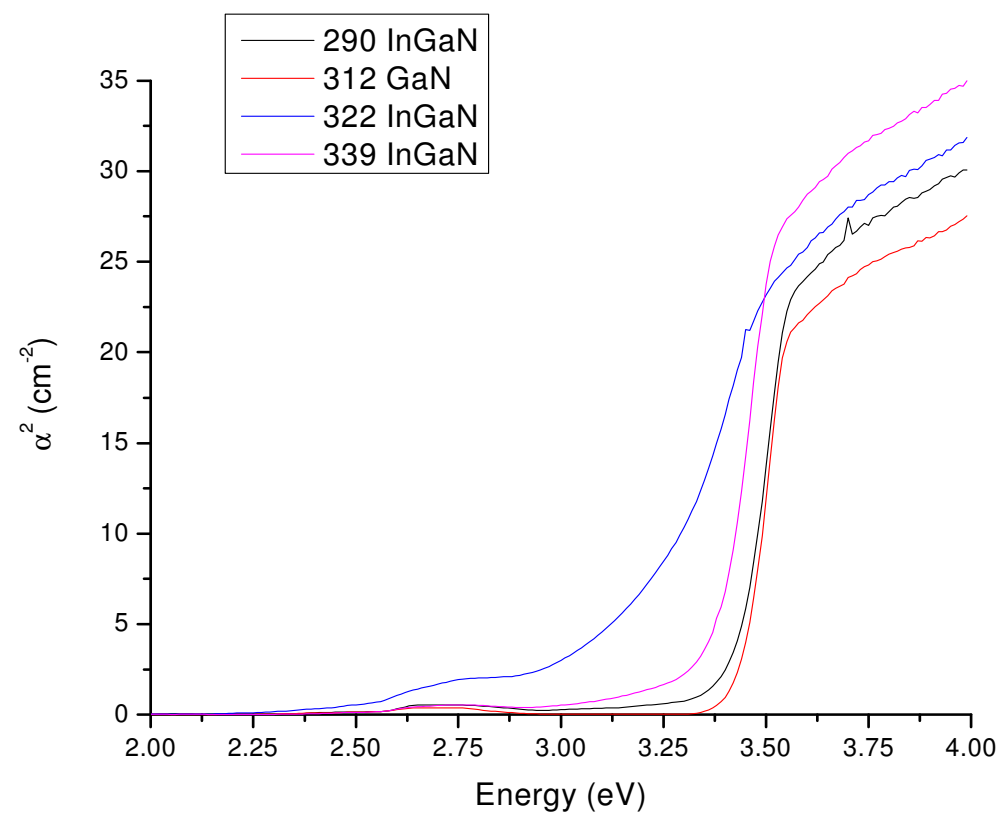

Figure 2.4.3 Square of Absorption Coefficient vs. Photon Energy for InGaN Films

The limitation of spectroscopy on multiple film optical systems again came into play when attempting transmission on $\mathrm{AlGaN}$ and AlN samples. Since these samples were grown on $\mathrm{GaN}$ substrates, the $\mathrm{GaN}$ absorbed all light at energies below where the $\mathrm{AlGaN}$ absorption came into play, resulting in the AlGaN layers having no effect on the absorption detected. Due to this problem, other techniques such as ellipsometry were used to determine the bandgap of AlGaN films.

\subsubsection{Ellipsometry}

Since traditional spectroscopy was ineffective in determining the bandgap of AlGaN films grown on GaN substrates, ellipsometry was again used. Two methods were utilized for doing this. First, the extinction coefficient was extracted using ellipsometry and then used to determine the absorption coefficient. The absorption coefficient was then used to determine the bandgap using the steps described in section 2.4.2. The other method was to directly extract if from the raw data for the refractive index. Figure 2.4.4 shows the 
refractive index raw data for an $\mathrm{Al}_{.7} \mathrm{Ga}_{.93} \mathrm{~N}$ film. By determining the peak in the refractive index, the bandgap can be determined. The values found here agreed with those found using the extinction coefficient from ellipsometry as well as those able to be determined using transmission.

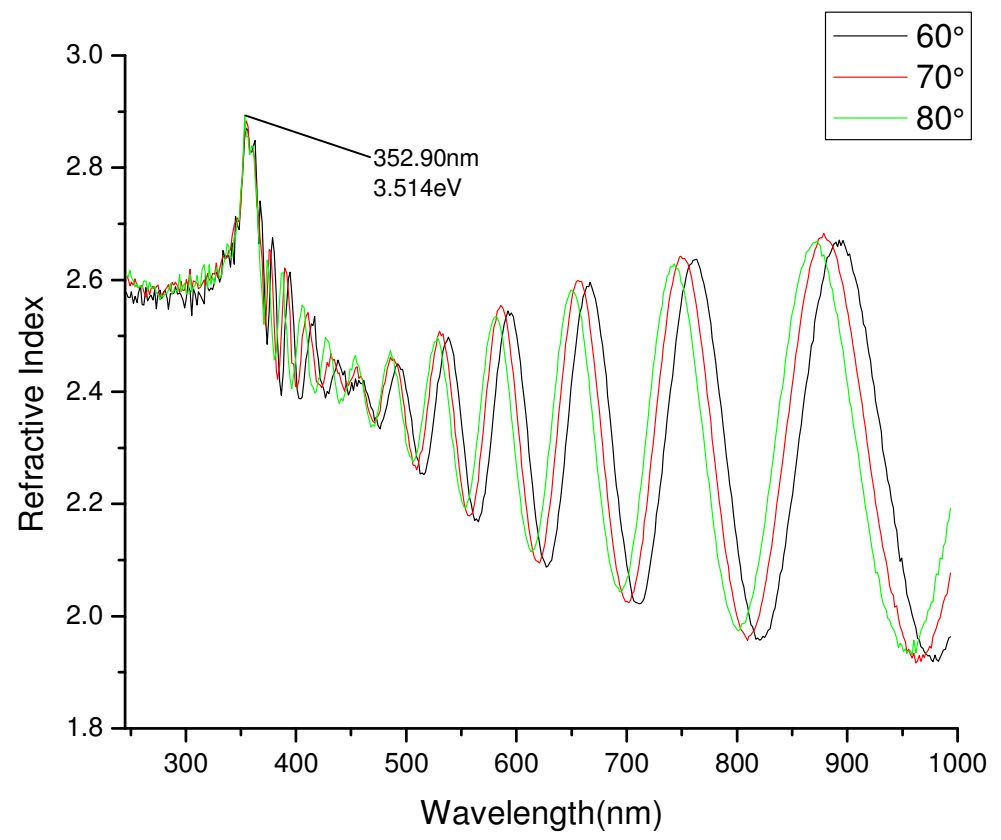

Figure 2.4.4 Refractive Index Raw Data for an AlGaN Film

This method was performed on $\mathrm{AlGaN}$ thin films with different Aluminum concentrations and plotted in Figure 2.4.5. The peak location appeared to follow a linear trend. This, however, is unlikely as extrapolating this data incorrectly predicts the bandgap for AlN. Also, literature suggests that some bowing occurs and the relation is indeed not linear [1]. Figure 2.4.6 compares the results found here with those in literature using techniques such as transmission, photoluminescence and photoreflectance [27, 28]. The bowing parameter range suggested by literature is also plotted [1]. The results found here agree well with those found by other groups. The values found suggest that the bandgap 
increases as the aluminum concentration in AlGaN increases. Values found here and in literature suggest that bowing does occur and the transition in bandgap from GaN to AlN is not linear.

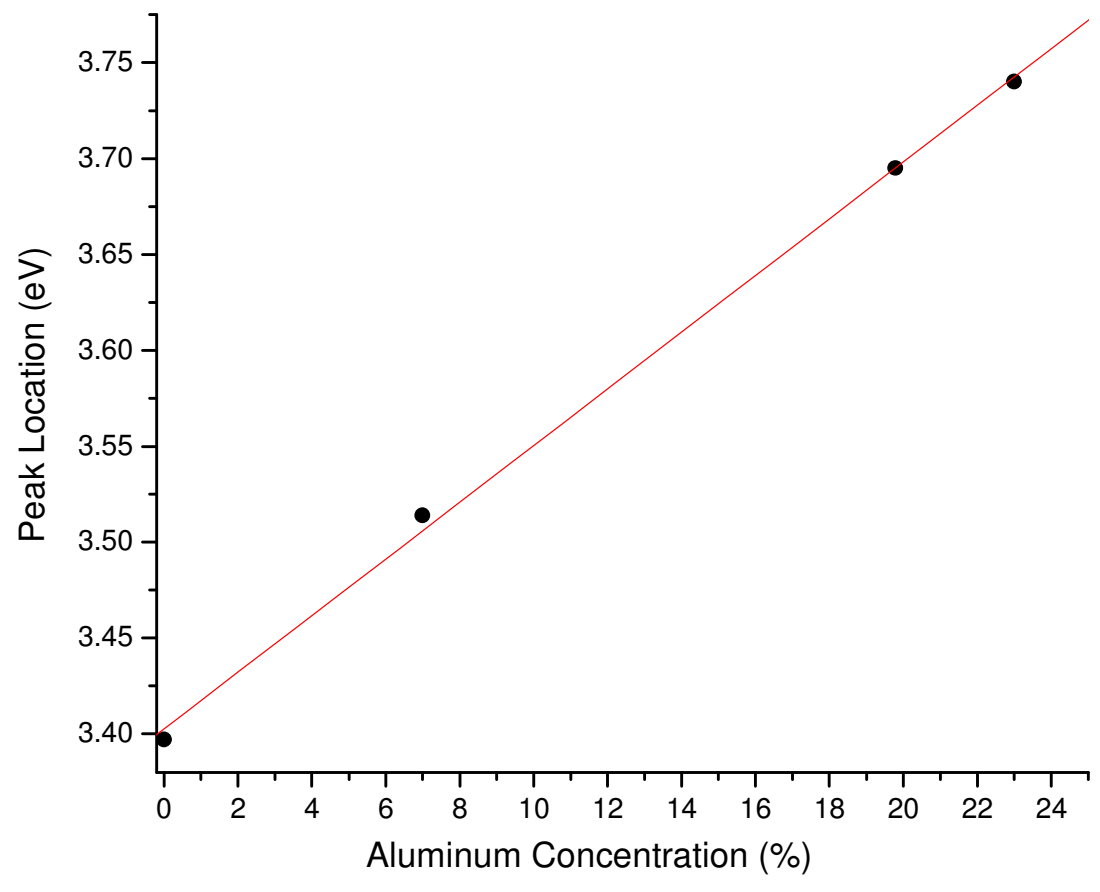

Figure 2.4.5 Peak Location vs. Aluminum Concentration 


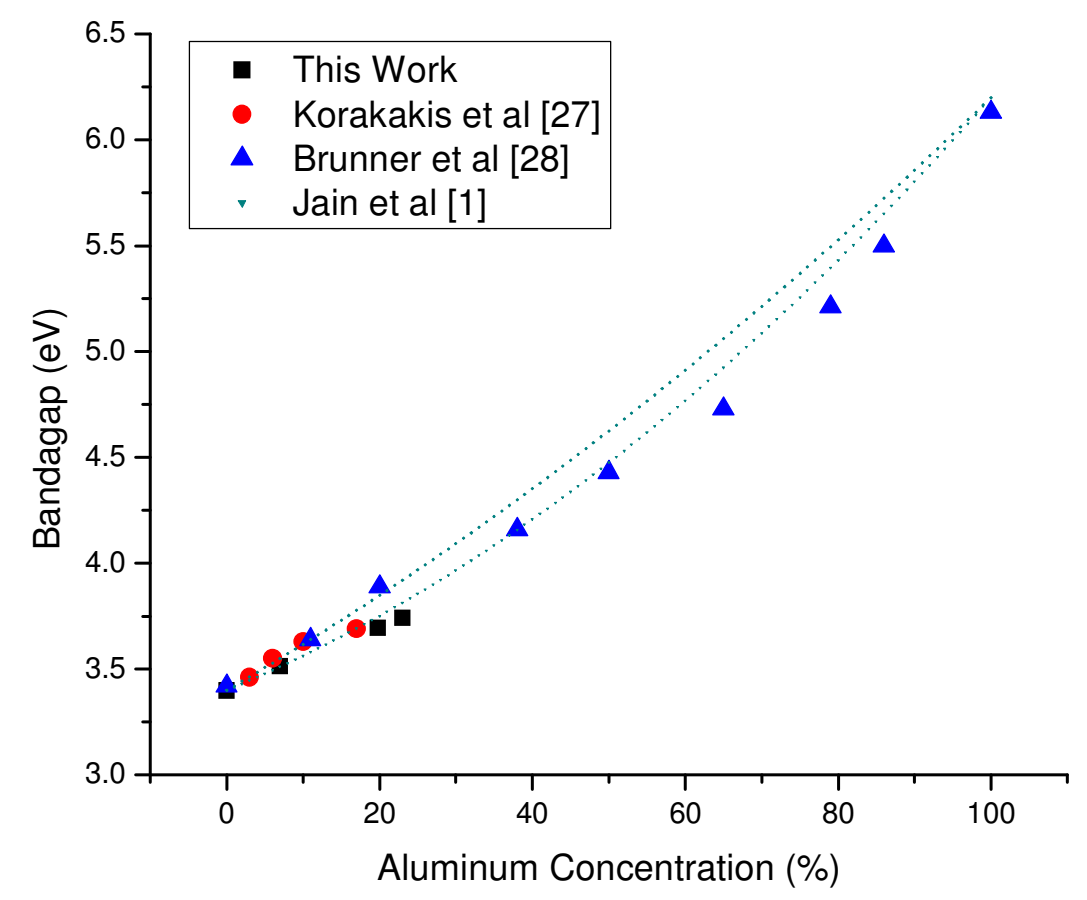

Figure 2.4.6 Comparison of Bandgaps vs. Aluminum Concentration in AlGaN films

\subsection{Summary}

III - $\mathrm{N}$ thin films were characterized to determine properties like film thickness, refractive index, and bandgap. Determining these properties is crucial in understanding and optimizing growth parameters. Characterization will be expanded from single film to multiple film optical systems in order to characterize, simulate, and develop Distributed Bragg Reflectors. 


\section{Chapter 3 Distributed Bragg Reflector Simulation Methods}

\subsection{Introduction}

Simulating Distributed Bragg Reflectors is important in predicting and performance of DBR growths as well as characterizing their actual performance. Simulations will be performed using the Transmission Matrix Method (TMM) calculated using MATLAB as well as MEEP, a simulation package developed at the Massachusetts Institute of Technology (MIT).

\subsection{Transmission Matrix Method in MATLAB}

\subsubsection{Transmission Matrix Method}

The Transmission Matrix Method has been shown to be effective in modeling DBR reflectivity [29]. Using this method, the total reflectance can be modeled as [30]:

$$
\begin{aligned}
& t=\frac{\lambda}{4 n} \\
& {\left[\begin{array}{l}
B \\
C
\end{array}\right]=\prod_{r=1}^{q}\left[\begin{array}{cc}
\cos (t) & \frac{i \sin (t)}{n} \\
i n \sin (t) & \cos (t)
\end{array}\right]\left[\begin{array}{c}
1 \\
n_{\text {subs }}
\end{array}\right]} \\
& Y=\frac{C}{B}
\end{aligned}
$$




$$
R=\left(\frac{1-Y}{1+Y}\right)\left(\overline{\frac{1-Y}{1+Y}}\right)
$$

where $t$ is the film thickness, $n$ is the film refractive index, and $R$ is the reflectivity. These calculations use film thicknesses and refractive indices to calculate the optical impedance and from this, the overall reflectivity of a DBR system. Using MATLAB, a loop can be created to perform this calculation for every layer in the DBR structure and find the total reflectivity of the system

\subsubsection{Refractive Indices}

MATLAB simulations were carried out for ideal quarter-wave thick AlN and GaN layers using refractive indices found in literature. The refractive Indices for AlN and GaN were first interpolated from literature and plotted as shown in figure 3.2.1 [25]. They were then fit with the Cauchy-dispersion model to obtain equations 3.5 and 3.6.

$$
\begin{aligned}
& n_{\text {GaN }}=2.2796+\frac{.01175}{\lambda^{2}}+\frac{.0044175}{\lambda^{4}} \\
& n_{\text {AlN }}=2.1071-\frac{.01881}{\lambda^{2}}+\frac{.001952}{\lambda^{4}}
\end{aligned}
$$




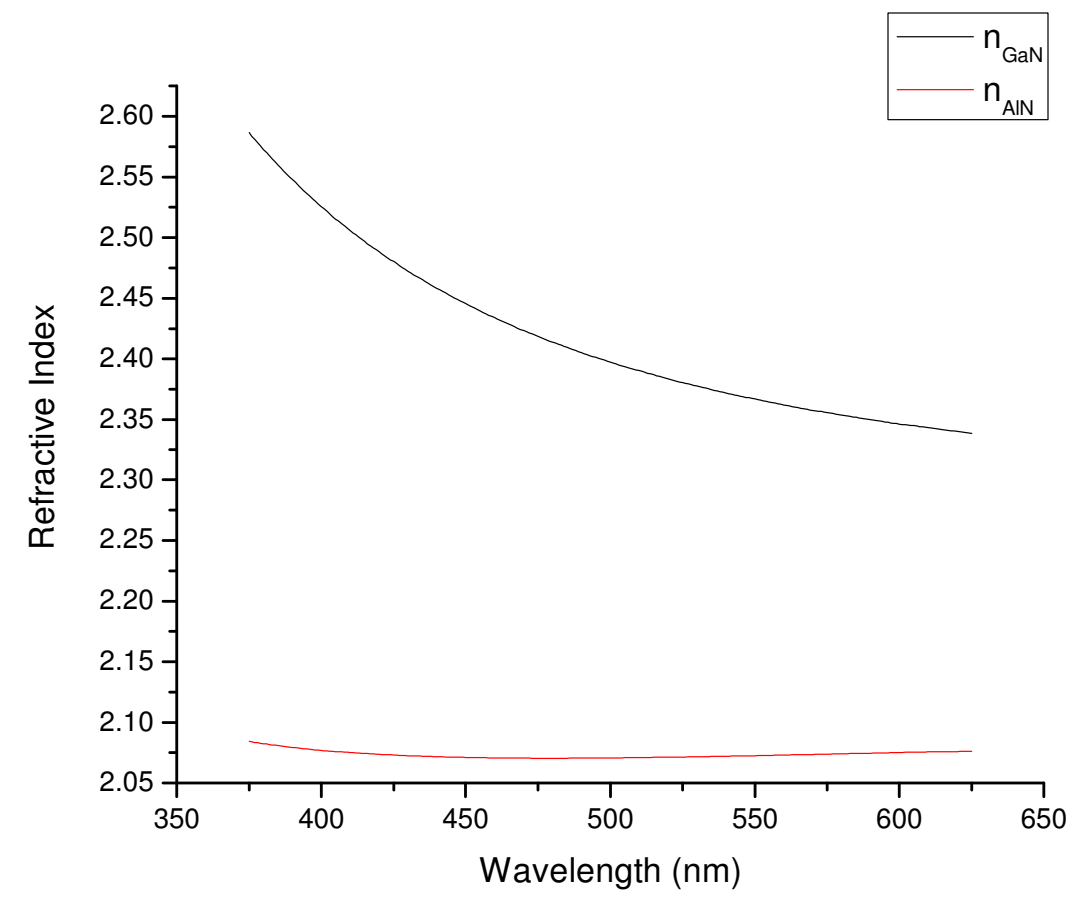

Figure 3.2.1 Refractive Indices of AlN and GaN

\subsubsection{Distributed Bragg Reflectors}

From these refractive indices, the quarter-wave thicknesses were calculated using equation 3.1. Finally, a loop containing the TMM calculations were carried out using the MATLAB code found in Appendix B. This simulation was repeated for the four target wavelengths of DBRs that are characterized in this work. Figure 3.2.2 shows the reflectivity for theoretical 6 period AlN/GaN DBRs at the four target wavelengths. As can be seen, the peak reflectivity increases at lower wavelengths. This is due to the refractive index contrast between AlN and GaN increasing, as shown in figure 3.2.3. As a result of this change in index contrast, a different number of bilayers are required to achieve a given reflectivity, depending on the target wavelength, as shown in figure 3.2.4. 


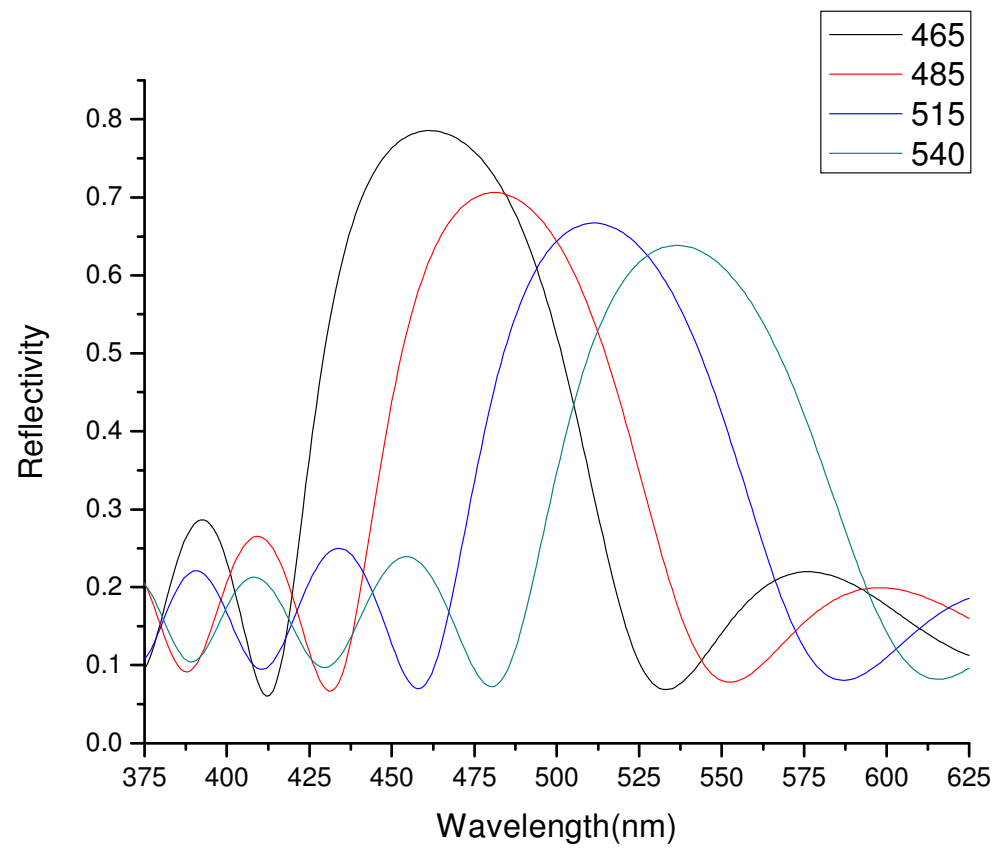

Figure 3.2.2 Reflectivity of Theoretical AlN/GaN DBRs

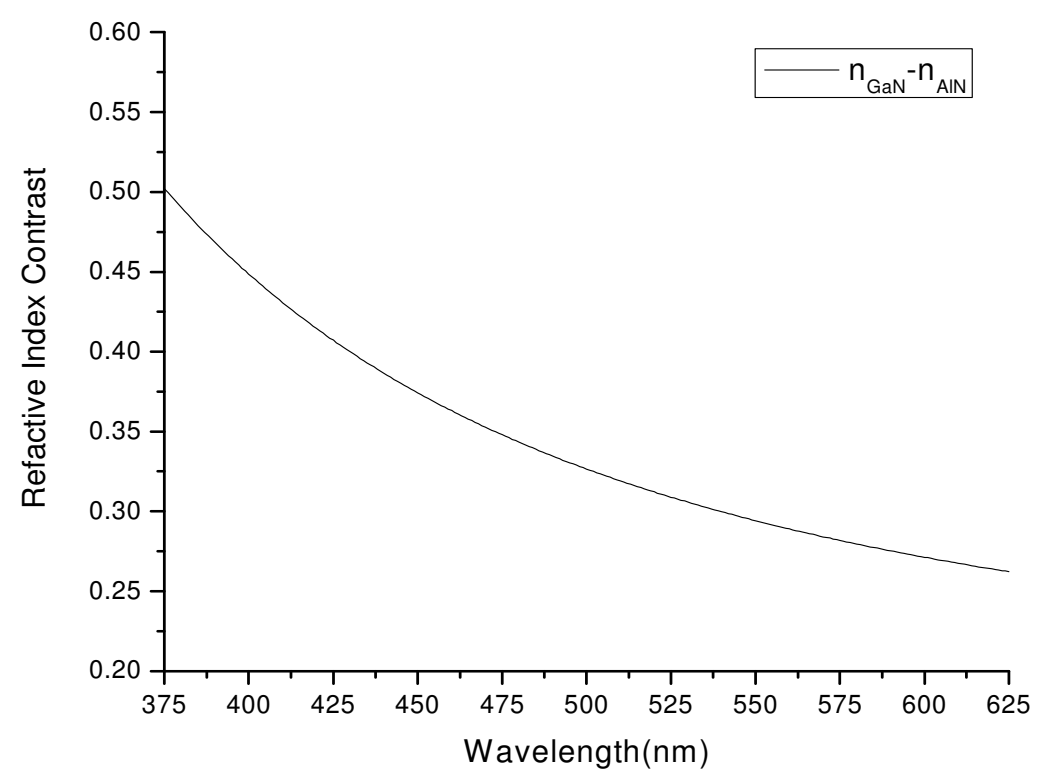

Figure 3.2.3 Refractive Index Contrast between AlN and GaN 


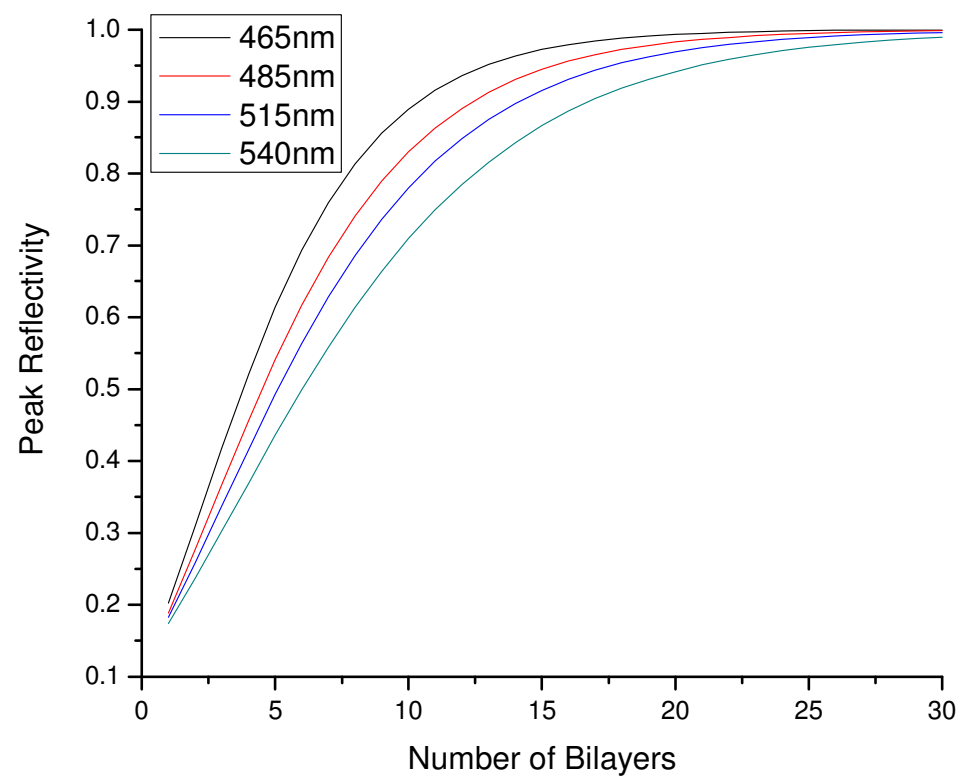

Figure 3.2.4 Peak Reflectivity vs. Number of Bilayers

\subsubsection{Additional DBR Simulations}

\subsubsection{Substrate Reflections}

In addition to simple DBR structures like those above, more complex structures and variations in structures were simulated using the Transmission Matrix Method in MATLAB. The first variation simulated was the effect of reflections from the bottom of the underlying GaN film that DBRs will be grown on. Figure 3.2.5 shows the reflectivity of a simulated 460nm 6 period AlN/GaN DBR on a 1600nm GaN substrate on bulk Sapphire, both with and without the reflections from GaN/Sapphire interface. This shows the effect of additional reflections that need to be accounted for during design. These reflections are fairly prominent for a 6 period DBR, but will die out as the number of bilayers is increased. 


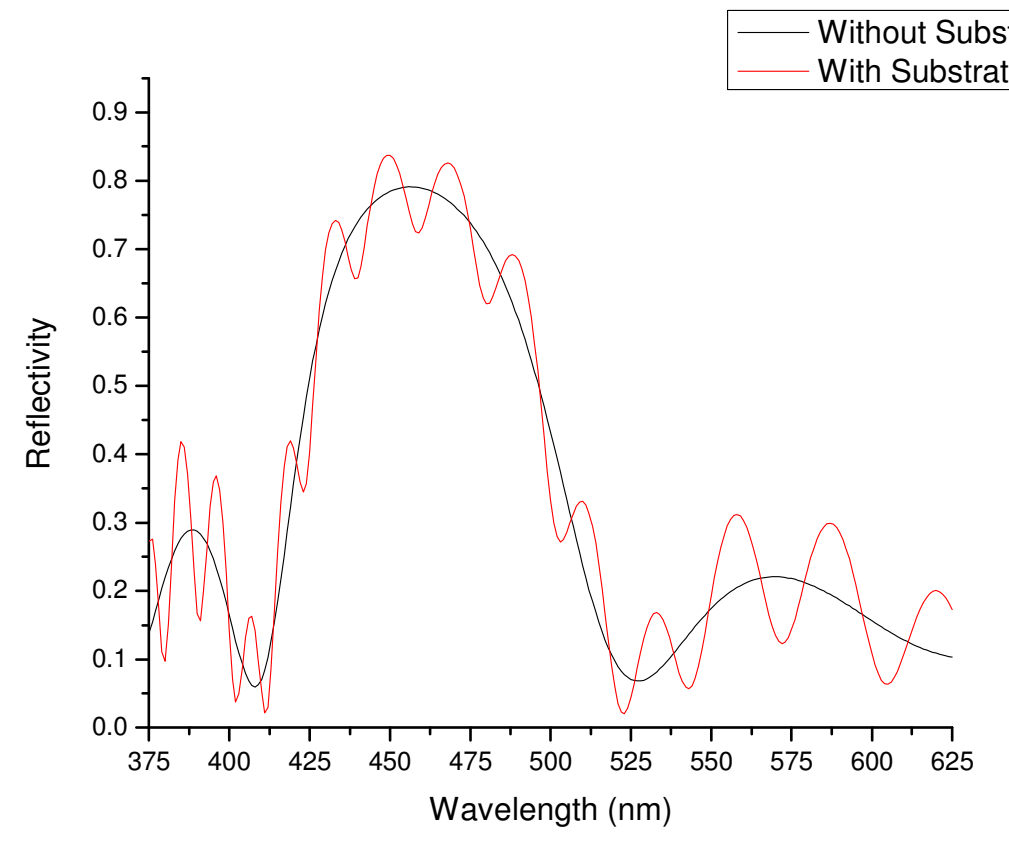

Figure 3.2.5 Effect of Substrate Reflections on Reflectivity

\subsubsection{Interlayers}

Interlayers in DBR structures are often added in an attempt to alter reflectivity or improve material quality. Modeling the effects of these interlayers is necessary to determine their reflectivity without the need for actual growths. An interlayer of variable thickness was simulated between a 6 period and 5.5 period AlN/GaN DBR. The interlayer was effectively created by varying the thickness of the GaN layer in the 6th bilayer from the substrate in a 12 period AlN/GaN DBR. Figure 3.2.6 shows the effects of two different interlayer thicknesses. For interlayers with even multiples of quarter-wave thickness, two peak DBRs are effectively created with a dip at the target wavelength. For odd multiples of quarter-wave thickness, a traditional DBR with higher secondary peaks is created. These structures can be ultimately tuned to acquire any desired reflection. 


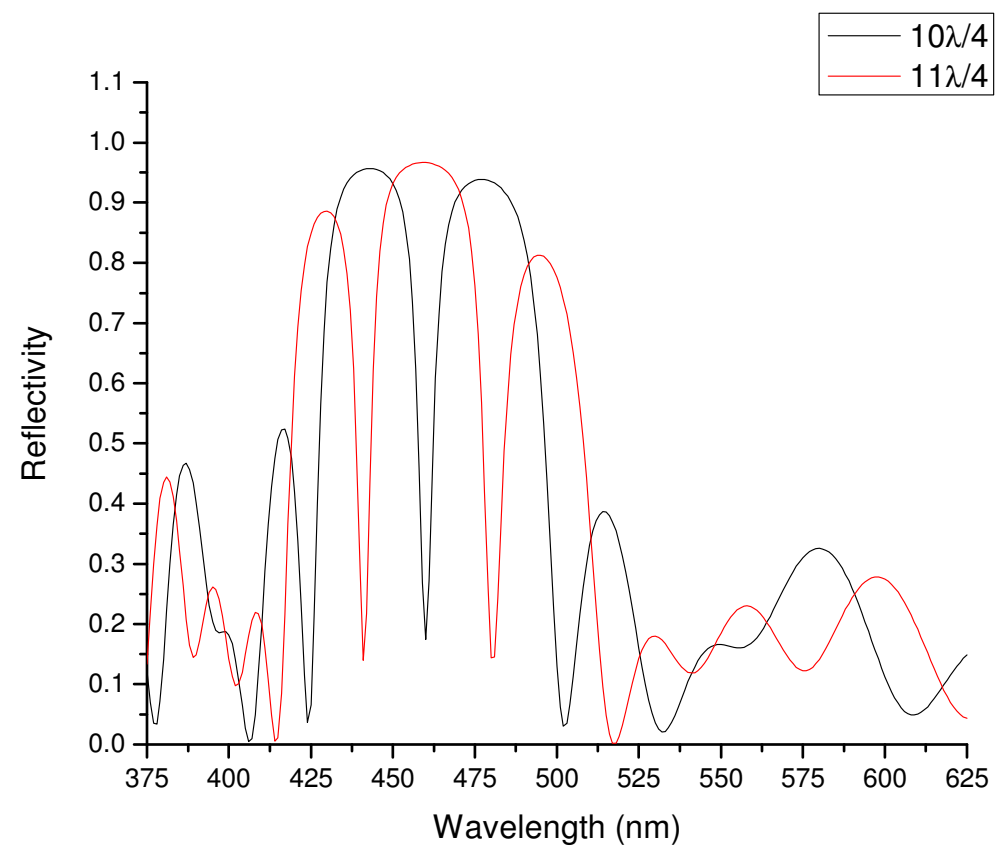

Figure 3.2.6 Reflectivity of DBRs with Interlayers

\subsubsection{Diffusion}

Diffusion is a common problem in DBR growths where the material transitions are not as abrupt as desired. Modeling this effect can lead to better predicting the reflectivity of structures. 6 period AlN/GaN DBR structures were simulated with varying degrees of diffusion. The AlN/GaN bilayers were change to become AlN/AlGaN/GaN layers with varying thicknesses of $\mathrm{AlGaN}$ to model the amount of diffusion. Figure 3.2.7 shows the result of these simulations. It can be seen that reflectivity decreases at an increasing rate as diffusion become more prominent. 


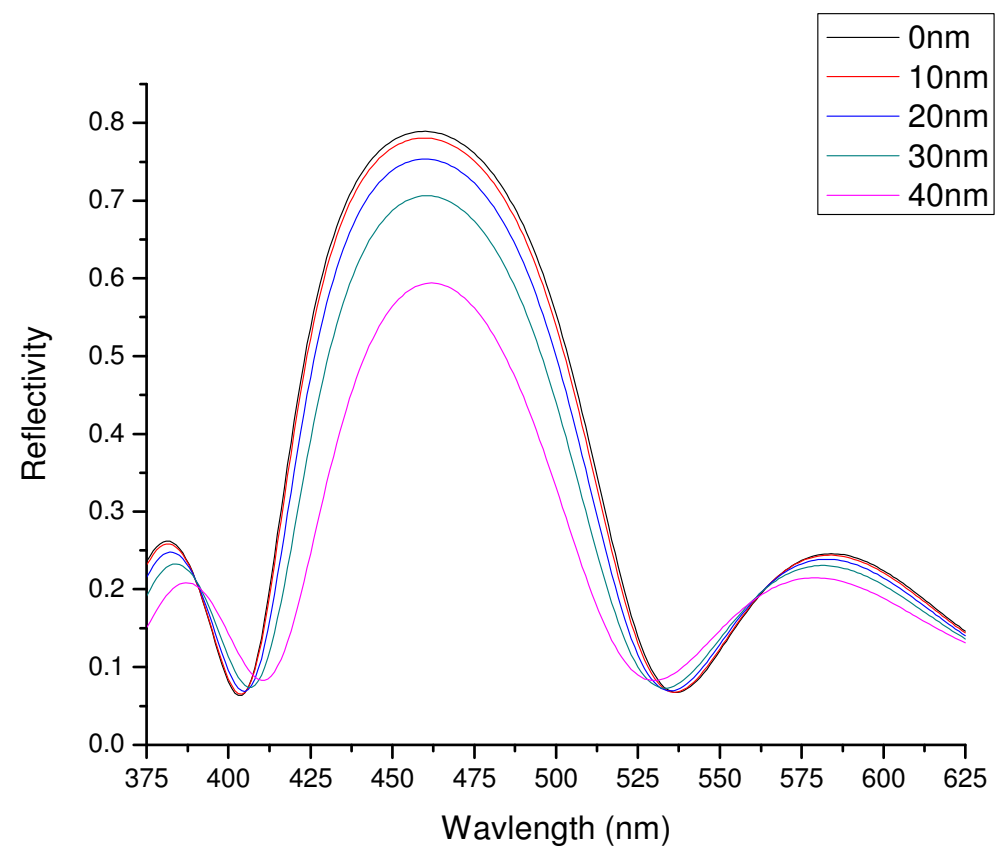

Figure 3.2.7 Effect of Diffusion on DBR Reflectivity

These MATLAB simulations will later be used to determine the quality of DBR growths and to explain their reflectivity. DBRs grown will be modeled and simulated using both their targeted values and the thicknesses and refractive indices determined using characterization techniques like ellipsometry. All simulations done using MATLAB have codes derived from that shown in Appendix B.

\subsection{MIT Electromagnetic Equation Propagation}

MIT Electromagnetic Equation Propagation (MEEP) is a finite-difference timedomain simulation software package that models electromagnetic systems [31]. The software is free under the GNU General Public License. The software uses Maxwell's equations and calculates them over time to determine results like transmission, reflection, and field patterns. 
Simulations with MEEP begin by creating 3-dimensional structures by defining their border coordinates and dielectric constants in a control (.ctl) file. Electromagnetic sources and desired output methods can then be set up in the control file. The control file is then invoked in a UNIX control window. Once run, the output can be plotted or displayed as an image, depending on type of output programmed.

The MEEP code in Appendix C was used to create a 540nm AlN/GaN structure. The code was set up to output both the reflectivity of the DBR structure as well as the structure and its field pattern. The output unique to this simulation method is shown in figure 3.3.1. The left half of the image is the structure created by the control file. This part of image is in grey-scale with darker areas indicating a higher dielectric constant or refractive index. The black area represents $\mathrm{GaN}$, grey represents $\mathrm{AlN}$, and white represents the background (air). The right half of the image is the field pattern in the structure. Blue represents positive amplitude and red represents negative amplitude light waves. The darkness of these colors represents the field strength. 


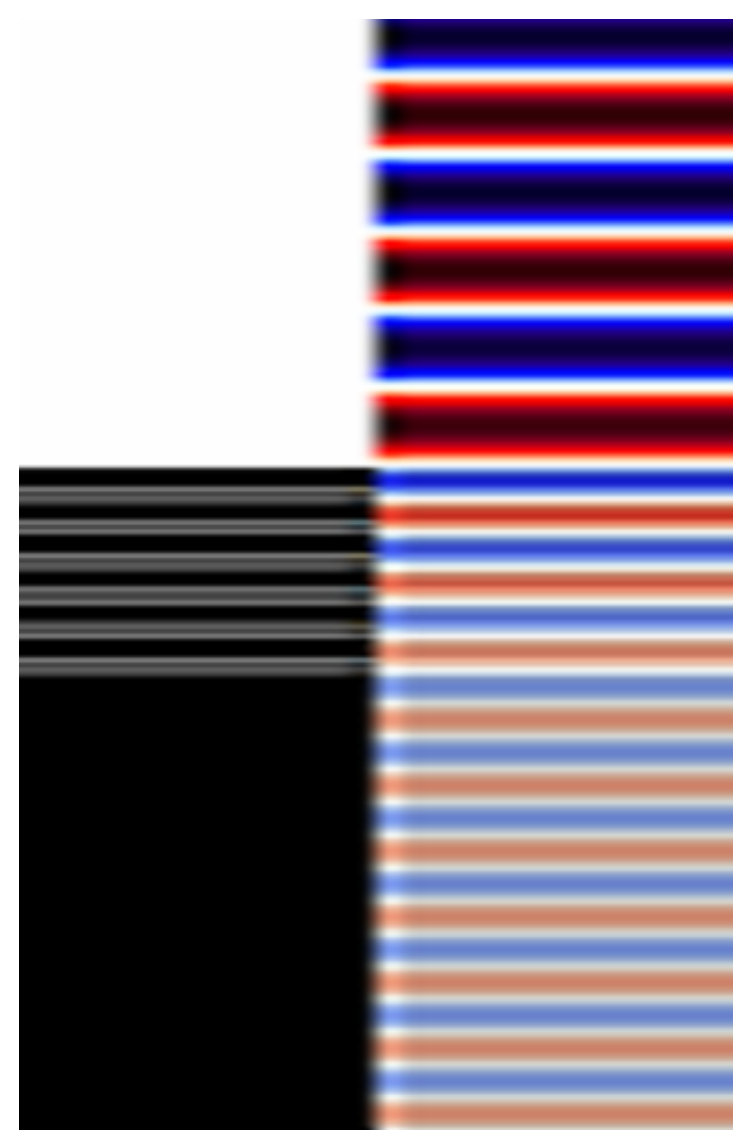

Figure 3.3.1 MEEP Output (DBR Structure and Field Pattern)

A few things can be gathered from this image. First, the wavelength can be seen to shorten in the material, due to the change in wavelength of light in different mediums. Also, it can be seen in the field pattern that there are zero crossings at the top and bottom of every bilayer, due to the quarter-wave thicknesses. It can be also seen that the field intensity decreases as it propagates through the DBR as a result of light being reflected. This output method is useful for understanding what is happening inside an optical system, something that cannot be accomplished in real situations.

The second type of output set up was the reflectivity, similar to that done using MATLAB. Figure 3.3.2 shows the output of this compared to the same structure simulated in MATLAB. As can be seen, the outputs are nearly identical, especially at lower wavelengths. The reason for the difference in output is not exactly known, but it is assumed to be a result of a slight difference in calculation methods and not especially critical. 


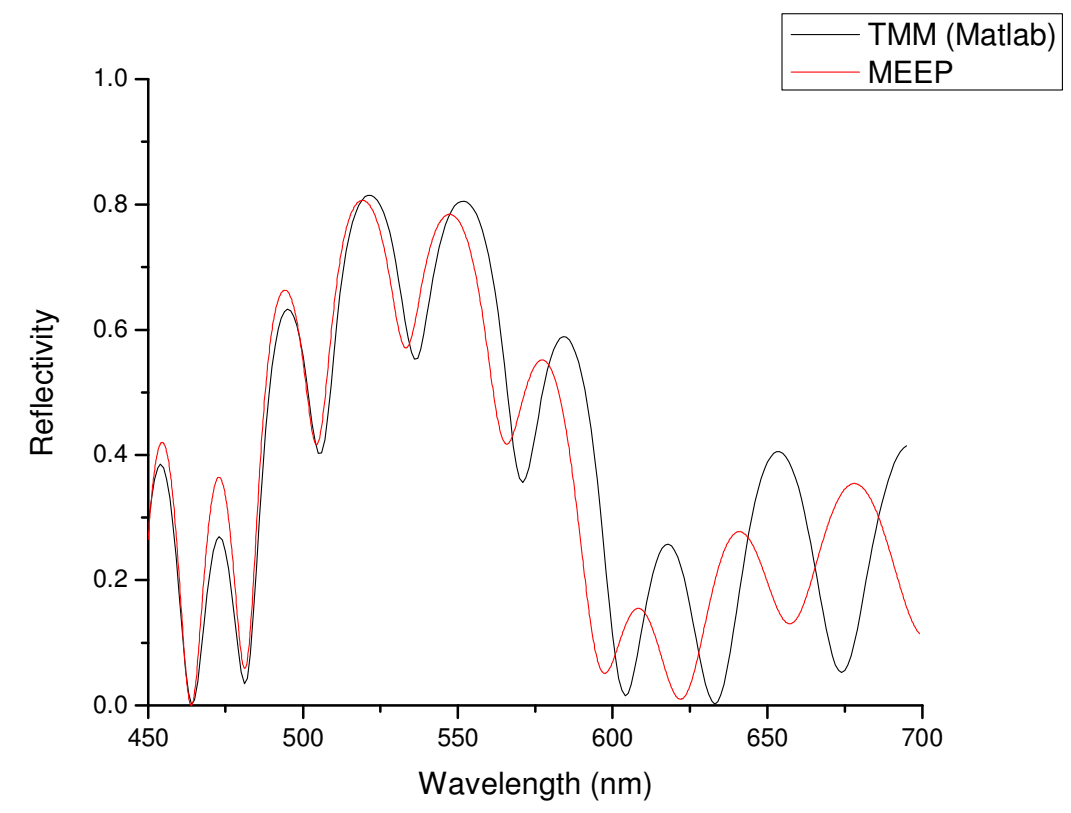

Figure 3.3.2 Reflectivity Comparison

MEEP has an advantage in that it can output field patterns that MATLAB cannot, but it has many disadvantages. First, the dielectric constants and refractive indices can only be entered as constants, not as a function of wavelength. Also, setting up the material system must be done using coordinates on a planned grid and is not easily edited. Because of these difficulties and the equivalent results provided by the both methods, only MATLAB simulations will be used in the remainder of this work. 


\section{Chapter 4 AIN/GaN Distributed Bragg Reflectors}

\subsection{Motivation}

III - N DBRs with high crystalline quality are critical components in applications like LEDs and VCSELs, but are one of the factors holding back their efficiency [32]. Being able to grow high quality III - N DBRs is critical to device performance and progression, which been held back by, among other factors, the inability to fabricated highly reflective, crack free DBRs [33]. Much work has been done on improving III - N DBRs by techniques such as using alloys, specifically AlGaN and AlInN, which provide less lattice mismatch to $\mathrm{GaN}$ [34]. There has also been work to improve films by adding additional layers such as interlayers, buffer layers, and superlattices [18, 35, 36]. While these techniques have had degrees of success, work still needs to be done to improve AlN/GaN DBRs without the need for alloying, which can decrease index contrast, or adding additional material layers, which increases production cost and time as well as results in reflectivity variations.

In this chapter, AlN/GaN DBRs will be grown, characterized, and simulated in order to optimize and improve growths without the need for alloying or additional layers. The effect of growth parameters on refractive index, film quality, and DBR reflectivity will be investigated. Efforts will be made to reduce strain and cracking and to improve overall crystalline quality.

\subsection{AIN/GaN DBR Growth}

AlN/GaN DBRs in this work were grown via MOVPE in an AIXTRON 200/4 RF-S horizontal reactor. Trimethylgallium (TMGa), Trimethylaluminum (TMAl), Trimethylindium (TMIn), and ammonia (NH3) are used as the precursors for Gallium, Aluminum, Indium, and Nitrogen, respectively. A $2 \mu \mathrm{m}$ thick n-type GaN epitaxial layer was first grown on a bulk (0001) sapphire substrate at $980^{\circ} \mathrm{C}$. Alternating AlN/GaN layers 
were then grown with temperatures of $1000^{\circ}$ and $980^{\circ} \mathrm{C}$, respectively. The V/III ratio for the AlN and GaN layers were 1000 and 700, respectively. For some AlN layers, TMIn was introduced into the reactor as surfactant and the ratio of the TMIn to TMAl flow was varied from 0 to 0.6 in an attempt to reduce strain [37]. Figure 4.2.1 is an example SEM image of a 6 period AIN/GaN DBR grown using MOVPE. The lighter grey areas are the n-type GaN base layer and $6 \mathrm{GaN}$ layers for the DBR while the darker areas are the $6 \mathrm{AlN}$ layers for the DBR.

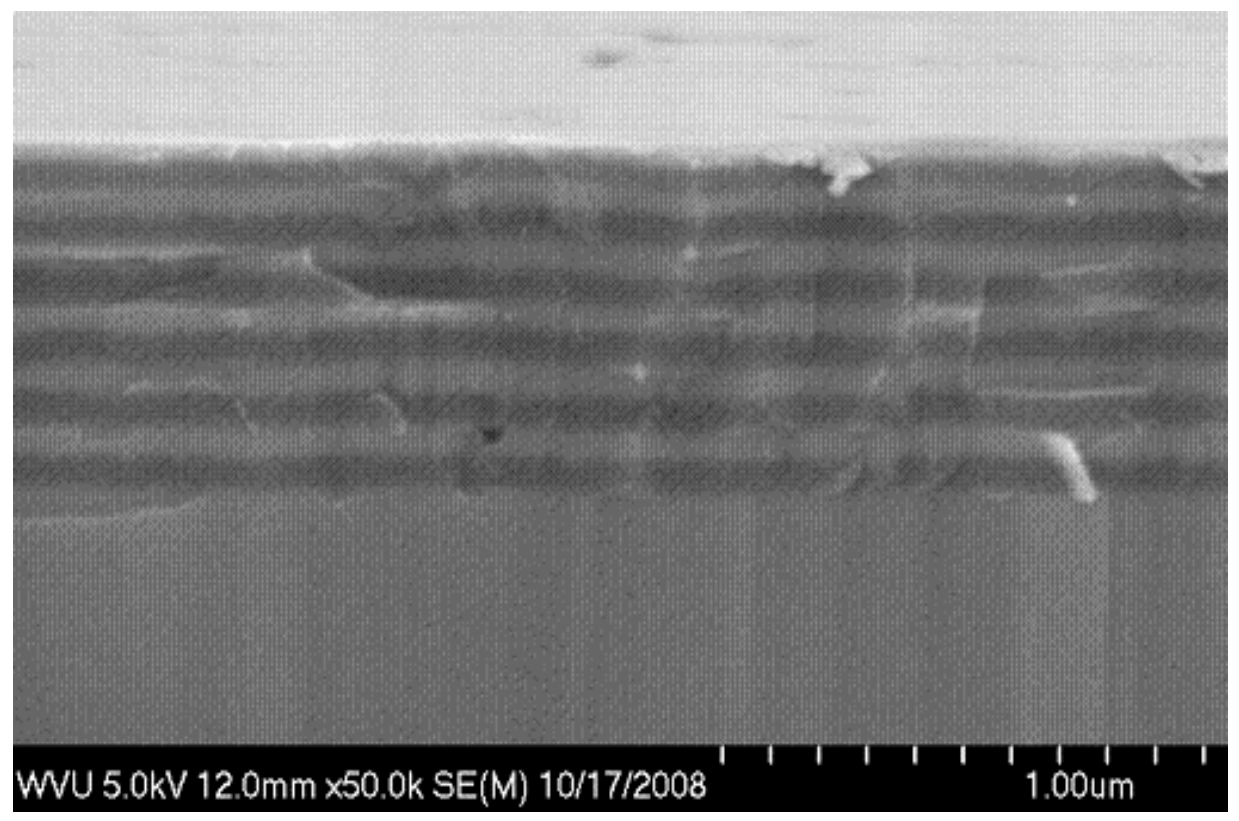

Figure 4.2.1 SEM Image of a 6 Period AlN/GaN DBR

\subsection{AIN/GaN DBR Characterization}

AlN/GaN DBRs were characterized and simulated in order to compare them to theoretical values. Figure 4.3.1 shows the normal reflectivity of DBRs grown at four target wavelengths found using spectroscopy. An aluminum coated Pyrex mirror was used as a background to normalize the reflectivity. When compared to figure 3.2.2, it can be seen that 
the reflectivity of these DBRs are lower than the theoretical data, especially for those at lower target wavelengths.

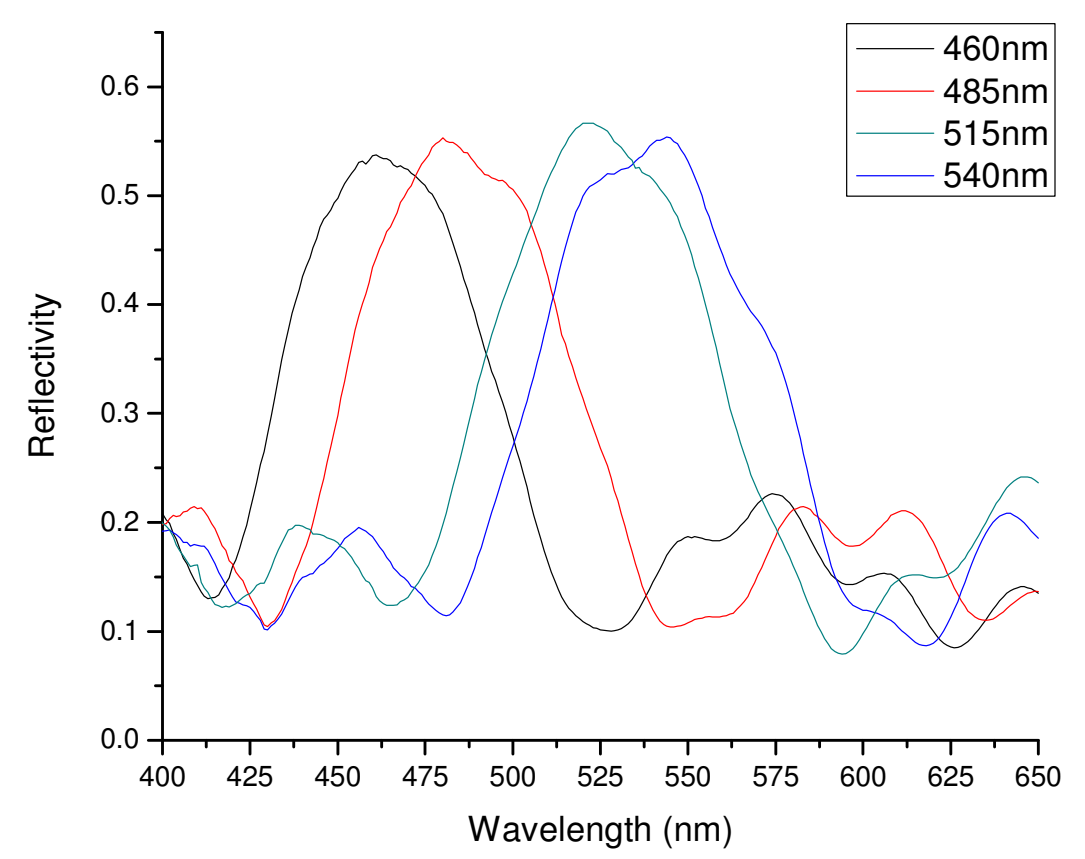

Figure 4.3.1 Reflectivity of AlN/GaN DBRs

The reflectivity was found to vary little according on wavelength. Figure 4.3.2 compares the peak reflectivity of these DBRs with those predicted by literature values for refractive index. As can be seen, the peak reflectivity is much closer to that expected for higher target wavelengths. This could be an indication that the index contrast of our growths does not vary as much as predicted in literature. The reduced reflectivity could also be due increased cracking and strain for the thinner layers. This would agree with literature that suggests that cracking can be dependant on film thicknesses and an increase in cracking can lead to a decrease in reflectivity $[18,38]$. The exact reason for this will be investigated in the following chapter. 


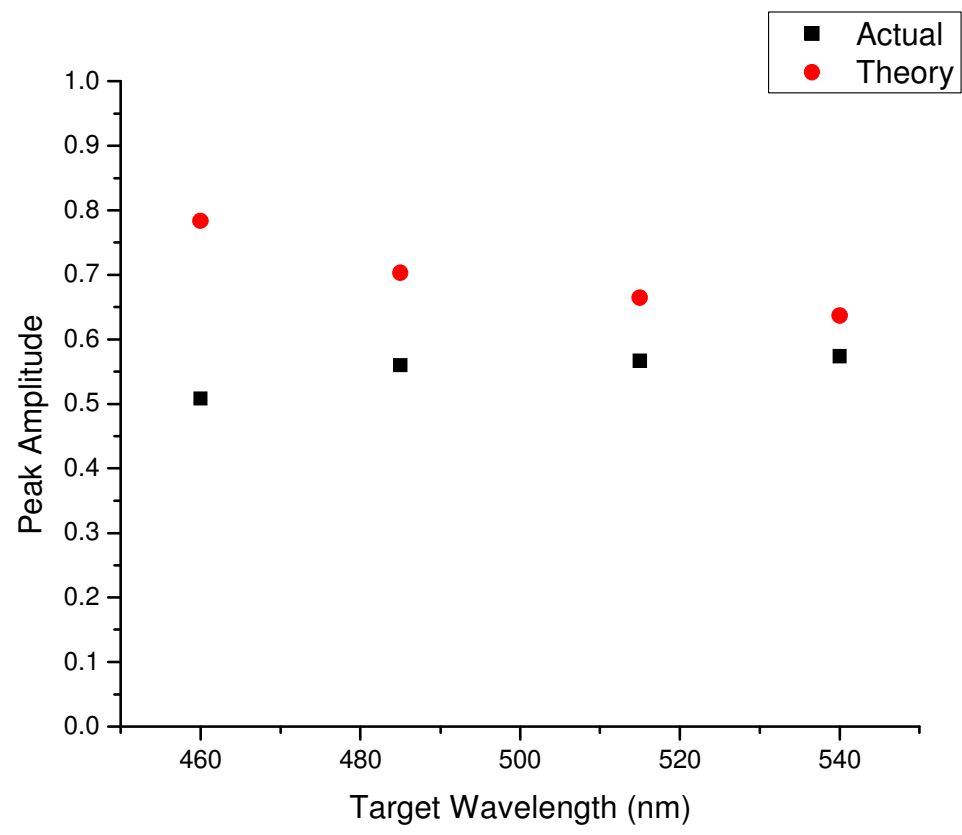

Figure 4.3.2 DBR Peak Reflectivity Compared to Theoretical

In addition to reflectivity measurements, ellipsometry was performed on the samples. From the ellipsometry data, models were fit to the data to determine film thicknesses and refractive indices. The models were performed both by having fixed refractive indices and variable thicknesses and by having both values variable. Figure 4.3.3 shows values for the normalized refractive indices obtained using this method for a DBR with a target wavelength of 540nm. Figures 4.3.5 and 4.3.6 show the normalized thicknesses obtained from this method and using SEM imaging for the same sample. The thicknesses determined using ellipsometry were found to be reasonable when compared to SEM values. The values were normalized using values expected from literature and growth parameters. While these values were modeled using many variables and are probably not exact, they serve as a good baseline for approximating layer properties. In figure 4.3.3, it can be seen that the refractive indices for all layers were found to be above those predicted in literature. These elevated refractive indices were verified by performing simulations with fixed thicknesses set by growth targets as well as SEM values. These elevated refractive 
indices may again be a result of stress and cracking as it has been reported that changes in refractive index can result from changes in strain [39].

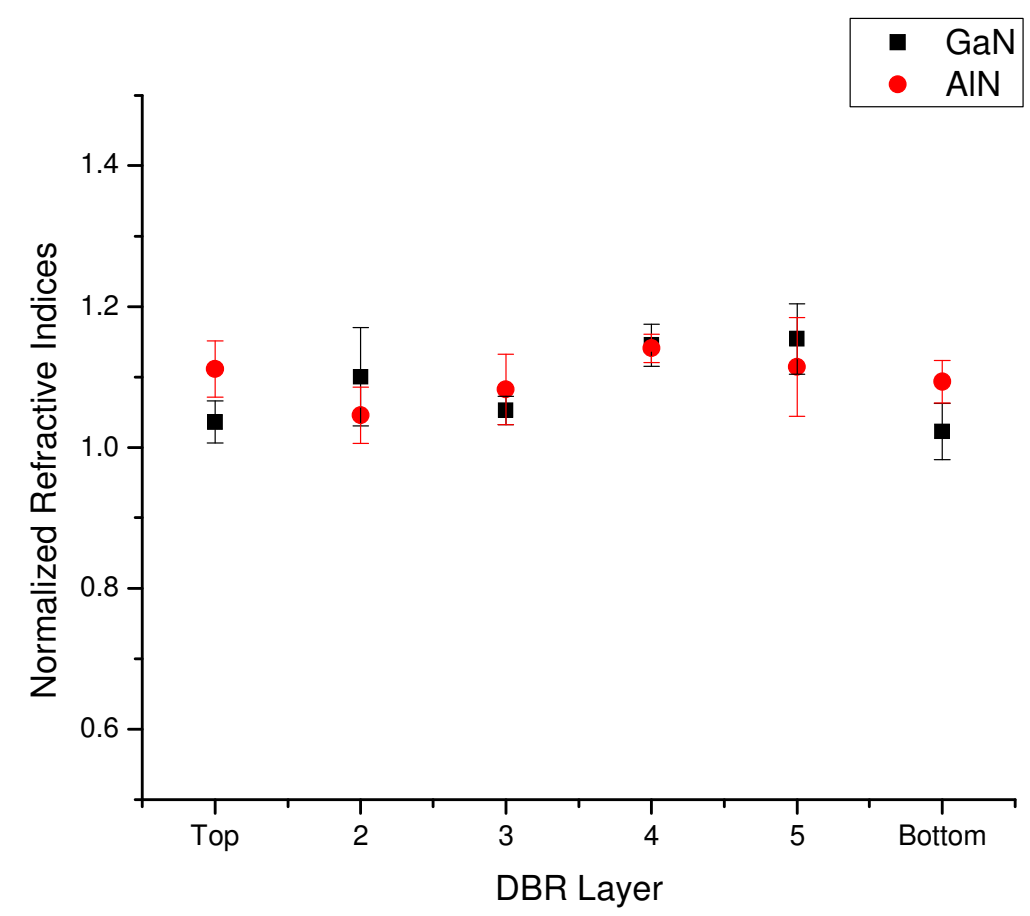

Figure 4.3.3 Normalized Refractive Indices for a 540nm DBR 


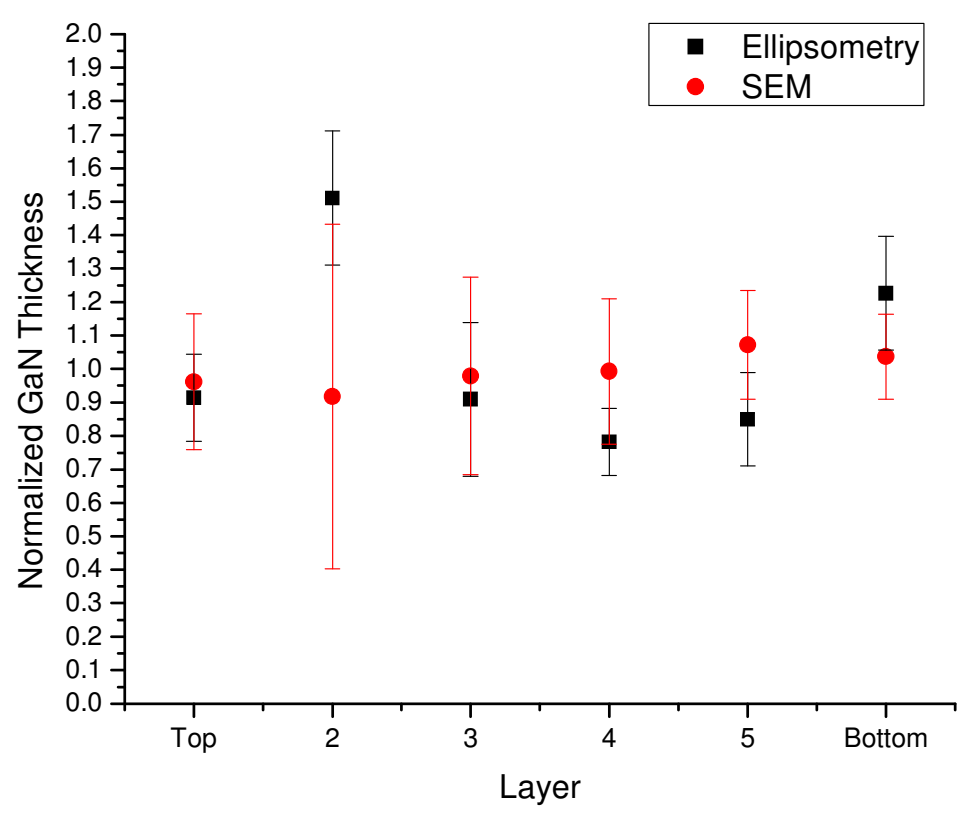

Figure 4.3.4 Normalized GaN Thicknesses for a 540nm DBR

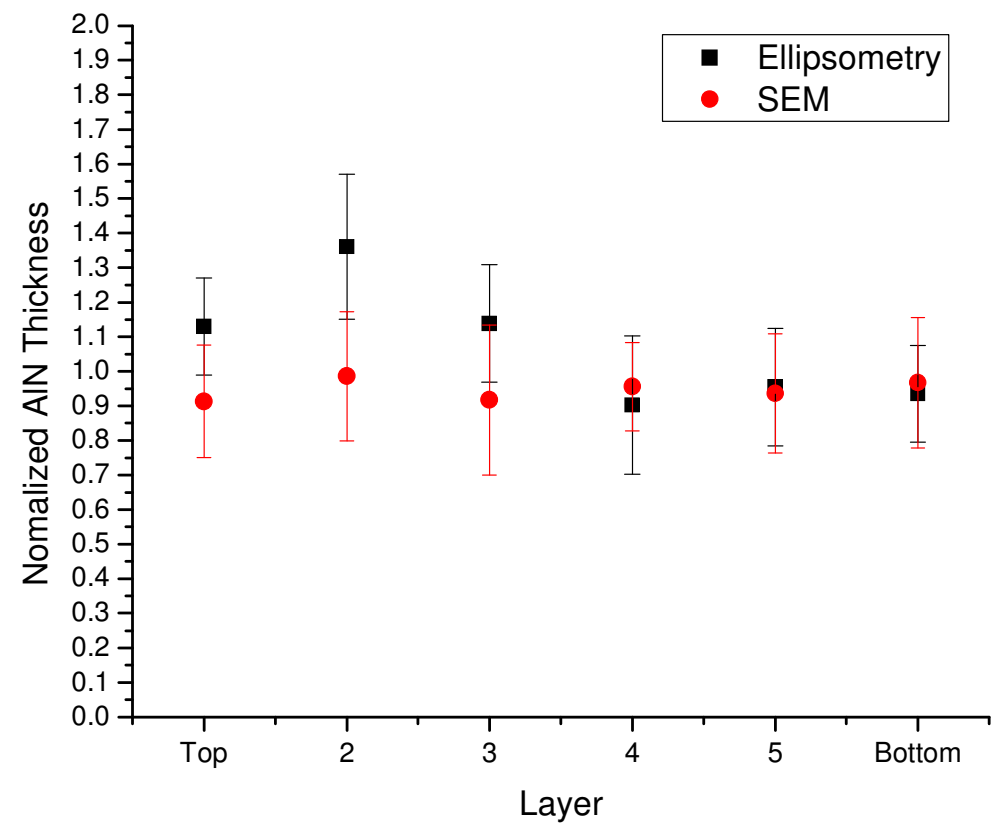

Figure 4.3.5 Normalized AlN Thicknesses for a 540nm DBR 
From the modeled values acquired, the transmission matrix method via MATLAB was used to recreate the reflectivity that would be associated with the modeled structure. Figures 4.3.6 and 4.3.7 show the result of simulations done with both fixed and variable refractive indices for $515 \mathrm{~nm}$ and $540 \mathrm{~nm}$ DBRs. The data suggests that having all values variable is best for recreating the actual reflectivity as this method fits very well with the reflectivity acquired using spectroscopy. The bumps in the reflectivity were found to be a result of interference peaks attributed to the substrate GaN layer and should be expected, especially for a DBR with only 6 periods.

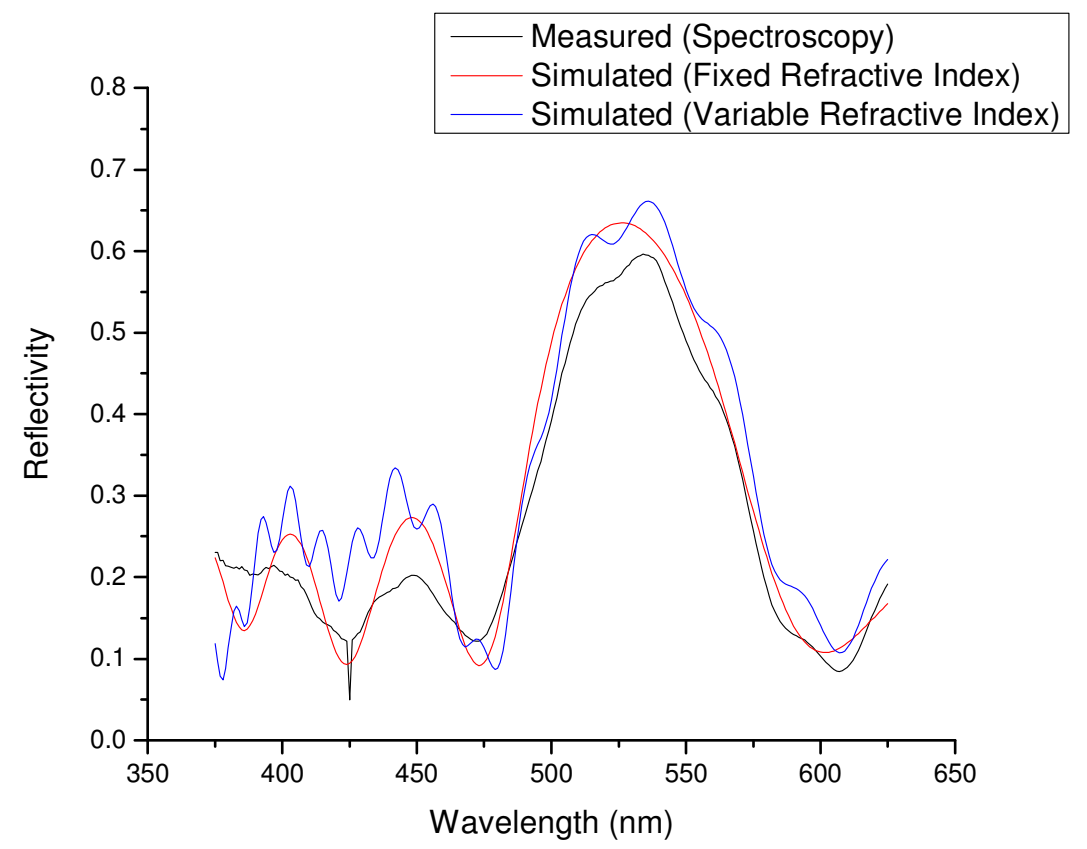

Figure 4.3.6 Comparison of Simulated and Measured Reflectivity for a 540nm DBR 


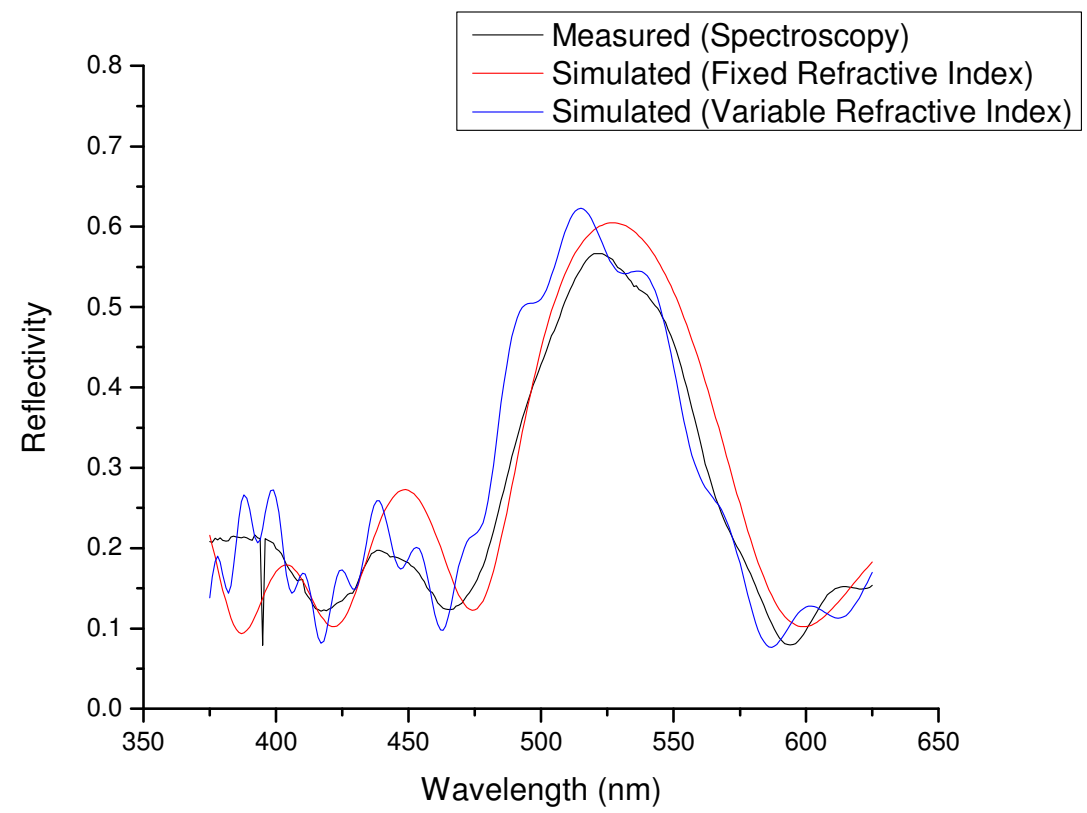

Figure 4.3.7 Comparison of Simulated and Measured Reflectivity for a 515nm DBR

This method was found to be very successful for DBRs with target wavelengths above approximately $480 \mathrm{~nm}$, but was more limited for those with lower target wavelengths where models became less reliable. This was a result of the light source for the ellipsometer not having sufficient irradiance at wavelengths low enough to completely model lower wavelength DBRs. For this reason, refractive index values used in this work are exclusively from DBRs with target wavelengths above $480 \mathrm{~nm}$.

\subsection{AIN/GaN DBR Refractive Indices}

Using ellipsometry, it was found that the apparent refractive indices for DBRs grown in this work were much higher than those predicted by literature. Figure 4.4.1 shows the average refractive indices for all DBR samples grown at the four target wavelengths. It can be seen that the apparent refractive indices for all of the target wavelengths are higher than that predicted by literature. A similar result was also found for AIN layers. 


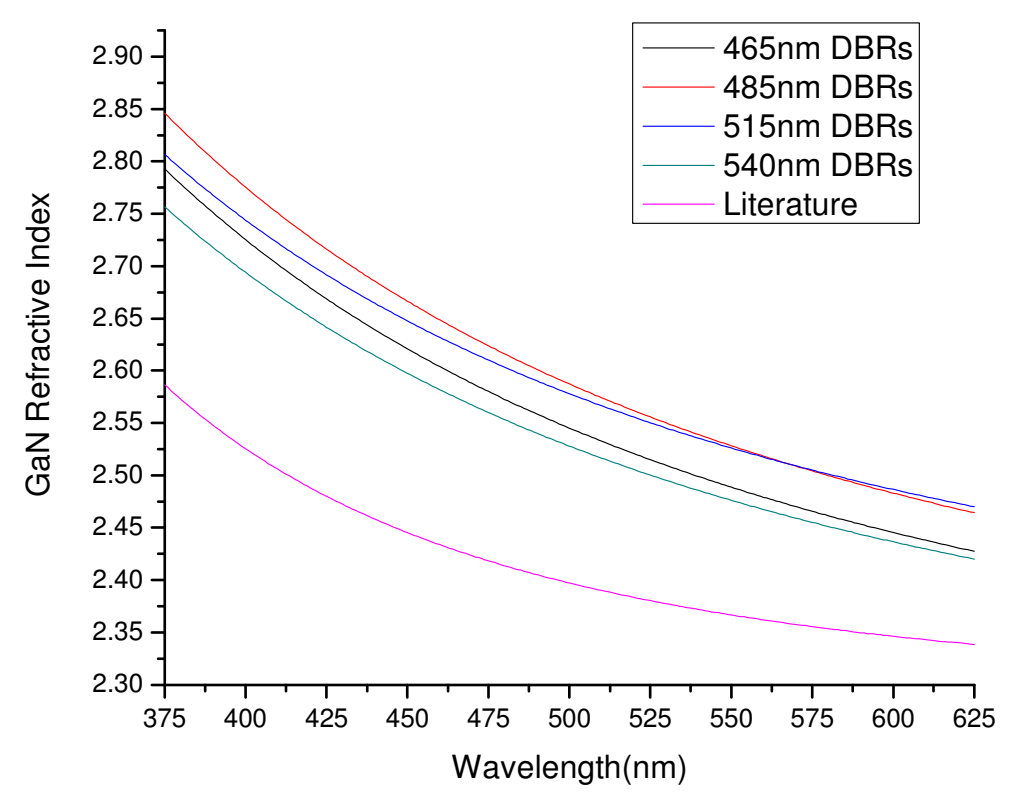

Figure 4.4.1 DBR Refractive Indices Compared to Literature

In order to ensure that these higher indices were not just a result of growth conditions; several GaN, $\mathrm{nGaN}$, and $\mathrm{AlN}$ thin films grown under the similar conditions were characterized as well. The DBR refractive indices compared to both literature and single films are shown in figures 4.4.2 (GaN) and 4.4.3 (AlN). It can be seen that for both $\mathrm{AIN}$ and GaN, the DBR refractive indices are significantly higher than those predicted by literature and those found for single films grown under similar conditions. This indicates that the high apparent refractive indices were a result of something other than growth conditions. The reason for these higher refractive indices will be investigated in the following chapter In addition, the refractive index contrast between the two materials, shown in figure 4.4.4, is not as highly wavelength dependent as predicted by literature. This at least partially explains why the DBRs peak reflectivity seems to vary much less with wavelength than would be expected. 


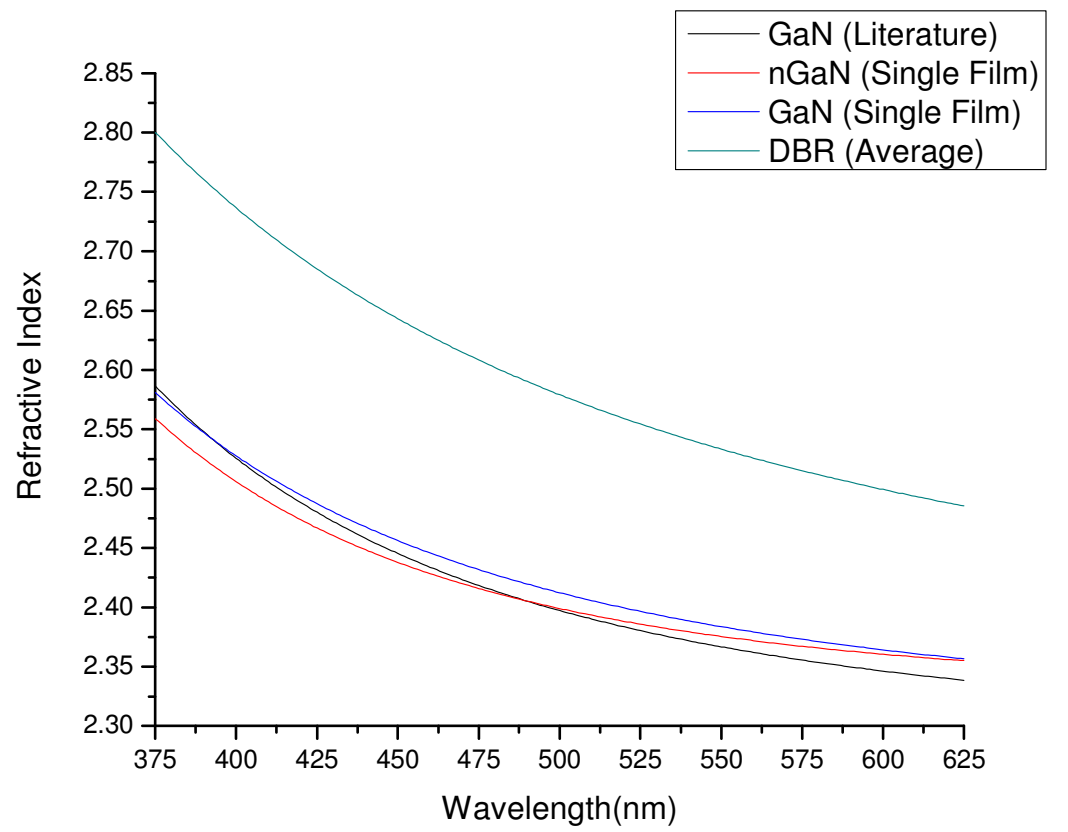

Figure 4.4.2 GaN Refractive Index Comparison

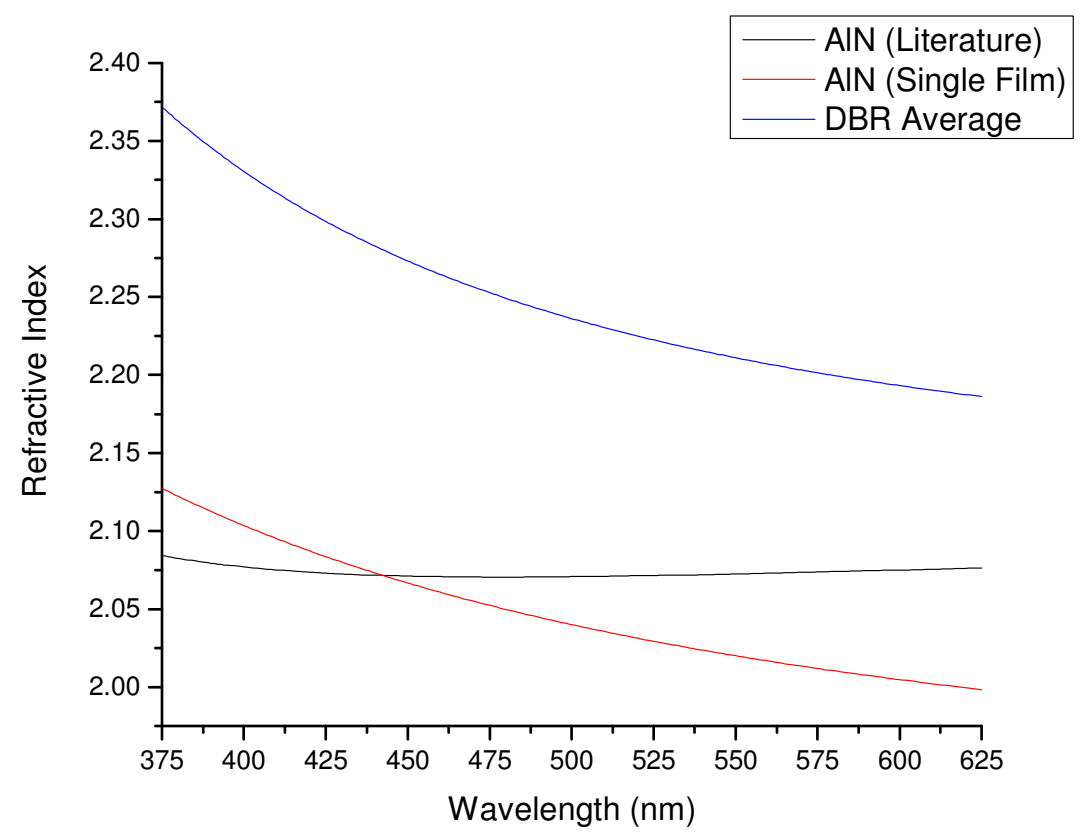

Figure 4.4.3 AlN Refractive Index Comparison 


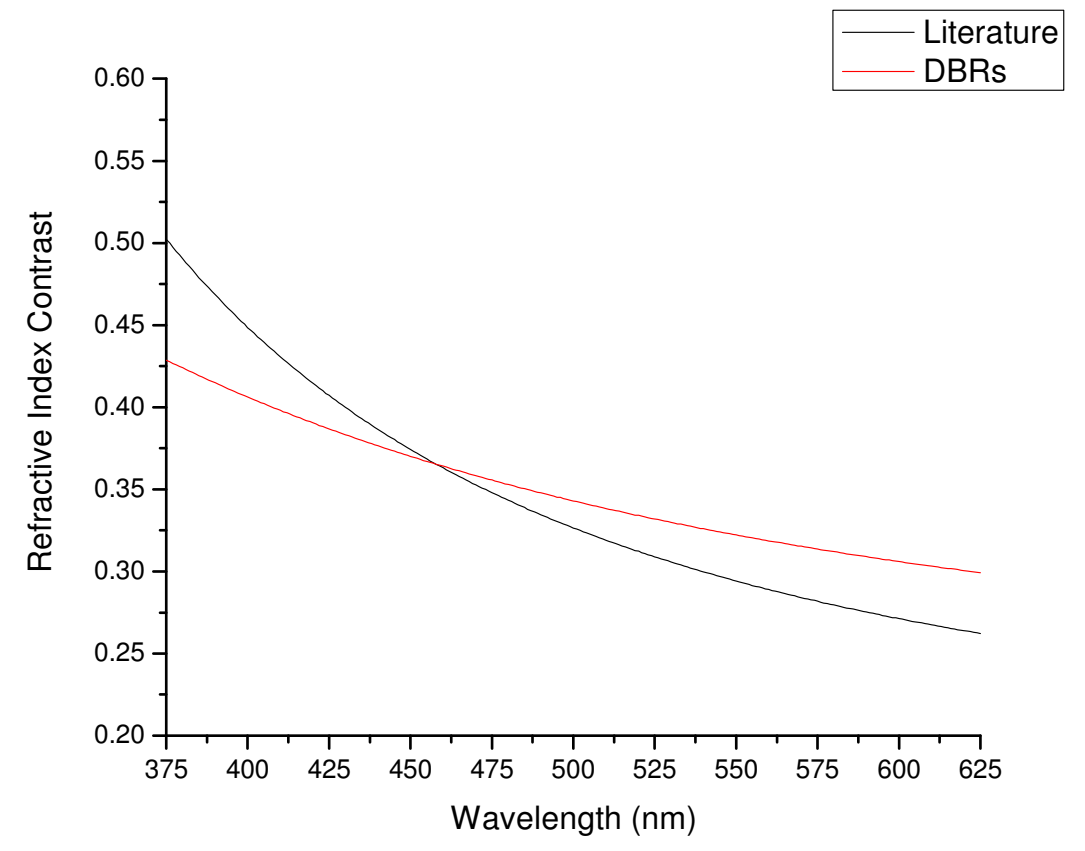

Figure 4.4.4 Refractive Index Contrast for DBRs and Literature 


\section{Chapter 5 Effect of Strain on AIN/GaN Distributed Bragg Reflectors}

The AIN and GaN DBR bilayers characterized in this work were found to have higher apparent refractive indices than those stated by literature and found for single films grown under identical growth conditions. It was also found that the reflectivity for DBRs, especially those at lower target wavelengths, was lower than that expected. Establishing the reason for this is integral to understanding and improving the quality and reflectivity of DBRs. One possible reason for the increased apparent refractive indices is strain resulting from the lattice mismatch between the relatively thin layers of the DBRs. This strain would not be found in single films and would account for the difference in refractive indices.

It has been reported that an increase in refractive index in III - Ns and other materials like sapphire can be attributed to an increase of tensile strain [39, 40]. Other groups have found that tensile strain in the AlN and AlGaN layers of III - N DBRs has resulted from lattice mismatch and is a common problem in III - N DBR structures [18]. This strain has been known to lead to film cracking, which can seriously affect film properties [41]. Establishing a connection between strain and apparent refractive index and using this information to improve growths to limit strain will be discussed.

\subsection{Strain and Cracking in AIN/GaN DBRs}

Lattice mismatch between GaN and AlN have been shown to cause tensile strain in AlN layers which in return can result in the formation and propagation of cracks in AlN/GaN DBR structures [42]. This cracking has been shown to significantly decrease the reflectivity of DBRs [18]. Characterizing and reducing this cracking is necessary for improving DBR performance. AlN/GaN DBRs grown in this work were found to exhibit cracking in a manner consistent with results found by other groups. Figure 5.1.1 is an optical microscope image of the top of an AlN/GaN DBR structure showing this cracking. 
This cracking leads to domains or distinct separated areas of good material separated by cracked areas of poorer material [38].

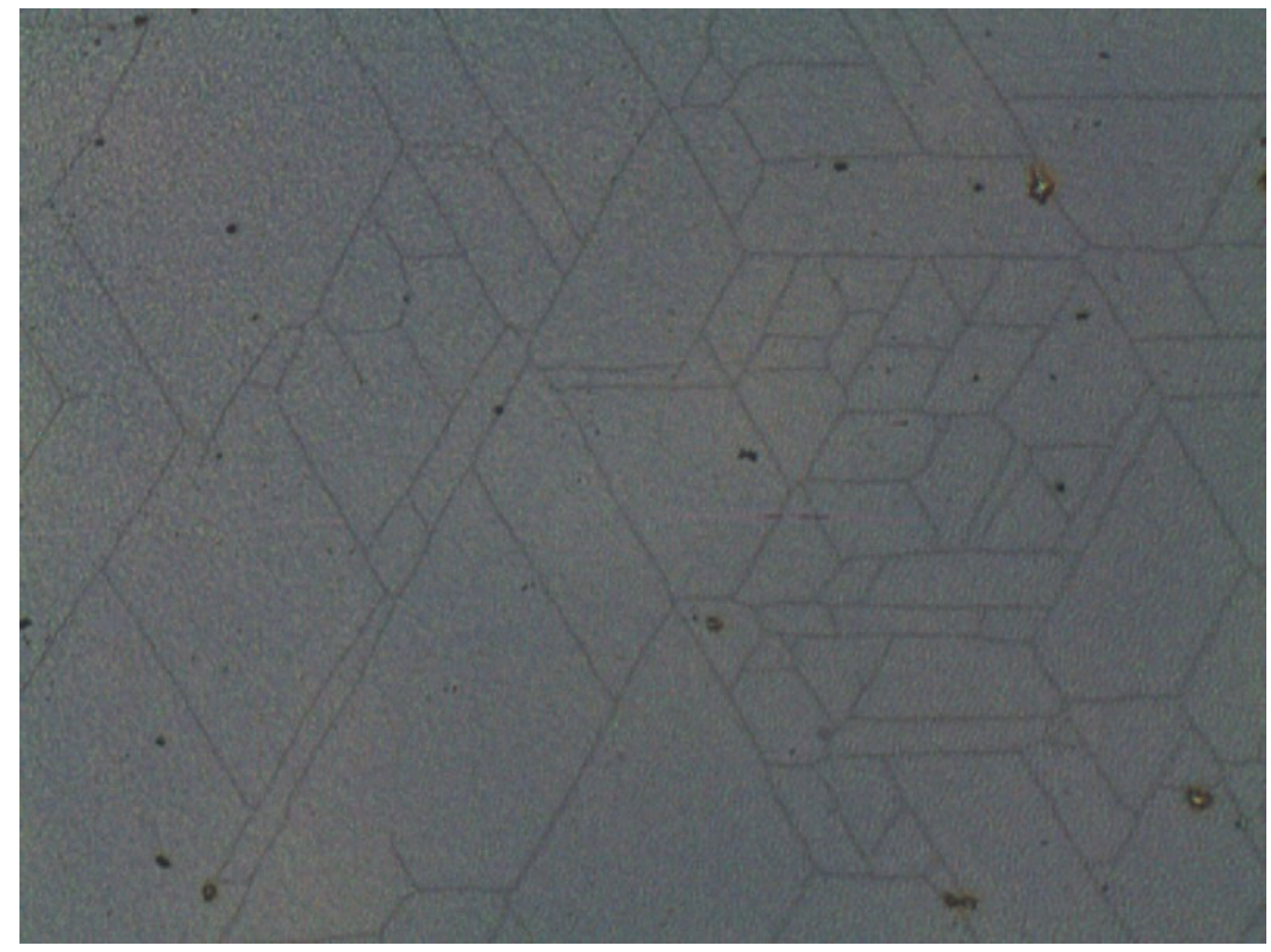

Figure 5.1.1 Optical Microscope Image of a 6 Period AlN/GaN DBR

This type of cracking in AlN/GaN DBRs has been reported to decrease the reflectivity of DBRs, which was attributed to scattering, diffraction, and absorption due to the poorer material [43]. Reflectivity measurements performed on the sample imaged above were done using spectroscopy and compared to the theoretical. In figure 5.1.2, it can be seen that there is a great reduction in reflectivity compared to what is expected. This is attributed to the strain and subsequent cracks in the DBR structures. This also explains the higher apparent refractive indices found for the DBR structure. The apparent refractive indices found are thought to be related to the percentages of good and poor material in the 
DBRs and therefore dependant on the amount of cracking. For this reason, the reflectivity of the DBRs and apparent refractive indices should be proportional to the amount of cracks found in the samples. We will attempt to find correlation between the amount of cracking and the resulting refractive indices and reflectivity.

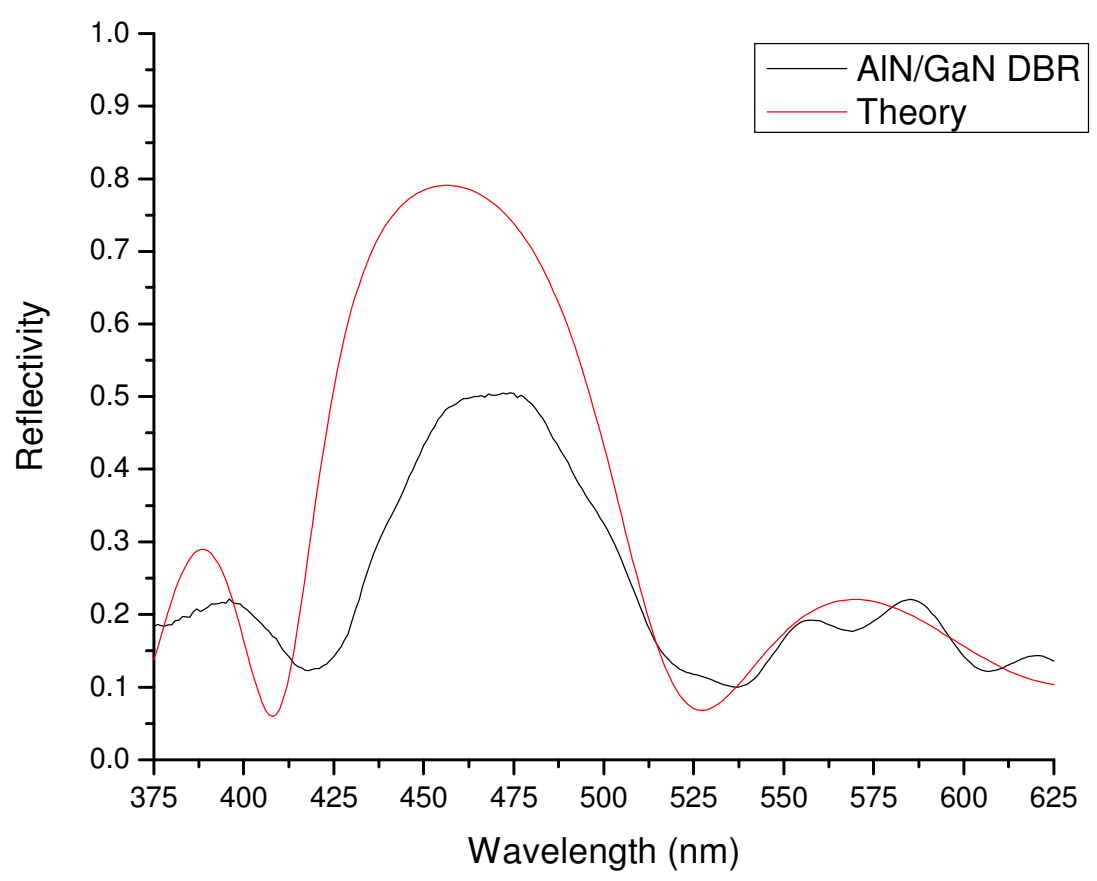

Figure 5.1.2 Reflectivity of AlN/GaN DBR Compared to Theoretical

\subsection{Crack Suppression}

Suppressing cracking in AlN/GaN DBRs is necessary to improve material quality and performance. Achieving smooth surfaces is also necessary for applications where devices will be grown on top of the DBR structure [18]. For this reason, many groups have worked on suppressing cracks using a variety of methods like alloying and using additional material layers. One method attempted by others in this group is using Indium as a 
surfactant during the growth of AlN layers [37]. The work focuses on using Indium to relieve the strain in the AlN layers in order to inhibit the creation and propagation of cracks. The Indium is thought to occupy defect areas and absorb strain, thus inhibiting cracking and improving material quality [37, 44]. In the work, TMIn was introduced into the reactor as surfactant and the ratio of the TMIn to TMAl flow was varied from 0 to 0.6 in an attempt to reduce strain. It was found that the amount of cracking was affected by the ratio of TMIn to TMAl flow. The amount of cracking was quantified by the density of domains (distinct areas separated by cracks). Figure 5.2.1 shows the results. It can be seen that the domain density does vary with the TMIn/TMAl ratio as proposed. Samples with different TMIn/TMAl flow rates will be investigated to determine their apparent refractive indices and reflectivity.

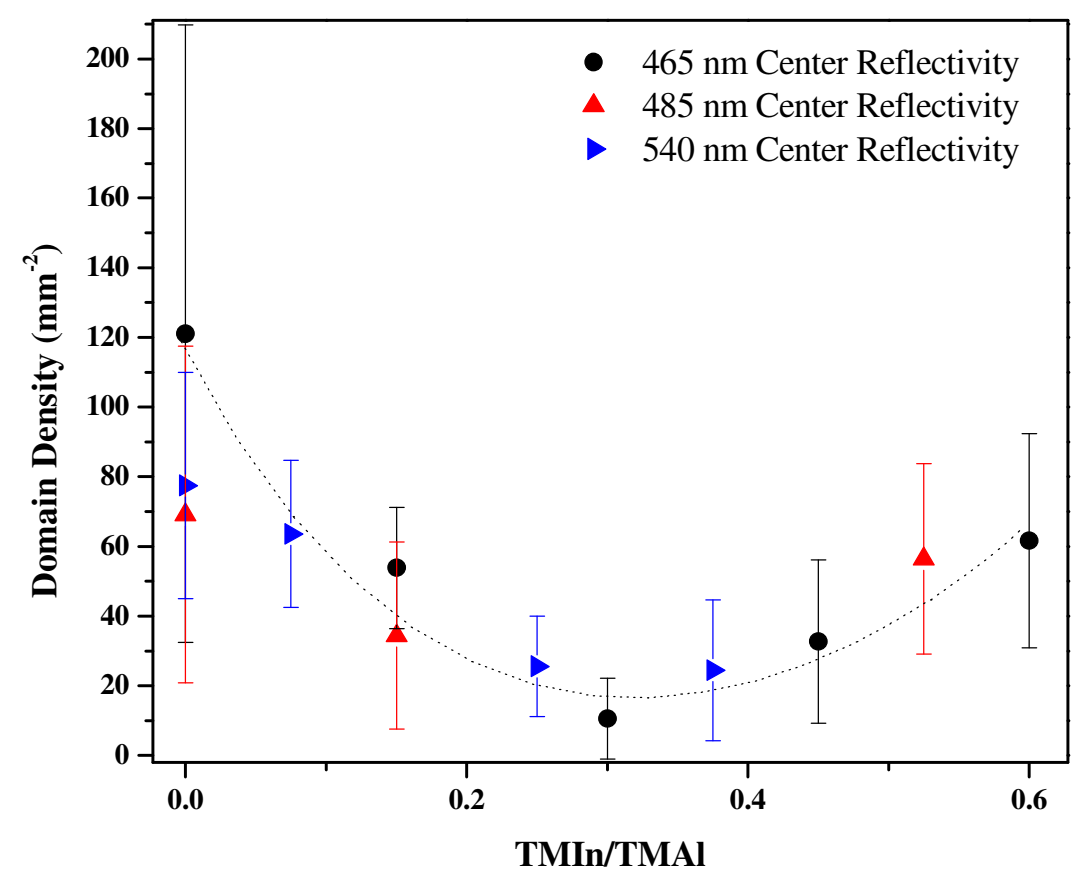

Figure 5.2.1 Effect of TMIn/TMAl ratio on Domain Density [37] 


\subsection{Effect of Strain on Refractive Index and DBR Reflectivity}

If the increased apparent refractive indices found for the AlN/GaN DBRs are a result of the strain and the subsequent cracking in the films, the refractive indices should also vary with the TMIn/TMAl ratio and, therefore, the resulting domain density. In order to test this theory, the refractive indices were found for the GaN and AlN layers for all 6period DBRs with target wavelengths above 480nm. The 460nm DBRs were not included due to the limited ellipsometry range, as mentioned in section 4.3. The layers were then averaged to determine the average refractive indices for all GaN layers and all AlN layers for each DBR. The results were plotted against the TMIn/TMAl ratio in figures 5.3.1 and 5.3.2 for GaN and AlN, respectively, along with second order polynomial fits of the data. A blue line indicates the nominal or expected refractive index.

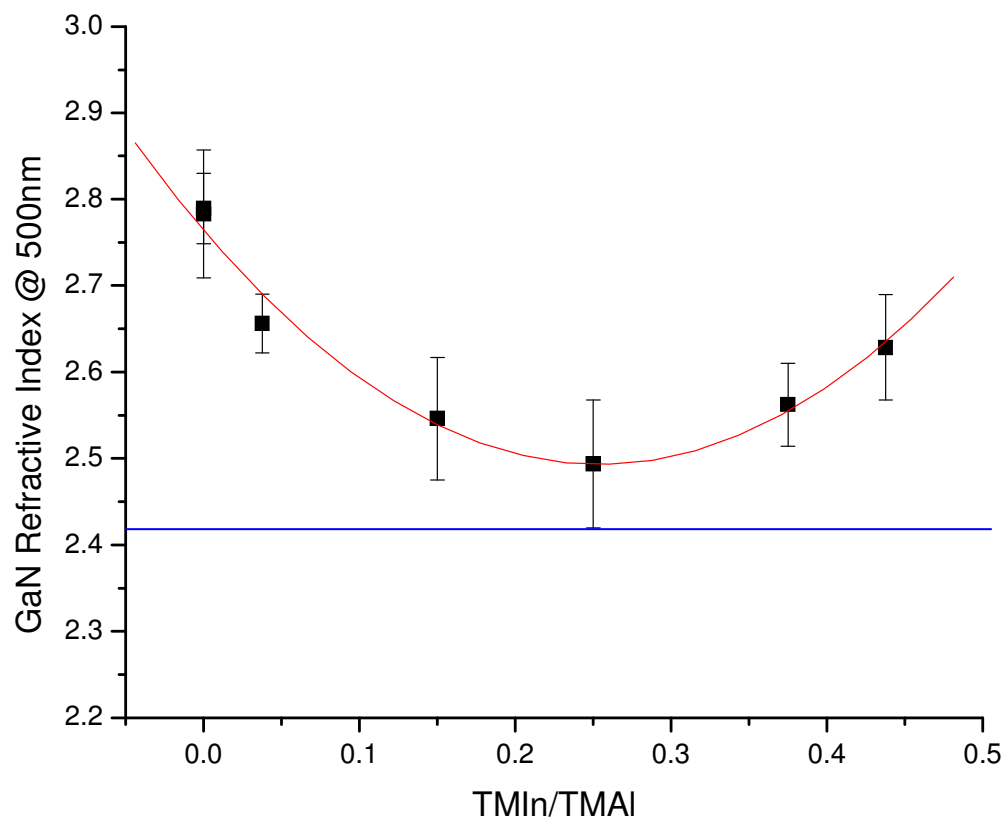

Figure 5.3.1 Average GaN Refractive Index at 500nm for 6-Period DBRs 


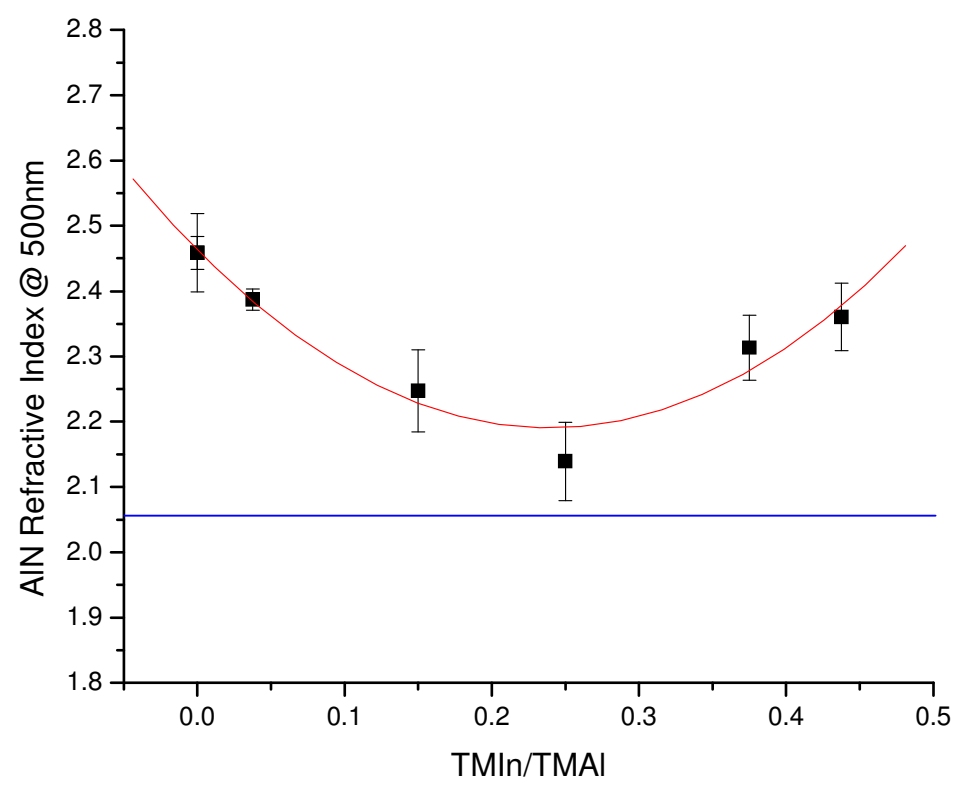

Figure 5.3.2 Average AlN Refractive Index at 500nm for 6-Period DBRs

The apparent refractive indices for both materials were found to vary with the ratio of TMIn/TMAl flow used during the AlN layer growths in a manner similar to that of the domain density. This is an indication that the apparent refractive indices are dependant on the domain density and that increased strain and cracking in the DBRs may be the cause for these higher refractive indices. To determine the dependence of refractive index on domain density, the apparent refractive index for the GaN and AlN layers at 500nm is plotted against domain density in figures 5.3.3 (GaN) and 5.3.4 (AlN). 


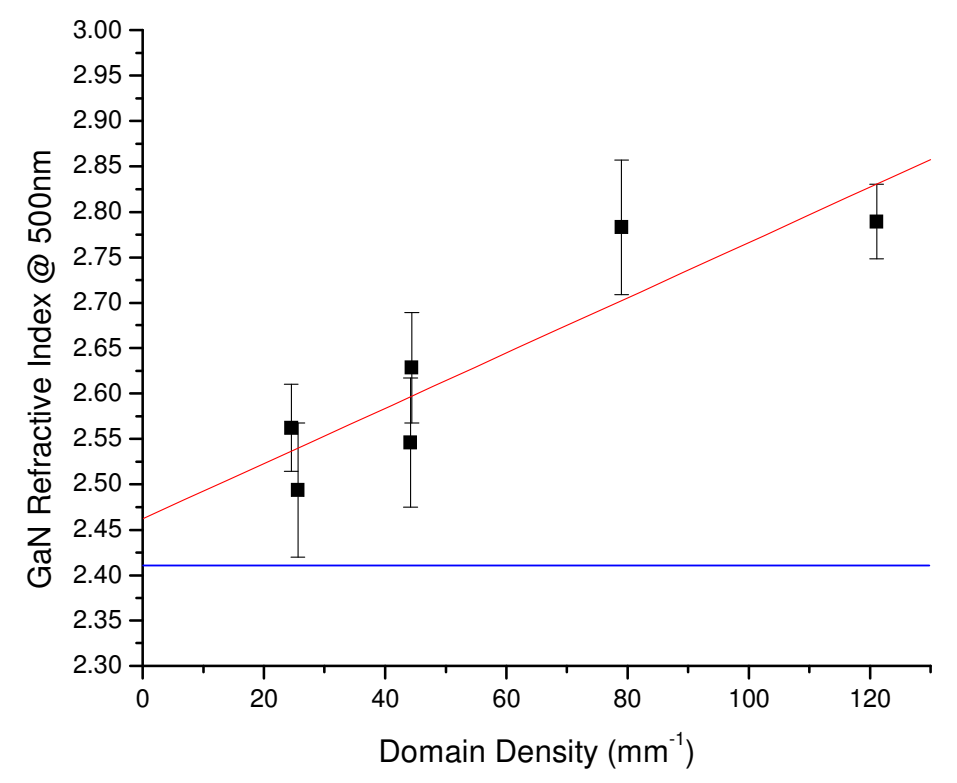

Figure 5.3.3 GaN Refractive Index at 500nm vs. Domain Density

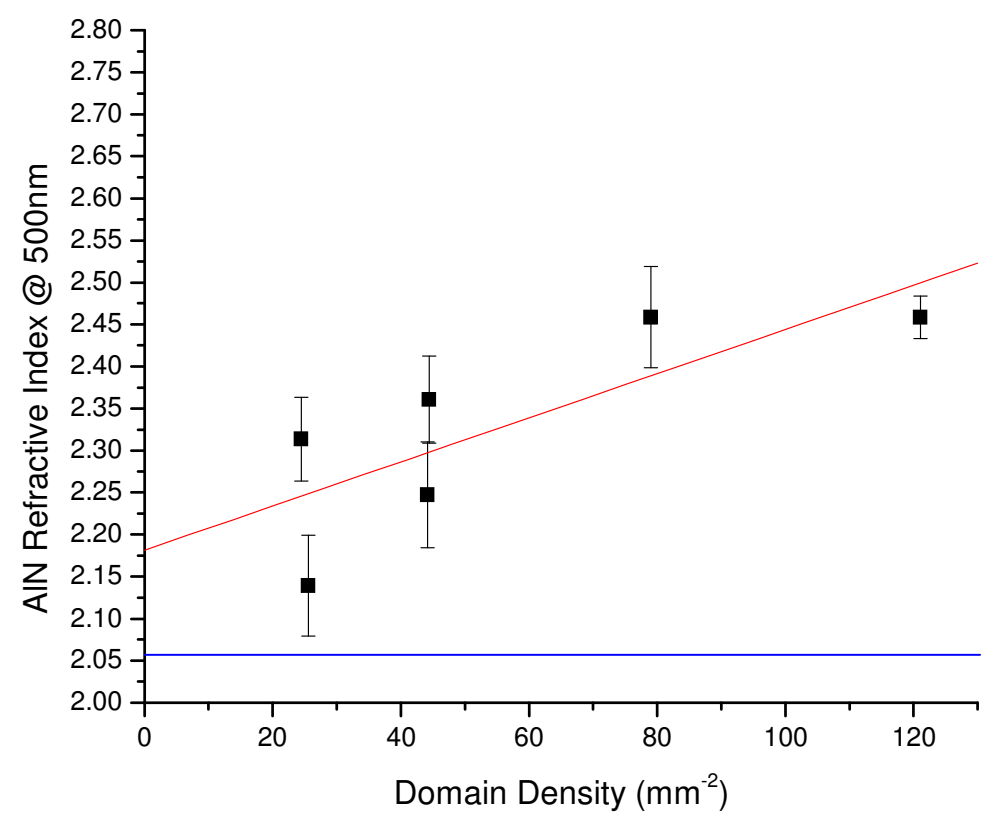

Figure 5.3.4 AlN Refractive Index at 500nm vs. Domain Density 
The apparent refractive index for both GaN and AlN appear to increase linearly with domain density. It is thought that the indices also increase linearly with increased tensile strain as this agrees with what is suggested by literature, but has not been verified [39]. Linear fits were done for both the GaN and AlN data and the fit was extrapolated to find the theoretical values at a domain density of zero. The values found were 2.46 for $\mathrm{GaN}$ and 2.17 for AlN. For GaN, these values were only .04 or $1.65 \%$ off of the index value found for single films. For AlN, they were .1 or $4.8 \%$ off. These values are very close to expected values for good material, especially for $\mathrm{GaN}$, and suggest that a linear relationship between apparent refractive index and domain density may be plausible.

Similar tests were done for three one-period DBRs, one two-period DBR, and one four-period DBR with the results shown in figure 5.3.5. The apparent refractive indices for the DBRs with fewer periods are lower and closer to those found for single films. They may vary in a manner similar to the 6 period DBRs; however, we do not presently have domain density data for these samples, so their dependence on domain density can not yet be verified. Literature suggests less to no cracking in found in DBRs with fewer than 5 periods, so the data would agree with this [43].

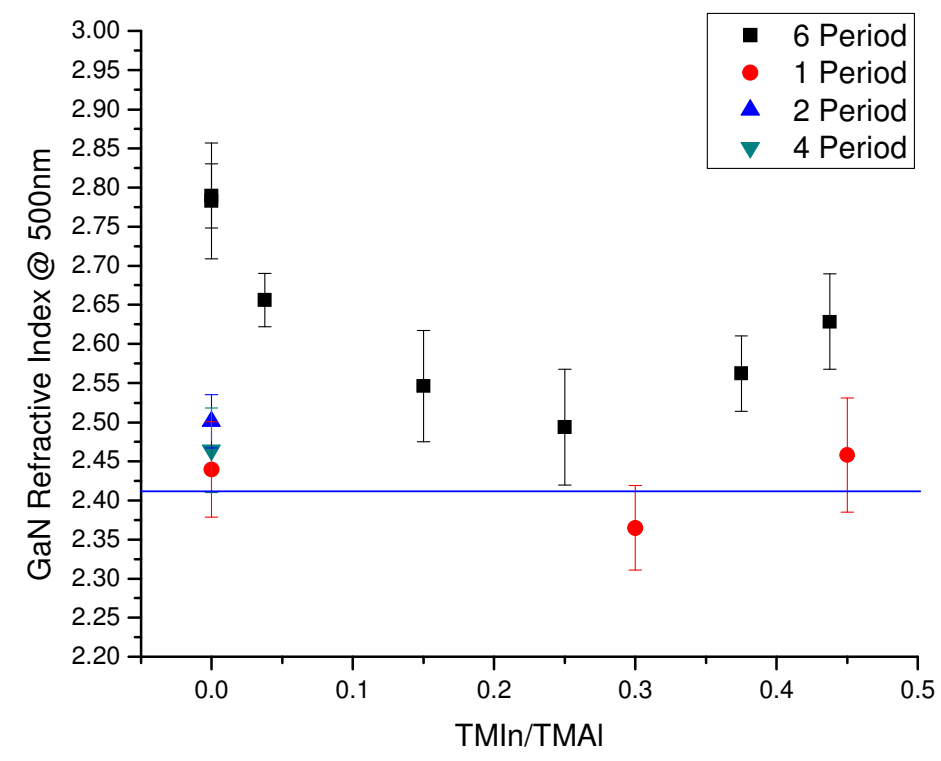

Figure 5.3.5 Average GaN Refractive Index at 500nm for AlN/GaN DBRs 
The reason for these increased apparent refractive indices in the six-period DBRs is believed to be a result of degraded material quality due to strain and the resulting cracking. The refractive indices may be consistent with single films where the material quality is high; however, they are greatly altered by losses due to scattering and diffraction in the poorer, cracked areas [43]. This leads to apparent refractive indices for the overall film that are much higher than expected. It is believed that these increased apparent refractive indices are a result of an increase in domain density and causes decreased reflectivity.

Figures 5.3.6 through 5.3.8 show the reflectivity of DBRs with varying domain densities due the TMIn/TMAl flow ratio used during growth. The samples with higher domain densities have lower reflectivity than ones with lower domain densities. This is thought to be due to losses associated with the poorer material and the resulting increase in refractive indices. Figure 5.3.8 also includes the apparent refractive indices for the DBRs shown. It can be seen that the decreased reflectivity is associated with both increased domain density and increased apparent refractive indices.

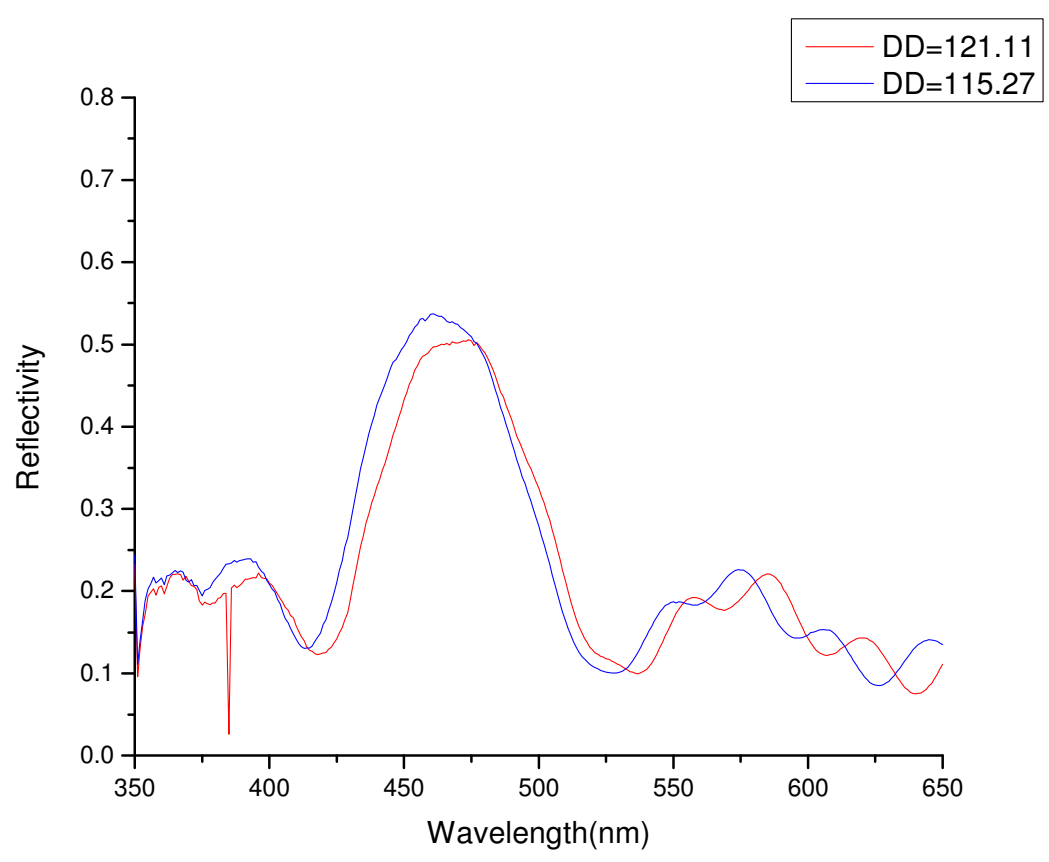

Figure 5.3.6 Reflectivity of 460nm DBRs with Varying Domain Densities 


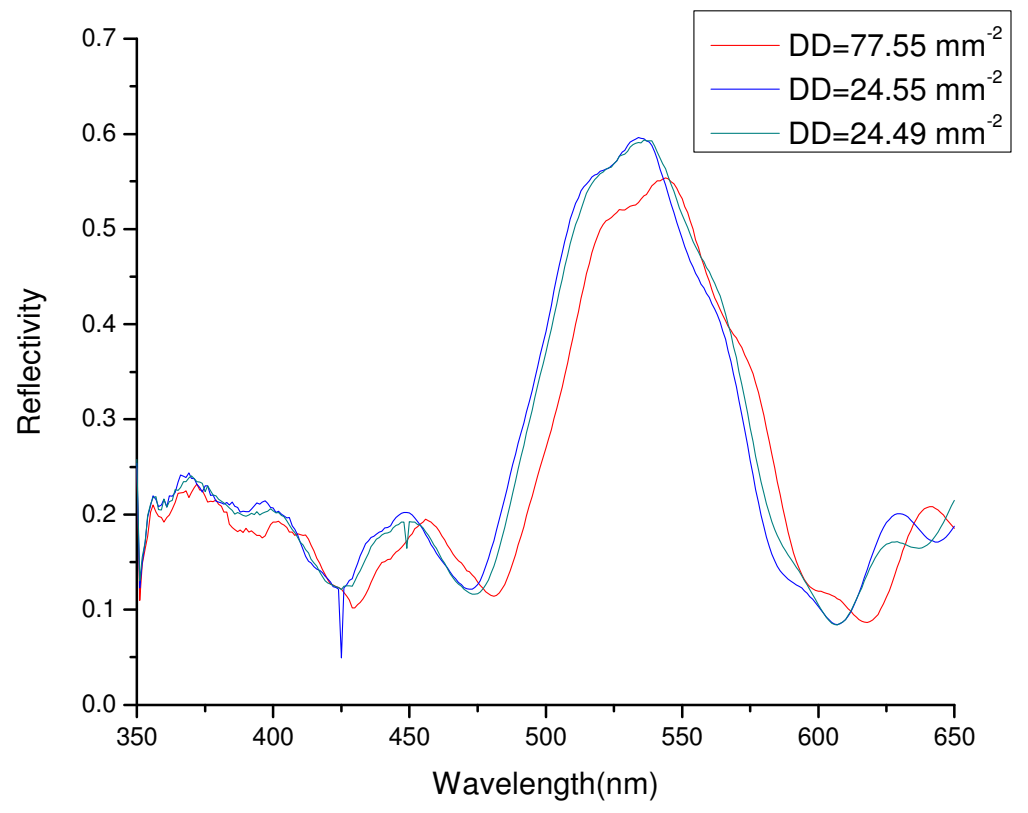

Figure 5.3.7 Reflectivity of 540nm DBRs with Varying Domain Densities

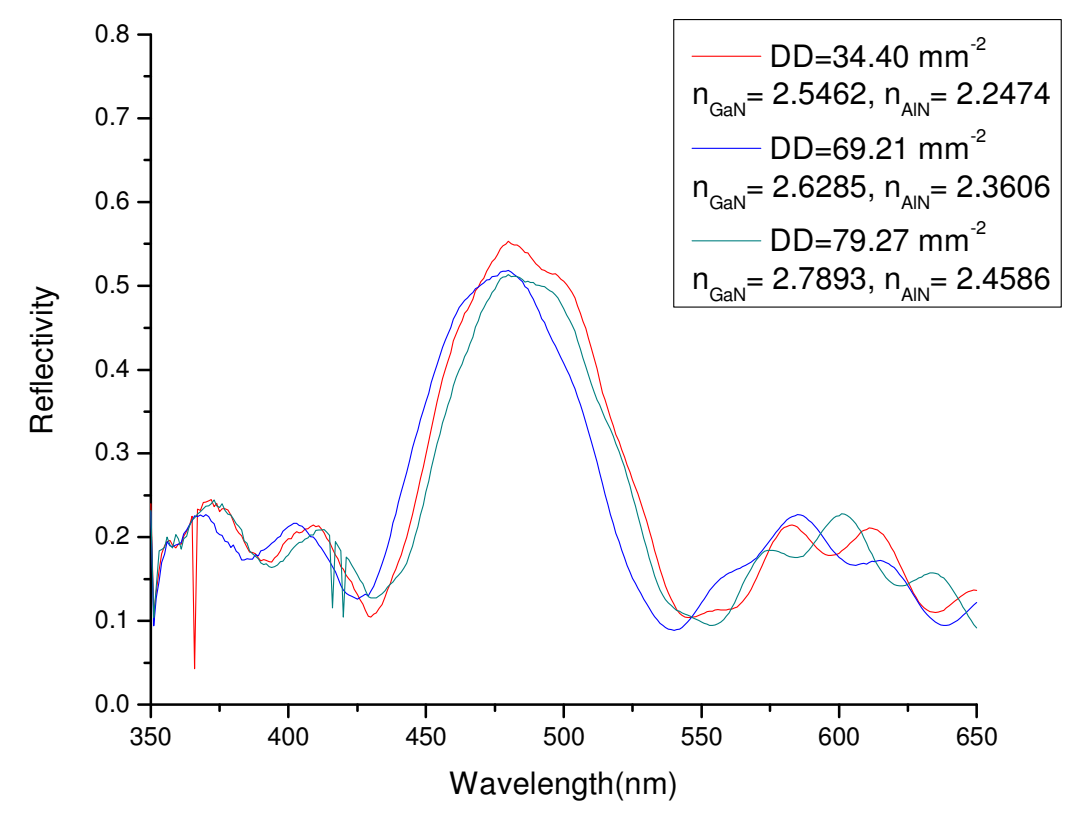

Figure 5.3.8 Reflectivity of 486nm DBRs with Varying Domain Densities 
Figure 5.3.9 shows the peak reflectivity divided by the theoretical reflectivity for these DBRs plotted against the domain density. The reflectivity appears to decrease linearly as domain density increases, but is different for each wavelength due to the GaN and AlN refractive indices varying with wavelength.

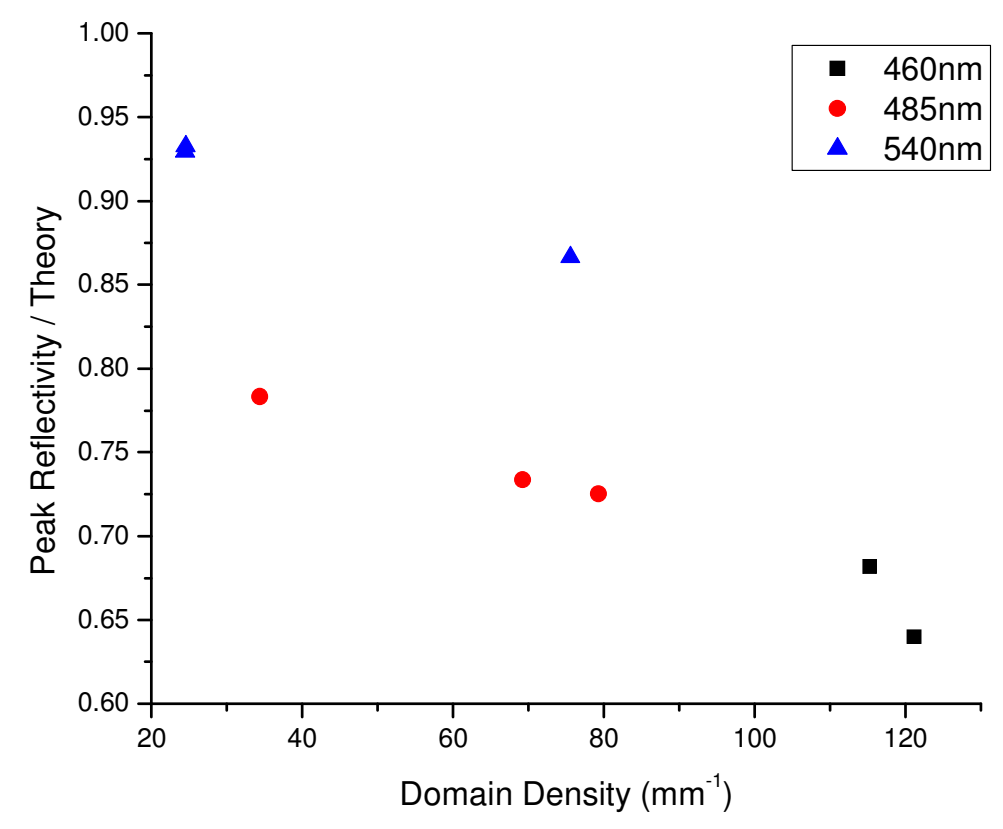

Figure 5.3.9 Peak Reflectivity vs. Domain Density for DBRs

\subsection{Conclusion}

Increased apparent refractive indices were found for both AlN and GaN layers in AlN/GaN DBRs. These increased indices were attributed to tensile strain in the AlN layers as well as cracking as a result of this strain. Successful attempts were made by others in this group to reduce this domain density by adjusting the TMIn/TMAl flow ratio used during growth of AlN layers. It is believed that the Indium has the ability to reduce and absorb tensile strain in the AlN layer by occupying defect states [37, 44]. This reduction in strain directly leads to the reduction in cracking and domain density. 
Apparent refractive indices of both $\mathrm{AlN}$ and GaN were found to be dependant on the TMIn/TMAl flow ratios used during growth. In addition, the apparent refractive indices were found to be linearly dependant on the domain density of samples. By lowering the domain density of samples, the refractive indices of both AlN and GaN layers were able to be brought closer to their nominal values. These increased indices were not as prominent for DBRs with only one, two, or four bilayers, suggesting that the strain and resulting cracking becomes more prominent as the number of layers is increased. In addition, the reflectivity of DBRs was found to be dependant on the domain density and apparent refractive indices of the $\mathrm{AlN}$ and GaN layers. Decreased reflectivity resulted from increased domain densities and apparent refractive indices. Overall, reducing strain and cracking in $\mathrm{AlN} / \mathrm{GaN}$ DBR structures was found to lower the apparent refractive indices and increase overall reflectivity. 


\section{Chapter 6 Conclusions and Future Work}

In this work, III - $\mathrm{N}$ films were characterized in order to develop and improve AlN/GaN Distributed Bragg Reflectors. Optical characterization tools like Variable Angle Spectroscopic Ellipsometry and spectroscopy were used to determine thin film properties such as thickness, refractive index, and bandgap. These properties were used as a baseline to understand and improve material growths in order to expand into more complex structure growths.

AlN/GaN DBRs were grown and characterized. Ellipsometry and spectroscopy were used to determine DBR reflectivity. Ellipsometry was further used to determine thickness and apparent refractive indices of individual layers in DBR structures. The resulting structures were simulated and compared to theoretical values. Simulations were performed using the Transmission Matrix Method, via MATLAB, and the MIT Electromagnetic Equation Propagation software. These simulation methods were able to recreate both the theoretical reflectivity expected and the reflectivity of the actual structures for which thicknesses and apparent refractive indices were determined using ellipsometry. Simulations were also performed to predict and characterize the effects of diffusion between DBR layers as well as the addition of layers like interlayers and buffer layers.

AlN/GaN DBRs were found to have lower reflectivity and higher apparent refractive indices than those predicted by values found from literature as well as those found for single thin films grown under identical growth conditions. The increased apparent refractive indices were attributed to cracking in the samples resulting from tensile strain in the AlN layers caused by lattice mismatch Successful work was done by others to reduce this strain and cracking by adjusting the Indium as a surfactant during AlN layer growth [37]. DBRs with less cracking were found to have apparent refractive indices closer to expected values. These DBRs also exhibited higher reflectivity than samples with more elevated refractive indices. It was concluded that reducing strain in AIN layers led directly to a reduction in cracking and apparent refractive indices; which, in turn, led to an increase in the overall reflectivity of DBRs. 
Additional work does need to be done to more fully understand these structures. The amount of strain in the films needs to be directly quantified to determine the direct effect of strain on apparent refractive indices and reflectivity. The exact mechanism for strain relief also needs to be explored. Improvement can also be made to further reduce the domain density of samples so that the apparent refractive indices further approach those found for single films and the overall reflectivity can be further increased. DBRs with more bilayers need to be grown and characterized to determine if strain can be successfully suppressed for these larger structures as well.

An ability to use these DBR structures as part of actual devices also needs to be demonstrated. The DBRs need to be grown with more bilayers and higher overall reflectivity in order to become useful in practical applications. DBR structures must be demonstrated with sufficient film quality that the growth of additional layers for devices like LEDs and VCSELs is possible. These actual devices must then be grown and tested to determine their capability to be used as practical devices. 


\section{References}

[1] S. C. Jain, M. Willander, J. Narayan, R. Van Overstraeten. J. Appl. Phys 87, 965 (2000).

[2] S. P. Denbaars. Proc. IEEE 85, 1740 (1997).

[3] B. G. Streetman, and S. Banerjee. "Solid State Electronic Devices, Fifth Edition" Prentice Hall, (2000).

[4] R. S. Muller, T. I. Kamins, with M. Chan. "Device Electronics for Integrated Circuits, Third Edition” John Wiley and Sons, (2003).

[5] S. N. Mohammad, A. A. Salvador, and H. Morkoc. Proc. IEEE 83, 1306 (1995).

[6] S. Mokkapati, and C. Jagadish. "III-V compound SC for optoelectronic devices." Elsevier (2009).

[7] L. Turlapati. "Developing UV Photodetector and Ohmic Contact Techniques on GaN.” West Virginia University (2004).

[8] E. Hecht. “Optics, Fourth Edition” Addison Wesley, (2002).

[9] L. E. Rodak, N. J. Berry Ann, K. R. Kasarla, N. Yang, and D. Korakakis. Mater. Res. Symp. Proc. 892 (2006).

[10] A. Yamamoto, T. Tanikawa, B. K. Ghosh, Y. Hamano, A. Hashimoto, and Y. Ito. Phys. Stat. Sol. (C). 0, 2562 (2003).

[11] M. Leszczynski, H. Teisseyre, T. Suski, I Grzegory, M. Bockowskit, J. Jun, S. Porowski, K. Pakula, J. M. Baranowski, C. T. Foxon, and T. S. Cheng. Appl. Phys. Lett. 69, 73 (1996).

[12] S. Ramo, J. R. Whinnery, and T. Van Duzer. "Fields and Waves in Communication Electronics, Third Edition” John Wiley and Sons, (1994).

[13] H. Motschmann, R. Teppner "Ellipsometry in Interface Science" (2001).

[14] J. A. Woollam Co., Inc., "A Short Course in Ellipsometry,” (2001).

[15] D. Goldstein. "Polarized Light, Second Edition” CRC Oress, (2003). 
[16] N. Nakada, M. Nakaji, H. Ishikawa, T. Egawa, M. Umeno, and T. Jimbo. Appl. Phys. Lett. 76, 1804 (2000).

[17] C.J. Chang-Hasnain. IEEE J. on Select Topics in Quantum Electronics. 6:6, 978 (2000).

[18] N. Nakada, H. Ishikawa, T. Egawa, and T. Jimbo. Jpn. J. Appl. Phys. 42. L144 (2003).

[19] E. Alsema, P. Frankl, and K. Kato. "Energy Pay-Back Time of Photovoltaic Energy Systems: Present Status and Prospects" 2nd World Conference on Photovoltaic Solar Energy Conversion. Vienna (1998).

[20] R. A. Serway, and R. J. Beichner. "Physics for Scientists and Engineers, Fifth Edition" Thomson Learning, (2000).

[21] T. Yang, S. Goto, M. Kawata, K. Uchida, A. Niwq, and J. Gotoh. Jpn. J. Appl. Phys. 37. L1105 (1998).

[22] “Snell’s law.” Encyclopcedia Britannica. Retrieved June 27, 2009.

[23] H. M. Reese. "Light” Missouri Book Company, (1921).

[24] S. M. Sze and Kwok K. Ng. "Physics of Semiconductor Devices, Third Edition" Cambridge University Press, (2006).

[25] http://www.rpi.edu/ schubert/Educational-resources/Materials-Refractive-index-andextinction-coefficient.pdf

[26] G. Yu, H. Ishikawa, M. Umeno, T. Egawa, J. Watanabe, T. Jimbo, and T. Soga. Appl. Phys. Lett. 72, 2202 (1998).

[27] D. Korakakis, H. M. Ng, M. Misra, W. Grieshaber, and T. D. Moustakas. MRS Internet J. Nitride Semicond. Res. 1, 10 (1996).

[28] D. Brunner, H. Angerer, E. Bustarret, F. Freudenberg, R. Höpler, R. Dimitrov, O. Ambacher, and M. Stutzmann. J. Appl. Phys 82, 5090 (1997).

[29] H. M. Ng, D. Doppalapudi, E. Iliopoulos, and T. D. Moustakas. Appl. Phys. Lett. 74, 1036 (1999).

[30] H. A. Macleod, “Thin Film Optical Filters, Second Edition,” McGraw-Hill, (1989).

[31] MEEP Manual (]http://ab-initio.mit.edu/wiki/index.php/Meep).

[32] C. W. Wilmsen, H. Temkin, and L. A. Coldren. "Vertical-Cavity Surface-Emitting Lasers," Cambridge University Press, (1999). 
[33] K. E. Waldrip, J. Han, J. J. Figiel, H. Zhou, E. Makarona, and A. V. Nurmikko, Appl. Phys. Lett. 78, 3205 (2001).

[34] J.-F. Carlin, C. Zellweger, J. Dorsaz, S. Nicolay, G. Christmann, E. Feltin, R. Butté, and N. Grandiean. Phys. Stat. Sol. (b). 242, 2326 (2005).

[35] F. Natali, D. Byrne, A. Dussaigne, N. Grandjean, J. Massies, and B. Damilano, Appl. Phys. Lett. 82, 499 (2003).

[36] J.-F. Carlin, J. Dorsaz, E. Feltin, R. Butte, N. Grandjean, M. Ilegems, and M. Laugt, Appl. Phys. Lett. 86, 031107 (2005).

[37] L. E. Rodak, and D. Korakakis. Mater. Res. Symp. Proc. 1108 (2009).

[38] H. M. Ng, T. D. Moustakas, and S. N. G. Chu, Appl. Phys. Lett. 76, 2818 (2000).

[39] S. Pezzagna, J. Brault, M. Leroux, J. Massies, and M. de Micheli, J. Appl. Phys. 103, 123112 (2008).

[40] S. C. Jones, M. C. Robinson, and Y. M. Gupta, J. Appl. Phys. 93, 1023 (2003).

[41] S. Hearne, E. Chason, J. Han, J. A. Floro, J. Figiel, J. Hunter, H. Amano, and I. S. T. Tsong. Appl. Phys. Lett. 74, 356 (1999).

[42] A. Bhattacharyya, S. Iyer, E. Iliopoulos, A. V. Sampath, J. Cabalu, and T. D. Moustakas. J. Vac. Sci. Technol. B 20, 1229 (2002).

[43] G. S. Huang, T. C. Lu, H. H. Yao, H. C. Kuo, S. C. Wang, C. Lin, and L. Chang. Appl. Phys. Lett. 88, 061904 (2006).

[44] S. Yamaguchi, M. Kariya, T. Kashima, S. Nitta, M. Kosaki, and Y. Tukawa. Phys. Rev. B. 64, 035318 (2001). 


\section{Appendix A: List of Acronyms}

$\begin{array}{ll}\text { AlN } & : \text { Aluminum Nitride } \\ \text { AlGaN } & : \text { Aluminum Gallium Nitride } \\ \text { AlGaInN } & : \text { Aluminum Gallium Indium Nitride } \\ \text { AlInN } & : \text { Aluminum Indium Nitride } \\ \text { DBR } & : \text { Distributed Bragg Reflector } \\ \text { GaN } & : \text { Gallium Nitride } \\ \text { HVPE } & : \text { Hydride Vapor Phase Epitaxy } \\ \text { III - N } & : \text { Group III - Nitride Semiconductors } \\ \text { InGaN } & : \text { Indium Gallium Nitride } \\ \text { InN } & : \text { Indium Nitride } \\ \text { LED } & : \text { Light Emitting Diode } \\ \text { MBE } & : \text { Molecular Beam Epitaxy } \\ \text { MEEP } & : \text { MIT Electromagnetic Equation Propogation } \\ \text { MOVPE } & : \text { Metal Organic Vapor Phase Epitaxy } \\ \text { MQW } & : \text { Multiple Quantum Well } \\ \text { MSE } & : \text { Mean Squared Error } \\ \text { NH3 } & : \text { Ammonia } \\ \text { TMAl } & : \text { Trimethylaluminum } \\ \text { TMGa } & : \text { Trimethylgallium } \\ \text { TMIn } & : \text { Trimethylindium } \\ \text { TMM } & : \text { Transmission Matrix Method } \\ \text { VASE } & : \text { Variable Angle Spectroscopic Ellipsometry (Ellipsometer) } \\ \text { VCSEL } & : \text { Vertical Cavity Surface Emitting Laser }\end{array}$




\section{Appendix B: MATLAB Code}

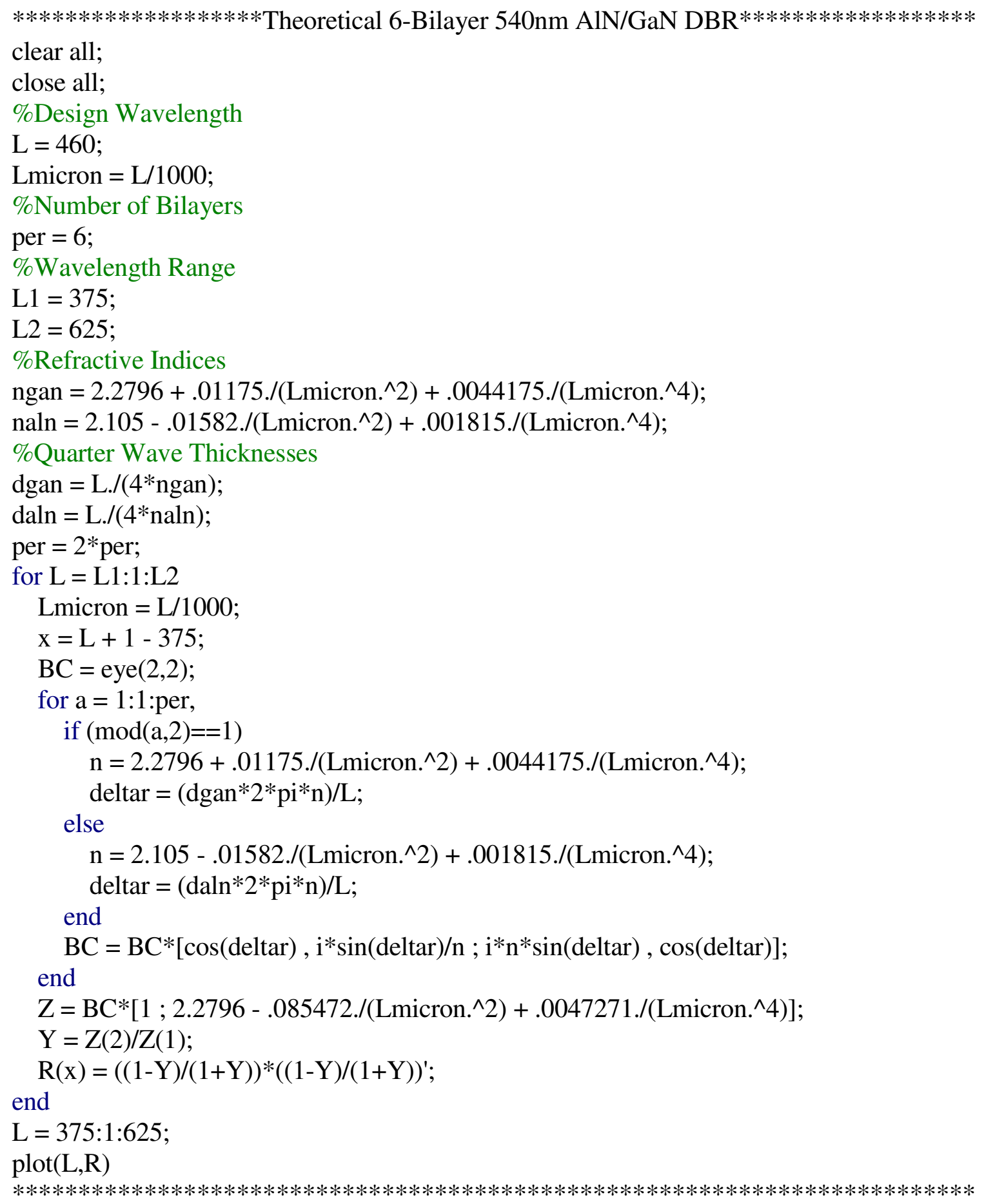




\section{Appendix C: MEEP Code}

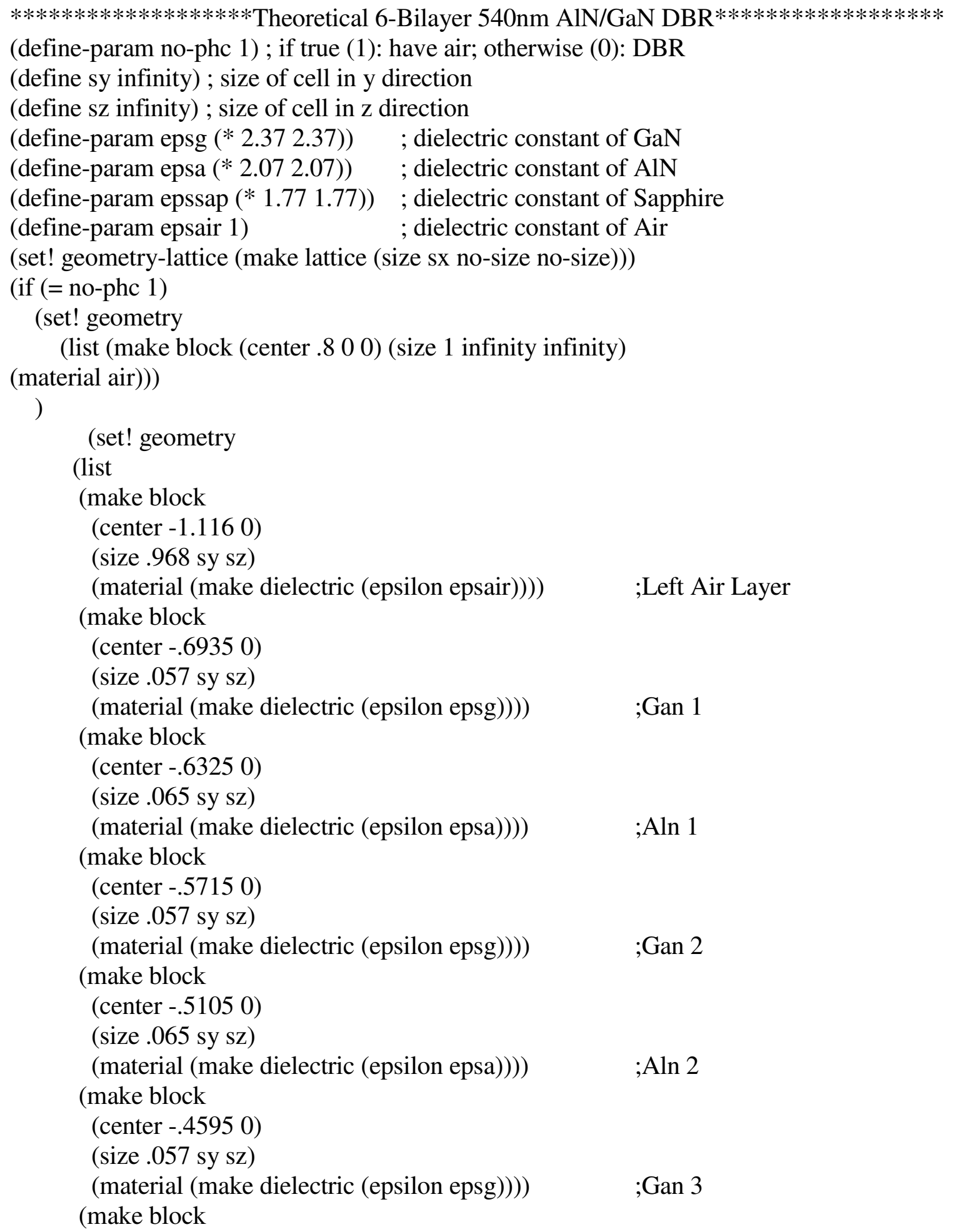




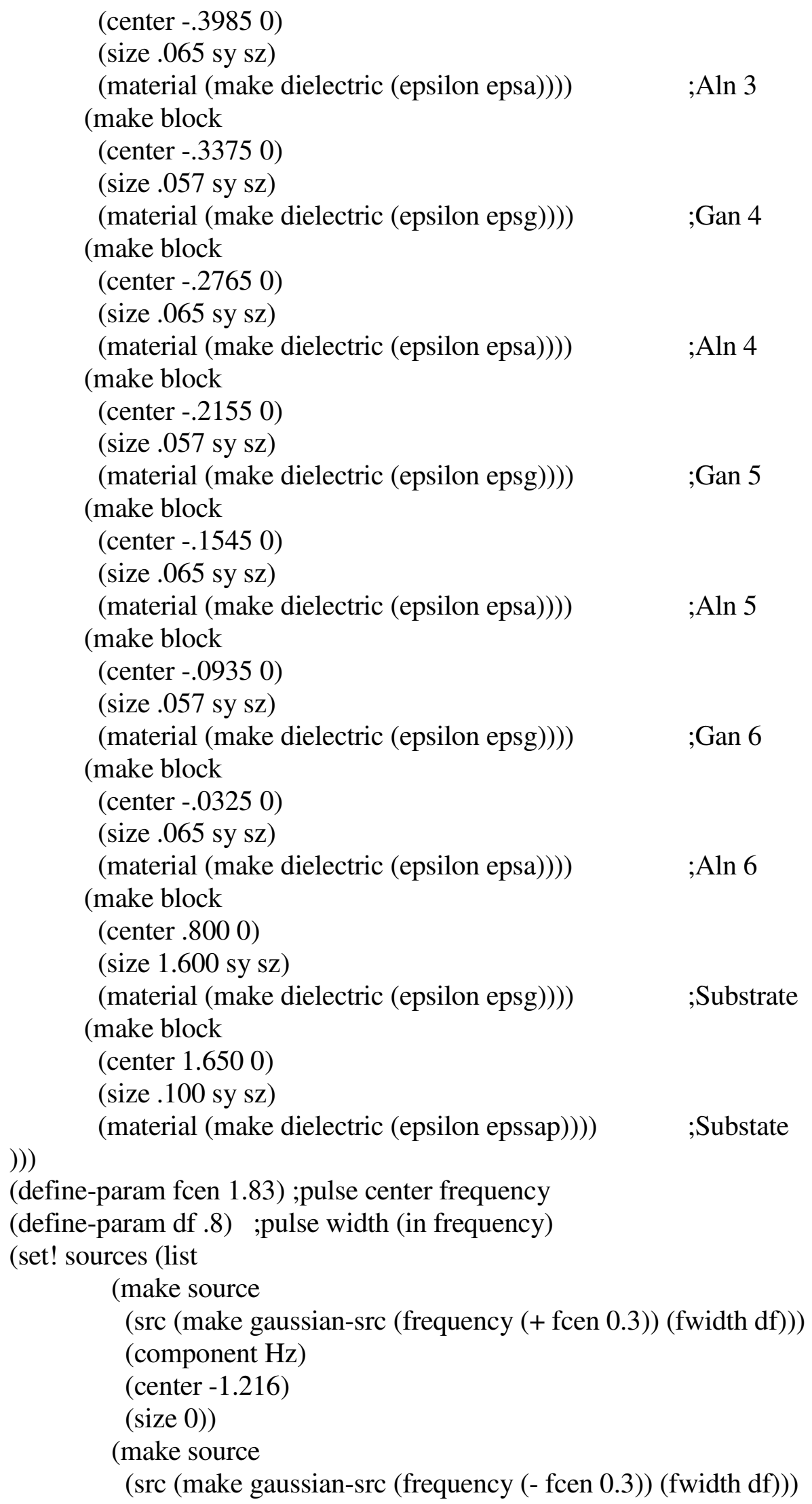




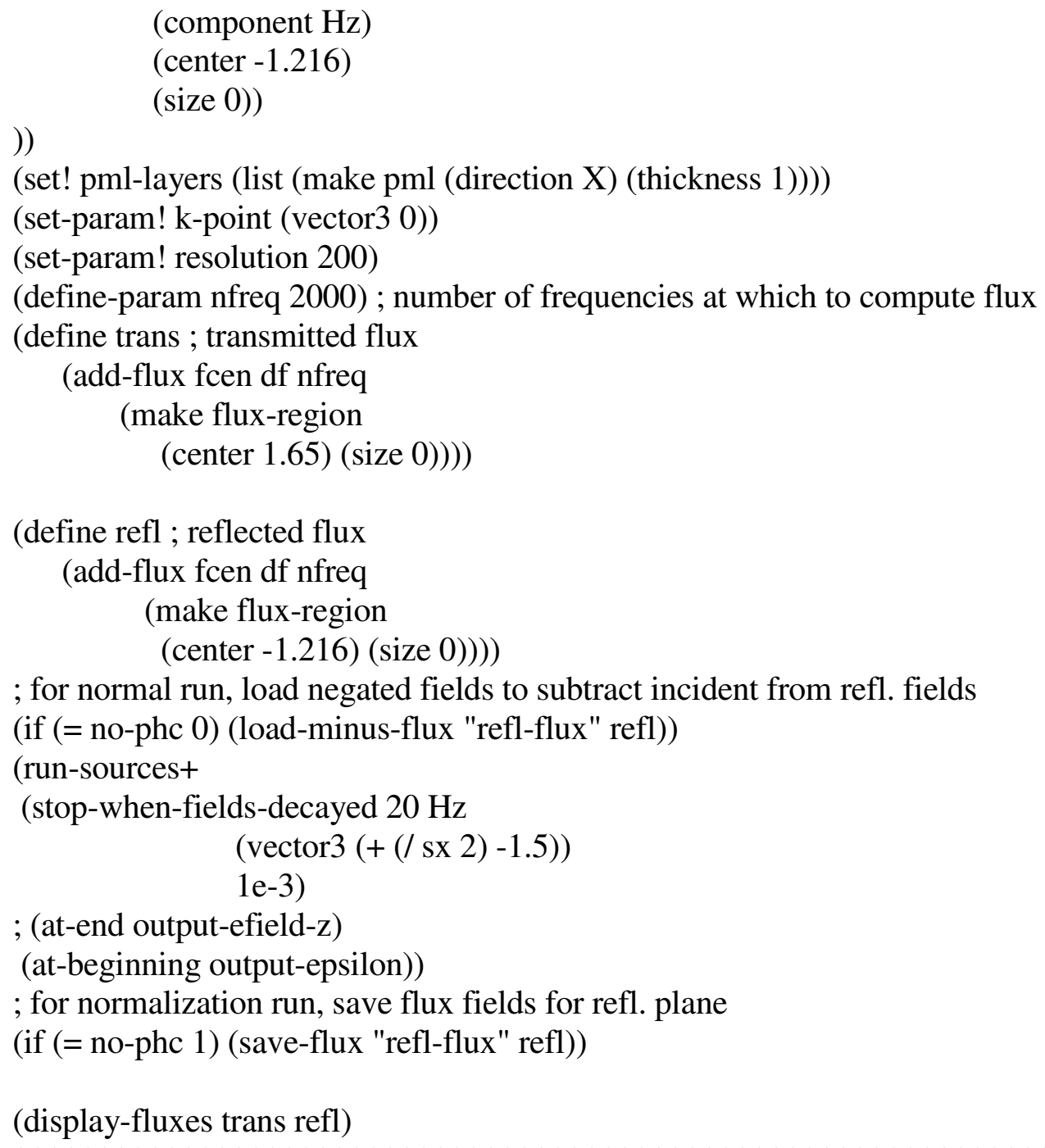




\section{Appendix D: Ellipsometry Protocols and Notes}

\section{Scan Process}

- Turn on power on EC-400 box

- Turn on power on M-2000U box

- Press Lamp Ignition on M-2000U box

- Open V.A.S.E (Wvase32) software on the computer desktop

- On top right of screen, click 'Window,' then 'Hardware' in drop down menu

- Right click in newly opened 'Hardware' window, then select 'Initialize'

- Place sample on Ellipsometer

- Once initialized, right click in 'Hardware' window and select 'Acquire Data' > 'Align Sample'

- Turn knobs on ellipsometer until the ' + ' is aligned in the center

- In hardware window, select 'Acquire Data' > 'Spectroscopic Scan'

- Select scan angles (usually $55^{\circ}$ to $65^{\circ}$ by $5^{\circ}$ )

- Click ok to scan

- Save experimental data

\section{Model Creation}

- Leave scanned data from the 'Scan Process' section open or open previously saved data in the 'Experimental Data' window

- In the 'Model' window, load or create an optical model of what the sample is expected to be (Examples are shown in the sample models section)

- Input expected thickness for each layer

- Input expected refractive indices for each layer based on literature or previous scans (Literature Cauchy values for AlN and GaN found in this thesis in section 3.2.2)

- In the "Model" window, blue lettering indicates variable values while black indicates fixed values

- Choose to have thicknesses and/or refractive indices in each layer to be fixed if desired, though this well inhibit the overall accuracy of the model

\section{Fitting and Output}

- In 'Fit' window, select 'Normal Fits' > 'Grid Global Fit' or other fitting method

- Continue selecting this until notified "Change in MSE is less than limit" or until satisfied with fitting results

- Judge the model on the MSE as well as the graphs in the 'Graph' window.

- Especially for single thin films: if the peaks do not line up in the 'Graph' window, you may need to adjust the starting thicknesses iteratively in the model and start the fits over 
- The MSE varies largely, but should be definitely be less than 80 for single films and less than 30 for 6 period DBRs to be considered a decent fit

- Quality of fit should be judged on the 'Graph' window results, the MSE, and comparing to model to expectations and values determined with other characterization methods such as spectroscopy and SEM

- Read output values listed in the 'Fit' window or click on layers in 'Model" window to determine output values

\section{Notes}

- Cauchy models of refractive indices are only effective for energies below the bandgap, above which the extinction coefficient must be taken into account

- Well fitting, completed models are accurate representations of the interaction of light with the optical system, but may not always represent the actual structure and may represent a structure with equivalent optical impedance. Comparison of results with other characterization methods is critical to verifying ellipsometry results. This especially critical for structures with many layers like DBRs

- Reflectivity for DBRs should be recreated using simulation methods discussed in this thesis and compared to the actual reflectivity determined using spectroscopy in order ensure that the values determined are successful in representing the optical system

- Ellipsometry was found to be ineffective in modeling DBRs with target wavelengths lower than $480 \mathrm{~nm}$, which was attributed to the limited range of the light source and detector

- It is often beneficial to fit data with both fixed and variable refractive indices for additional comparison data to determine the merit of fits

\section{Example Models}

\begin{tabular}{|llr|}
\hline 2 & srough & $1.000 \mathrm{~nm}$ \\
\hline 1 & cauchy gan & $1600.000 \mathrm{~nm}$ \\
\hline 0 & al203 & $1 \mathrm{~mm}$
\end{tabular}

Single Gallium Nitride Thin Film on Sapphire with a Surface Roughness Component 


\begin{tabular}{|llr|}
\hline 3 & srough & $1.000 \mathrm{~nm}$ \\
\hline 2 & cauchy aln & $300.000 \mathrm{~nm}$ \\
\hline 1 & cauchy gan & $1000.000 \mathrm{~nm}$ \\
\hline 0 & al203 & $1 \mathrm{~mm}$
\end{tabular}

Aluminum Nitride on Gallium Nitride on Sapphire Sample with a Surface Roughness Component

\begin{tabular}{|llr|}
\hline 13 & cauchygan & $56.918 \mathrm{~nm}$ \\
\hline 12 & cauchyaln & $65.151 \mathrm{~nm}$ \\
\hline 11 & cauchygan & $56.918 \mathrm{~nm}$ \\
\hline 10 & cauchyaln & $65.151 \mathrm{~nm}$ \\
\hline 9 & cauchygan & $56.918 \mathrm{~nm}$ \\
\hline 8 & cauchyaln & $65.151 \mathrm{~nm}$ \\
\hline 7 & cauchygan & $56.918 \mathrm{~nm}$ \\
\hline 6 & cauchyaln & $65.151 \mathrm{~nm}$ \\
\hline 5 & cauchygan & $56.918 \mathrm{~nm}$ \\
\hline 4 & cauchyaln & $65.151 \mathrm{~nm}$ \\
\hline 3 & cauchygan & $56.918 \mathrm{~nm}$ \\
\hline 2 & cauchyaln & $65.151 \mathrm{~nm}$ \\
\hline 1 & cauchygan & $1600.000 \mathrm{~nm}$ \\
\hline 0 & al2o3 & $1 \mathrm{~mm}$
\end{tabular}

6 Period AlN/GaN Distributed Bragg Reflector on GaN on Sapphire with Each DBR Layer Independent 


$\left.\begin{array}{|llr|}\hline 3 & \text { cauchygan } & 56.918 \mathrm{~nm} \\ \hline 2 & \text { cauchyaln } & 65.152 \mathrm{~nm} \\ \hline 1 & \text { cauchygan } & 1600.000 \mathrm{~nm} \\ \hline 0 & \text { al203 } & 1 \mathrm{~mm}\end{array}\right] 6$

6 Period AlN/GaN Distributed Bragg Reflector on GaN on Sapphire Employing a Superlattice

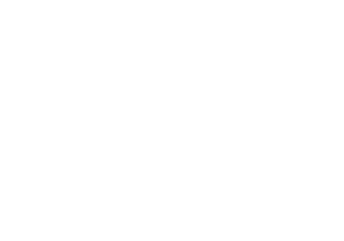

